doi:10.1017/S1474748021000451 (C) The Author(s), 2021. Published by Cambridge University Press. This is an Open Access article, distributed under the terms of the Creative Commons Attribution licence (https://creativecommons.org/licenses/by/4.0), which permits unrestricted re-use, distribution and reproduction, provided the original article is properly cited.

\title{
ON THE CHOW THEORY OF PROJECTIVIZATIONS
}

\author{
QINGYUAN JIANG \\ School of Mathematics, University of Edinburgh, JCMB, Peter Guthrie Tait Road, \\ Edinburgh EH9 3FD, UK \\ (Qingyuan.Jiang@ed.ac.uk)
}

(Received 20 August 2020; revised 30 August 2021; accepted 31 August 2021; first published online 11 November 2021)

\begin{abstract}
In this paper, we prove a decomposition result for the Chow groups of projectivizations of coherent sheaves of homological dimension $\leq 1$. In this process, we establish the decomposition of Chow groups for the cases of the Cayley trick and standard flips. Moreover, we apply these results to study the Chow groups of symmetric powers of curves, nested Hilbert schemes of surfaces, and the varieties resolving Voisin maps for cubic fourfolds.
\end{abstract}

Key words and phrases: Chow groups, motives, projectivizations, flips, curves, nested Hilbert schemes 2020 Mathematics Subject Classification: Primary 14C15, 14C25, 14E05, 14H51, 14D06

\section{Introduction}

Let $X$ be a Cohen-Macaulay scheme of pure dimension and $\mathscr{G}$ a coherent sheaf on $X$ of rank $r$ and homological dimension $\leq 1$ - that is, locally over $X$, there is a two-step resolution $0 \rightarrow \mathscr{F} \rightarrow \mathscr{E} \rightarrow \mathscr{G} \rightarrow 0$, where $\mathscr{F}$ and $\mathscr{E}$ are finite locally free sheaves. (If $X$ is regular, this condition on $\mathscr{G}$ is equivalent to $\mathscr{E} x t_{X}^{i}\left(\mathscr{G}, \mathscr{O}_{X}\right)=0$ for all $i \geq 2$.) The projectivization $\pi: \mathbb{P}(\mathscr{G}):=\operatorname{Proj}_{X} \operatorname{Sym}_{\mathscr{O}_{X}}^{\bullet} \mathscr{G} \rightarrow X$ of $\mathscr{G}$ is generically a projective bundle with fiber $\mathbb{P}^{r-1}$; however, the dimension of the fiber of $\pi$ jumps along the degeneracy loci (see $\S 2.1$ ) of $\mathscr{G}$.

The derived category of $\mathbb{P}(\mathscr{G})$ was studied in [25], where we proved (under certain regularity and dimension conditions) that there is a semiorthogonal decomposition

$$
\mathrm{D}_{\mathrm{coh}}^{\mathrm{b}}(\mathbb{P}(\mathscr{G}))=\left\langle\mathrm{D}_{\mathrm{coh}}^{\mathrm{b}}\left(\mathbb{P}\left(\mathscr{E} x t^{1}\left(\mathscr{G}, \mathscr{O}_{X}\right)\right)\right), \mathrm{D}_{\mathrm{coh}}^{\mathrm{b}}(X) \otimes \mathscr{O}(1), \ldots, \mathrm{D}_{\mathrm{coh}}^{\mathrm{b}}(X) \otimes \mathscr{O}(r)\right\rangle .
$$

(For a space $Y, \mathrm{D}_{\text {coh }}^{\mathrm{b}}(Y)$ stands for its bounded derived category of coherent sheaves.) The theorem states that the (right) orthogonal of the 'projective bundle part' of $\mathrm{D}_{\mathrm{coh}}^{\mathrm{b}}(\mathbb{P}(\mathscr{G}))$ is given by the derived category of another projectivization $\mathbb{P}\left(\mathscr{E} x t^{1}\left(\mathscr{G}_{,} \mathscr{O}_{X}\right)\right)$, which is a Springer-type partial desingularization of the singular locus of $\mathscr{G}$ (see [25] for more details). 
In this paper, we establish the Chow-theoretic version of this formula:

Theorem (see Theorem 4.1). Let $X$ and $\mathscr{G}$ be as before. Assume either

(A) $\mathbb{P}(\mathscr{G})$ and $\mathbb{P}\left(\mathscr{E} x t^{1}\left(\mathscr{G}, \mathscr{O}_{X}\right)\right)$ are nonsingular and quasi-projective, and the degeneracy loci of $\mathscr{G}$ satisfy a weak dimension condition (4.1); or

(B) all degeneracy loci of $\mathscr{G}$ (either are empty or) have expected dimensions.

Then for each $k \geq 0$, there is an isomorphism of integral Chow groups:

$$
\mathrm{CH}_{k}(\mathbb{P}(\mathscr{G})) \simeq \mathrm{CH}_{k-r}\left(\mathbb{P}\left(\mathscr{E} x t^{1}\left(\mathscr{G}, \mathscr{O}_{X}\right)\right)\right) \oplus \bigoplus_{i=0}^{r-1} \mathrm{CH}_{k-(r-1)+i}(X) .
$$

Since the isomorphism of the theorem commutes with the product with another space, by Manin's identity principle, if $\mathbb{P}(\mathscr{G}), \mathbb{P}\left(\mathscr{E} x t^{1}\left(\mathscr{G}, \mathscr{O}_{X}\right)\right)$ and $X$ are smooth and projective over the ground field $\mathbb{k}$, then there is an isomorphism of integral (pure effective) Chow motives:

$$
\mathfrak{h}(\mathbb{P}(\mathscr{G})) \simeq \mathfrak{h}\left(\mathbb{P}\left(\mathscr{E} x t^{1}\left(\mathscr{G}, \mathscr{O}_{X}\right)\right)\right)(r) \oplus \bigoplus_{i=0}^{r-1} \mathfrak{h}(X)(i)
$$

(see Corollary 4.3). Note that this result compares nicely with Vial's work [52] on $\mathbb{P}^{r-1}$-fibrations; in our case, $\mathbb{P}(\mathscr{G})$ is a generic $\mathbb{P}^{r-1}$-fibration. Taking a cohomological realization - for example, the Betti cohomology if $\mathbb{k} \subset \mathbb{C}$ - the isomorphism of motives induces an isomorphism of rational Hodge structures:

$$
H^{n}(\mathbb{P}(\mathscr{G}), \mathbb{Q}) \simeq H^{n-2 r}\left(\mathbb{P}\left(\mathscr{E} x t^{1}\left(\mathscr{G}, \mathscr{O}_{X}\right)\right), \mathbb{Q}\right) \oplus \bigoplus_{i=0}^{r-1} H^{n-2 i}(X, \mathbb{Q}), \quad \forall n \geq 0 .
$$

This paper provides two approaches to proving our theorem, one under each of the conditions (A) and (B). The idea behind both approaches is that one could view the projectivization phenomenon as a combination of the Cayley trick and flips.

We study the Chow theory for the Cayley trick in $§ 3.1$ (see Theorem 3.1 and Corollary 3.4) and the Chow theory of standard flips in $\S 3.2$ (see Theorem 3.6 and Corollary 3.10). These results are of independent interest on their own. For example, it follows from Theorem 3.1 and Corollary 3.4 that the Chow group (and., motive) of every complete intersection variety can be split-embedded into that of a Fano variety (see Example 3.5; compare [28]).

The first examples of the theorem are universal Hom spaces (see §4.3.1), flops from Springer-type resolutions of determinantal hypersurfaces (see §4.3.2), and a blowup formula for blowing up along Cohen-Macaulay codimension 2 subschemes (see §4.3.3).

\subsection{Applications}

The following applications parallel the applications of the projectivization formula in the study of derived categories [25].

(1) Symmetric powers of curves (\$5.1). Let $C$ be a smooth projective curve of genus $g \geq 1$ and denote by $C^{(k)}$ the $k$ th symmetric power. For any $0 \leq n \leq g-1$, the 
relationships between the derived category of $C^{(g-1+n)}$ and $C^{(g-1-n)}$ (and also the Jacobian variety $\operatorname{Jac}(C)$ ) was established by Toda [51] using wall crossing of stable pair moduli, and later by [5, 25] using the projectivization formula. The main theorem of this paper implies the corresponding Chow-theoretic version of the formula: for any $k \geq 0$, there is an isomorphism of integral Chow groups

$$
\mathrm{CH}_{k}\left(C^{(g-1+n)}\right) \simeq \mathrm{CH}_{k-n}\left(C^{(g-1-n)}\right) \oplus \bigoplus_{i=0}^{n-1} \mathrm{CH}_{k-(n-1)+i}(\operatorname{Jac}(C)),
$$

and a similar decomposition for integral Chow motives (see Corollary 5.1).

(2) Nested Hilbert schemes of surfaces (\$5.2). Let $S$ be a smooth quasi-projective surface, and denote by $\operatorname{Hilb}_{n}(S)$ the Hilbert scheme of $n$ points on $S$; by convention, $\operatorname{Hilb}_{1}(S)=S, \operatorname{Hilb}_{0}=$ point. Denote $\operatorname{Hilb}_{n, n+1}(S)$ the nested Hilbert scheme. Then the projectivization formula of derived categories [25] can be applied to obtain a semiorthogonal decomposition of $D\left(\operatorname{Hilb}_{n, n+1}(S)\right)$ [5]. In this paper we show that for any $k \geq 0$, there is an isomorphism of integral Chow groups

$$
\begin{aligned}
\mathrm{CH}_{k}\left(\operatorname{Hilb}_{n, n+1}(S)\right) & \simeq \mathrm{CH}_{k-1}\left(\operatorname{Hilb}_{n-1, n}(S)\right) \oplus \mathrm{CH}_{k}\left(\operatorname{Hilb}_{n}(S) \times S\right) \\
& \simeq \bigoplus_{i=0}^{n} \mathrm{CH}_{k-i}\left(\operatorname{Hilb}_{n-i}(S) \times S\right),
\end{aligned}
$$

and a similar decomposition for Chow motives (see Corollary 5.4).

(3) Voisin maps (§5.3). Let $Y$ be a cubic fourfold not containing any plane, let $F(Y)$ be the Fano variety of lines on $Y$, and let $Z(Y)$ be the corresponding LLSvS eightfold [36]. Voisin [54] constructed a rational map $v: F(Y) \times F(Y) \rightarrow Z(Y)$ of degree 6, Chen [9] showed that the Voisin map $v$ can be resolved by blowing up the indeterminacy locus $Z=\left\{\left(L_{1}, L_{2}\right) \in F(Y) \times F(Y) \mid L_{1} \cap L_{2} \neq \emptyset\right\}$, and the blowup variety is a natural relative $Q u o t$-scheme over $Z(Y)$ if $Y$ is very general. The main theorem can be applied to this case, and implies that for any $k \geq 0$, there is an isomorphism of Chow groups

$$
\mathrm{CH}_{k}\left(\mathrm{Bl}_{Z}(F(Y) \times F(Y))\right) \simeq \mathrm{CH}_{k-1}(\widetilde{Z}) \oplus \mathrm{CH}_{k}(F(Y) \times F(Y)),
$$

where $\widetilde{Z}=\mathbb{P}\left(\omega_{Z}\right)$ is a Springer-type (partial) resolution of the indeterminacy locus $Z$, which is an isomorphism over $Z \backslash \Delta_{2}$, and a $\mathbb{P}^{1}$-bundle over the type II locus $\Delta_{2}=\left\{L \in \Delta \simeq F(Y) \mid \mathscr{N}_{L / Y} \simeq \mathscr{O}(1)^{\oplus 2} \oplus \mathscr{O}(-1)\right\}$ which is an algebraic surface (see Corollary 5.6).

The results of this paper could also be applied to many other situations of moduli spaces, for example, moduli of sheaves on surfaces [43, 44] and the moduli spaces of extensions of stable objects in K3 categories, which are generalizations of the varieties resolving Voisin's maps $[9,54]$. Another such example is provided by the pair of Thaddeus moduli spaces [48] $M_{C}(2, \mathscr{L}) \rightarrow N_{C}(2, \mathscr{L})$ and $M_{C}\left(2, \mathscr{L}^{\vee} \otimes \omega_{C}\right) \rightarrow N_{C}(2, \mathscr{L})$ studied by Koseki and Toda [29]. (Here, $\mathscr{L}$ is a line bundle of odd degree $d>0, N_{C}(2, \mathscr{L})$ is the moduli space of rank 2 semistable vector bundles over a curve $C$, with determinant $\mathscr{L}$, 
and $M_{C}(2, \mathscr{L})$ is the space $M_{\omega}$ of [48], where $\left.\omega=\left[\frac{d-1}{2}\right]\right)$. The results of this paper on flips (§3.2) and projectivizations (Theorem 4.1) would shed light on the study of the Chow theory of $N_{C}(2, \mathscr{L}){ }^{1}$

\subsection{Conventions}

Throughout this paper, $X$ is a Noetherian scheme of pure dimension, and $\mathscr{G}$ is a coherent sheaf over $X$. We say that $\mathscr{G}$ has rank $r$ if the rank of $\mathscr{G}(\eta):=\mathscr{G} \otimes \kappa(\eta)$ is $r$ at the generic point $\eta$ of each irreducible component of $X$. Assume that all schemes in consideration are defined over some fixed ground field $\mathbb{k}$. The terms 'locally free sheaves' and 'vector bundles' will be used interchangeably. We use Grothendieck's notations: for a coherent sheaf $\mathscr{F}$ on a scheme $X$, denote by $\mathbb{P}_{X}(\mathscr{F})=\operatorname{Proj}_{X} \operatorname{Sym}_{\mathscr{O}_{X}} \mathscr{F}$ its projectivization; we will write $\mathbb{P}(\mathscr{F})$ if the base scheme is clear from context. For a vector bundle $V$, we also use $\mathbb{P}_{\text {sub }}(V):=\mathbb{P}\left(V^{\vee}\right)$ to denote the moduli space of 1-dimensional linear subbundles of $V$.

For motives, we use the covariant convention of $[27,41,42,52,53]$. In particular, [27] contains a dictionary for translating between covariant and contravariant conventions. For a smooth projective variety $X$ over a field $\mathbb{k}$, denote by $\mathfrak{h}(X)$ its class $\left(X, \operatorname{Id}_{X}, 0\right)$ in Grothendieck's category of integral Chow motives of smooth projective varieties over $\mathbb{k}$. Notice that under the covariant convention, for a morphism $f: X \rightarrow Y$ of smooth projective varieties, $\Gamma_{f}$ induces the push-forward map $f_{*}: \mathfrak{h}(X) \rightarrow \mathfrak{h}(Y)$ and $\left[\Gamma_{f}^{t}\right]$ induces the pullback map $f^{*}: \mathfrak{h}(Y) \rightarrow \mathfrak{h}(X)(\operatorname{dim} Y-\operatorname{dim} X)$. Moreover, $\mathfrak{h}\left(\mathbb{P}^{1}\right)=1 \oplus \mathbb{L}=1 \oplus 1(1)$, where $1=\mathfrak{h}(\operatorname{Spec} k)$, and $\mathbb{L}=1(1)$ is the Lefschetz motive. In particular, the covariant Tate twist coincides with tensoring with $\mathbb{L}$ - that is, $\mathfrak{h}(X)(i)=\mathfrak{h}(X) \otimes \mathbb{L}^{i}$ for all $i \in \mathbb{Z}$. Furthermore, $\mathrm{CH}^{\ell}(\mathfrak{h}(X)(n))=\mathrm{CH}^{\ell-n}(X)$ and $\mathrm{CH}_{k}(\mathfrak{h}(X)(n))=\mathrm{CH}_{k-n}(X)$. We will use $h$ to denote the action $c_{1}(\mathscr{O}(1)) \cap\left(\_\right)$on motives when the line bundle $\mathscr{O}(1)$ is clear from the context.

\section{Preliminaries}

\subsection{Degeneracy loci}

Standard references are [18, 19, 20, 21, 34].

\section{Definition 2.1}

(1) Let $\mathscr{G}$ be a coherent sheaf of (generic) rank $r$ over a scheme $X$. For an integer $k \in \mathbb{Z}$, the degeneracy locus of $\mathscr{G}$ of rank $\geq k$ is defined to be

$$
X^{\geq k}(\mathscr{G}):=\{x \in X \mid \operatorname{rank} \mathscr{G}(x) \geq k\},
$$

where $\mathscr{G}(x):=\mathscr{G}_{x} \otimes_{\mathscr{O}_{X, x}} \kappa(x)$ is the fiber of $\mathscr{G}$ at $x \in X$. Notice that $X^{\geq k}(\mathscr{G})=X$ if $k \leq r$. We call $X_{\mathrm{sg}}(\mathscr{G}):=X^{\geq r+1}(\mathscr{G})$ the first degeneracy locus (or the singular locus) of $\mathscr{G}$.

\footnotetext{
${ }^{1}$ See [17] for recent results in this direction about rational Chow motives; our results here might also be helpful in obtaining results for integral coefficients.
} 
(2) Let $\sigma: \mathscr{F} \rightarrow \mathscr{E}$ be a morphism of $\mathscr{O}_{X}$-modules between locally free sheaves $\mathscr{F}$ and $\mathscr{E}$ on $X$. For an integer $\ell$, the degeneracy locus of $\sigma$ of rank $\ell$ is defined to be

$$
D_{\ell}(\sigma):=\{x \in X \mid \operatorname{rank} \sigma(x) \leq \ell\},
$$

where $\sigma(x):=\sigma_{x} \otimes_{\mathscr{O}_{X, x}} \kappa(x): \mathscr{F}(x) \rightarrow \mathscr{E}(x)$ is the map induced by $\sigma$ on the fibers.

The degeneracy loci $X^{\geq k}(\mathscr{G})$ and $D_{\ell}(\sigma)$ have natural closed subscheme structures given by Fitting ideals $[34, \S 7,2]$. The two notions are related as follows: let $\sigma: \mathscr{F} \rightarrow \mathscr{E}$ be an $\mathscr{O}_{X}$-module map between finite locally free sheaves and let $\mathscr{G}:=\operatorname{Coker}(\sigma)$ be the cokernel. Then $X^{\geq k}(\mathscr{G})=D_{\text {rank } \mathscr{E}-k}(\sigma)$ as closed subschemes of $X$.

The expected codimension of $D_{\ell}(\sigma) \subset X$ is $(\operatorname{rank} \mathscr{E}-\ell)(\operatorname{rank} \mathscr{F}-\ell)$ (if $\ell \leq$ $\min \{\operatorname{rank} \mathscr{E}$, rank $\mathscr{F}\})$. If $\mathscr{G}$ has homological dimension $\leq 1$ and rank $r$ - for example, if $\mathscr{G}=\operatorname{Coker}(\mathscr{F} \stackrel{\sigma}{\rightarrow} \mathscr{E})$ is the cokernel of an injective map of $\mathscr{O}_{X}$-modules between finite locally free sheaves - then for any $i \geq 0$, the expected codimension of $X^{\geq r+i}(\mathscr{G}) \subset X$ is $i(r+i)$.

In the universal local situation where $X=\operatorname{Hom}_{\mathbb{k}}(W, V)$ is the total space of maps between two vector spaces $W$ and $V$ over a field $\mathbb{k}$, there is a tautological map $\tau: W \otimes$ $\mathscr{O}_{X} \rightarrow V \otimes \mathscr{O}_{X}$ over $X$, such that $\tau(A)=A$ for $A \in \operatorname{Hom}(W, V)$.

Lemma 2.2. ([19, 20, 21]). Let $X=\operatorname{Hom}_{\mathbb{k}}(W, V)$ and denote $D_{\ell}=D_{\ell}(\tau) \subseteq X$ the degeneracy locus of the tautological map $\tau$ of rank $\ell$. Then for any $0 \leq \ell \leq \min \{\operatorname{rank} W, \operatorname{rank} V\}$, the singular locus of $D_{\ell}$ is $D_{\ell-1}$. Furthermore, for any regular point $A \in D_{\ell} \backslash D_{\ell-1}$, the following are true:

(1) The tangent space of $D_{\ell}$ at $A$ is $T_{A} D_{\ell}=\{T \in \operatorname{Hom}(W, V) \mid T(\operatorname{Ker} A) \subseteq \operatorname{Im} A\}$.

(2) The normal space of $D_{\ell}$ to $X$ at $A$ is $\left.N_{D_{\ell}} X\right|_{A}=\operatorname{Hom}(\operatorname{Ker} A, \operatorname{Coker} A)$.

Proof. See [19, §5.1, p. 54-55], [20, Lemma 4.12], or [21, Ex. V (4), p. 145].

In general, let $\sigma: \mathscr{F} \rightarrow \mathscr{E}$ be a map between vector bundles over a scheme $X$. For a fixed integer $\ell$, regarding the open degeneracy locus $D:=D_{\ell}(\sigma) \backslash D_{\ell-1}(\sigma)$ we have the following:

Lemma 2.3. Assume $X$ is a Cohen-Macaulay $\mathbb{k}$-scheme and $D:=D_{\ell}(\sigma) \backslash D_{\ell-1}(\sigma) \subset$ $X$ has the expected codimension $(\operatorname{rank} \mathscr{E}-\ell)(\operatorname{rank} \mathscr{F}-\ell)$. Then $\left.\sigma\right|_{D}:\left.\left.\mathscr{E}\right|_{D} \rightarrow \mathscr{F}\right|_{D}$ has constant rank $\ell$ over $D$, and $K:=\left.\operatorname{Ker} \sigma\right|_{D}$ and $C:=\left.\operatorname{Coker} \sigma\right|_{D}$ are locally free sheaves over $D$ of ranks rank $\mathscr{E}-\ell$ and rank $\mathscr{F}-\ell$, respectively. Moreover, $D \subset X$ is a locally complete intersection subscheme with normal bundle $N_{D / X} \simeq K^{\vee} \otimes C$.

Proof. First we prove the lemma for the total Hom space $H=\left|\operatorname{Hom}_{X}(\mathscr{F}, \mathscr{E})\right|$. Denote $\pi: H=\left|\operatorname{Hom}_{X}(\mathscr{F}, \mathscr{E})\right| \rightarrow X$ the projection, and let $\mathbb{D}_{\ell}:=D_{\ell}\left(\tau_{H}\right) \subset H$ be the degeneracy locus for the tautological map $\tau_{H}: \pi^{*} \mathscr{F} \rightarrow \pi^{*} \mathscr{E}$. As the statement is local, we may assume $X=\operatorname{Spec} A, \mathscr{F}=W \otimes_{\mathbb{k}} A$, and $\mathscr{E}=V \otimes_{\mathbb{k}} A$, where $A$ is a $\mathbb{k}$-algebra and $W, V$ are $\mathbb{k}$-vector spaces. Then $H=\operatorname{Hom}(W, V) \times_{\mathbb{k}} X$ is the flat base change of $\operatorname{Hom}_{\mathbb{k}}(W, V)$ along $X \rightarrow$ Spec $\mathbb{k}$, and $\mathbb{D}:=\mathbb{D}_{\ell} \backslash \mathbb{D}_{\ell-1}=D \times_{\mathbb{k}} X$. The desired result holds for $H$ and $\mathbb{D}$ by Lemma 2.2. 
In general, the map $\sigma: \mathscr{F} \rightarrow \mathscr{E}$ induces a section map $s_{\sigma}: X \rightarrow H$, such that $\sigma=s_{\sigma}^{*} \tau_{H}$ and $D=\mathbb{D} \times_{X} H$. Since $s$ is the section of a smooth separated morphism, it is a regular closed immersion. Since $H$ and $X$ are Cohen-Macaulay, $\mathbb{D} \hookrightarrow H$ is a regular immersion, and the intersection $D=\mathbb{D} \times_{X} H \hookrightarrow X$ has the expected codimension; therefore the inclusion $D \hookrightarrow X$ is also a regular immersion, with normal bundle $N_{D / X}=s_{\sigma}^{*} N_{\mathbb{D} / H}$. Finally, $s_{\sigma}^{*} N_{\mathbb{D} / H}=K^{\vee} \otimes C$ holds, since $K^{\vee}=s_{\sigma}^{*} \operatorname{Coker}\left(\tau_{H}^{\vee}\right)$ and $C=s_{\sigma}^{*} \operatorname{Coker}\left(\tau_{H}\right)$.

\subsection{Chow groups of projective bundles}

Let $X$ be a scheme and $\mathscr{E}$ a locally free sheaf of rank $r$ on $X$. Denote $\pi: \mathbb{P}(\mathscr{E}):=$ $\operatorname{Proj}\left(\operatorname{Sym}^{\bullet} \mathscr{E}\right) \rightarrow X$ the projection. Notice that our convention $\mathbb{P}(\mathscr{E})=\mathbb{P}_{\text {sub }}(\mathscr{E} \vee)$ is dual to Fulton's [18]. For simplicity, from now on we will denote $\zeta=c_{1}\left(\mathscr{O}_{\mathbb{P}(\mathscr{E})}(1)\right)$ and use the notation $\zeta^{i} \cdot \beta:=c_{1}\left(\mathscr{O}_{\mathbb{P}(\mathscr{E})}(1)\right)^{i} \cap \beta$, where $\beta \in \mathrm{CH}(\mathbb{P}(\mathscr{E}))$, to denote the cap product. For each $i \in[0, r-1]$, we introduce the following notations:

$$
\pi_{i}^{*}\left(\_\right)=\zeta^{i} \cdot \pi^{*}\left(\_\right): \mathrm{CH}_{k-(r-1)+i}(X) \rightarrow \mathrm{CH}_{k}(\mathbb{P}(\mathscr{E})), \quad \forall k \in \mathbb{Z} .
$$

The following results are summarized and deduced from [18, Proposition 3.1, Theorem 3.3] but presented in a way that fits better into our current work:

Theorem 2.4 (projective bundle formula).

(1) (Duality) For any $\alpha \in \mathrm{CH}(X)$,

$$
\pi_{*} \pi_{i}^{*}(\alpha)=\pi_{*}\left(c_{1}(\mathscr{O}(1))^{i} \cap \pi^{*}(\alpha)\right)= \begin{cases}0, & i<r-1 \\ \alpha, & i=r-1\end{cases}
$$

(2) For any $k \in \mathbb{N}$, there is an isomorphism of Chow groups:

$$
\bigoplus_{i=0}^{r-1} \pi_{i}^{*}: \bigoplus_{i=0}^{r-1} \mathrm{CH}_{k-(r-1)+i}(X) \stackrel{\sim}{\longrightarrow} \mathrm{CH}_{k}(\mathbb{P}(\mathscr{E})) .
$$

(3) The projection to the ith summand of this isomorphism is given by

$$
\pi_{i *}\left(\_\right)=\sum_{j=0}^{r-1-i}(-1)^{j} c_{j}(\mathscr{E}) \cap \pi_{*}\left(\zeta^{r-1-i-j} \cdot\left(\_\right), \quad \text { for } i=0,1, \ldots, r-1\right.
$$

Therefore for any $i, j \in[0, r-1]$, the following hold:

$$
\pi_{i *} \pi_{i}^{*}=\operatorname{Id}_{\mathrm{CH}(X)}, \quad \pi_{i *} \pi_{j}^{*}=0, i \neq j, \quad \operatorname{Id}_{\mathrm{CH}(\mathbb{P}(\mathscr{E}))}=\sum_{i=0}^{r-1} \pi_{i}^{*} \pi_{i *}
$$

Proof. Part (1) follows from [18, Proposition 3.1(a)], and part (2) follows from [18, Theorem 3.3], which could also be viewed as a special case of [18, Proposition 14.6.5]. For part (3), to agree with Fulton's notation, let $E=\mathscr{E} \vee$ be the dual vector bundle, so $\mathbb{P}_{\text {sub }}(E)=\mathbb{P}(\mathscr{E})$. From part $(2)$, for any $\beta \in \mathrm{CH}_{k}(\mathbb{P}(\mathscr{E}))$ there exist unique $\alpha_{i} \in$ 
$\mathrm{CH}_{k-(r-1)+i}(X), i \in[0, r-1]$, such that

$$
\beta=\sum_{i=0}^{r-1} \zeta^{i} \cdot \pi^{*} \alpha_{i}
$$

It follows from the definition of Segre classes $s_{i}(E) \cap \alpha:=\pi_{*}\left(\zeta^{i+r-1} \cdot \pi^{*} \alpha\right)$ that

$$
\pi_{*}\left(\zeta^{j} \cdot \beta\right)=\sum_{i=0}^{j} s_{i}(E) \cap \alpha_{r-1-j+i}, \quad \text { for } j=0,1, \ldots, r-1 .
$$

Then the desired results follow from solving $\alpha_{i}$ s using $1=c(E) s(E)=\left(1+c_{1}(E)+\right.$ $\left.c_{2}(E)+\cdots\right)\left(1+s_{1}(E)+s_{2}(E)+\cdots\right)$.

Notice that our maps $\pi_{i *}$ (resp., projectors $\pi_{i}^{*} \pi_{i *}$ ) are nothing but the explicit expressions of the correspondences $g_{i}$ (resp., orthogonal projectors $p_{r-i}$ ) that are inductively defined in [39, §7, p 457, Definition] (resp., [39, §7, p 456, Proposition]). By using these maps, Manin [39, §7, p 457] establishes an isomorphism of Chow motives:

$$
\bigoplus_{j=0}^{r-1} h^{r-1-j} \circ \pi^{*}: \bigoplus_{j=0}^{r-1} \mathfrak{h}(X)(j) \stackrel{\sim}{\longrightarrow} \mathfrak{h}(\mathbb{P}(\mathscr{E})) .
$$

Remark 2.5. The projector $\pi_{i *}$ can be expressed via the universal quotient bundle as

$$
\pi_{i *}=\pi_{*}\left(c_{r-1-i}\left(\mathcal{T}_{\mathbb{P}(\mathscr{E}) / X}(-1)\right) \cap\left(\_\right)\right): \mathrm{CH}(\mathbb{P}(\mathscr{E})) \rightarrow \mathrm{CH}(X) .
$$

This duality is explained for more general Grassmannian bundles in [23].

Remark 2.6 (change of basis). For any identification $\mathbb{P}(\mathscr{E}) \simeq \mathbb{P}(\mathscr{E} \otimes \mathscr{L})$, where $\mathscr{L} \in$ Pic $X$, denote $\zeta^{\prime}=c_{1}\left(\mathscr{O}_{\mathbb{P}(\mathscr{E} \otimes \mathscr{L})}(1)\right)=\zeta+\pi^{*} c_{1}(\mathscr{L})$, and $\pi_{i *}^{\prime}$ the projectors with respect to $\zeta^{\prime i} \cdot \pi^{*}\left(\_\right)$. Then the two bases $\left\{\zeta^{i}\right\}_{0 \leq i \leq r-1}$ and $\left\{\zeta^{\prime i}\right\}_{0 \leq i \leq r-1}$ differ by an invertible upper triangular change of basis. In particular, for any $0 \leq \bar{k} \leq r-1$, the following holds:

$$
\operatorname{Span}\left\{\zeta^{i} \mid 0 \leq i \leq k\right\}=\operatorname{Span}\left\{\zeta^{\prime i} \mid 0 \leq i \leq k\right\}
$$

where for any subset $\mathcal{S} \subset \mathrm{CH}^{*}(\mathbb{P}(\mathscr{E}))$, its span is defined by

$$
\operatorname{Span} \mathcal{S}:=\left\{\sum_{i} \alpha_{i} \cap \pi^{*} \beta_{i} \mid \alpha_{i} \in S, \beta_{i} \in \mathrm{CH}(X)\right\} .
$$

Similarly, for any $0 \leq k \leq r-1$, we can express $\pi_{k *}^{\prime}$ as a $\mathrm{CH}(X)$-linear combination of $\pi_{k *}, \pi_{k+1 *}, \ldots, \pi_{r-1 *}$, and vice versa.

Lemma 2.7 ([47, Lemma 5.3]). The following equality holds:

$$
c_{k}\left(\Omega_{\mathbb{P}(\mathscr{E}) / X}(1)\right)=\sum_{i=0}^{k}(-1)^{i} \zeta^{i} \cdot \pi^{*} c_{k-i}(\mathscr{E})=(-1)^{k} \sum_{i=0}^{k} \zeta^{i} \cdot \pi^{*} c_{k-i}\left(\mathscr{E}^{\vee}\right) .
$$




\subsection{Blowups}

Let $Z \subset X$ be a codimension $r \geq 2$ locally complete intersection subscheme. Denote $\pi$ : $\widetilde{X} \rightarrow X$ the blowup of $X$ along $Z$, with exceptional divisor $E \subset \widetilde{X}$. Then $E=\mathbb{P}\left(\mathscr{N}_{Z / X}^{\vee}\right)$ is a projective bundle over $Z$. We have a Cartesian diagram

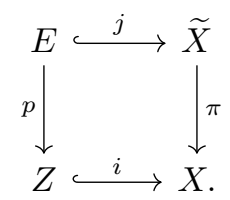

The excess bundle $\mathscr{V}$ for the diagram is defined by the short exact sequence

$$
0 \rightarrow \mathscr{N}_{E / \widetilde{X}} \rightarrow p^{*} \mathscr{N}_{Z / X} \rightarrow \mathscr{V} \rightarrow 0 .
$$

From the excess bundle formula [18, Theorem 6.3], one obtains the key formula for blowup:

$$
\pi^{*} i_{*}\left(\_\right)=j_{*}\left(c_{r-1}(\mathscr{V}) \cap p^{*}\left(\_\right)\right): \mathrm{CH}_{k}(Z) \rightarrow \mathrm{CH}_{k}(\widetilde{X}) .
$$

The following is summarized from [18, Proposition 6.7]:

Theorem 2.8 (blowups).

(1) The following hold:

$$
\pi_{*} \pi^{*}=\operatorname{Id}_{\mathrm{CH}(X)}, \quad p_{*}\left(c_{r-1}(\mathscr{V}) \cap p^{*}\left(\_\right)\right)=\operatorname{Id}_{\mathrm{CH}(Z)} .
$$

(2) For any $k \geq 0$, there exists a split short exact sequence

$$
0 \rightarrow \mathrm{CH}_{k}(Z) \stackrel{\left(c_{r-1}(\mathscr{V}) \cap p^{*}\left(\_\right),-i_{*}\right)}{\longrightarrow} \mathrm{CH}_{k}(E) \oplus \mathrm{CH}_{k}(X) \stackrel{(\varepsilon, \alpha) \mapsto j_{*} \varepsilon+\pi^{*} \alpha}{\longrightarrow} \mathrm{CH}_{k}(\widetilde{X}) \rightarrow 0,
$$

where a left inverse of the first map is given by $(\varepsilon, \alpha) \mapsto p_{*} \varepsilon$.

(3) This exact sequence induces an isomorphism of Chow groups

$$
\mathrm{CH}_{k}(X) \oplus \bigoplus_{i=0}^{r-2} \mathrm{CH}_{k-(r-1)+i}(Z) \stackrel{\sim}{\rightarrow} \mathrm{CH}_{k}(\tilde{X})
$$

given by $\left(\alpha, \oplus_{i=0}^{r-2} \beta_{i}\right) \mapsto \pi^{*} \alpha+j_{*}\left(\sum_{i=0}^{r-2} \zeta^{i} \cdot p^{*} \beta_{i}\right)$, where $\zeta=c_{1}\left(\mathscr{O}_{\mathbb{P}\left(\mathscr{N}_{Z / X}^{\vee}\right)}(1)\right)$.

Note that the well-known formula of part (3) follows from part (2) by the identification

$$
\begin{aligned}
\mathrm{CH}_{k}(\tilde{X}) & =\pi^{*} \mathrm{CH}_{k}(X) \oplus j_{*}\left(\mathrm{CH}_{k}(E)_{p_{*}=0}\right) \\
& =\pi^{*} \mathrm{CH}_{k}(X) \oplus \bigoplus_{i=0}^{r-2} j_{*}\left(\zeta^{i} \cdot p^{*} \mathrm{CH}_{k-(r-1)+i}(Z)\right),
\end{aligned}
$$

where $\mathrm{CH}_{k}(E)_{p_{*}=0}$ denotes the subgroup $\left\{\gamma \in \mathrm{CH}_{k}(E) \mid p_{*} \gamma=0\right\}$ of $\mathrm{CH}_{k}(E)$. A similar and more detailed argument is given later in the case of standard flips (see Theorem 3.6). There are similar results on Chow motives by Manin [39] (see also our Corollary 3.10). 


\section{The Cayley trick and standard flips}

The projectivization can be viewed as a combination of the situation of the Cayley trick and flips. In this section we study the Chow theory of the latter two cases.

\subsection{The Cayley trick and Chow groups}

The Cayley trick is a method to relate the geometry of the zero scheme of a regular section of a vector bundle to the geometry of a hypersurface (see the discussions of $[25, \S 2.3]$ ). The relationships for their derived categories were established by Orlov [46, Proposition 2.10]; we now focus on their Chow groups.

Let $\mathscr{E}$ be a locally free sheaf of rank $r \geq 2$ on a scheme $X$ and $s \in H^{0}(X, \mathscr{E})$ be a regular section, and denote $Z:=Z(s)$ the zero locus of the section $s$. Denote the projectivization by $q: \mathbb{P}(\mathscr{E})=$ ProjSym $\bullet \mathscr{E} \rightarrow X$. Then under the canonical identification

$$
H^{0}(X, \mathscr{E})=H^{0}\left(\mathbb{P}(\mathscr{E}), \mathscr{O}_{\mathbb{P}(\mathscr{E})}(1)\right)
$$

the section $s$ corresponds canonically to a section $f_{s}$ of $\mathscr{O}_{\mathbb{P}(\mathscr{E})}(1)$ on $\mathbb{P}(\mathscr{E})$. Denote the divisor defined by $f_{s}$ by

$$
\mathcal{H}_{s}:=Z\left(f_{s}\right) \subset \mathbb{P}(\mathscr{E})
$$

Then $\mathcal{H}_{s}=\mathbb{P}(\mathscr{G})=\operatorname{Proj} \operatorname{Sym}^{\bullet} \mathscr{G}$, where $\mathscr{G}=\operatorname{Coker}\left(\mathscr{O}_{X} \stackrel{s}{\longrightarrow} \mathscr{E}\right)$. Thus $\mathcal{H}_{s}$ is a $\mathbb{P}^{r-2}$-bundle over $X \backslash Z$, and a $\mathbb{P}^{r-1}$-bundle over $Z$. It follows that $\left.\mathcal{H}_{s}\right|_{Z}$ coincides with $\mathbb{P}_{Z}\left(\mathscr{N}_{i}\right)$, the projectivization of the normal bundle of inclusion $i: Z \hookrightarrow X$. The situation is illustrated in the following commutative diagram, with maps as labeled:

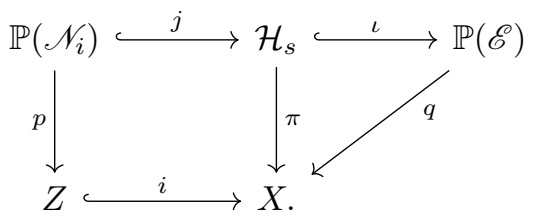

Since $\mathscr{N}_{i}=\mathscr{E}_{Z}$ and $\left.\mathscr{O}_{\mathbb{P}(\mathscr{E})}(1)\right|_{\mathbb{P}\left(\mathscr{N}_{i}\right)}=\mathscr{O}_{\mathbb{P}\left(\mathscr{N}_{i}\right)}(1)$, by abuse of notation we use $\zeta \cdot\left(\_\right)$to denote both $c_{1}\left(\mathscr{O}_{\mathbb{P}(\mathscr{E})}(1)\right) \cap\left(\_\right)$and $c_{1}\left(\mathscr{O}_{\mathbb{P}\left(\mathscr{N}_{i}\right)}(1)\right) \cap\left(\_\right)$. The main result of this section is the following:

Theorem 3.1 (the Cayley trick for Chow groups). There exists a split short exact sequence

$$
0 \rightarrow \bigoplus_{i=0}^{r-2} \mathrm{CH}_{k-(r-2)+i}(Z) \stackrel{f}{\rightarrow} \bigoplus_{i=0}^{r-2} \mathrm{CH}_{k-(r-2)+i}(X) \oplus \mathrm{CH}_{k}\left(\mathbb{P}\left(\mathscr{N}_{i}\right)\right) \stackrel{g}{\rightarrow} \mathrm{CH}_{k}\left(\mathcal{H}_{s}\right) \rightarrow 0
$$


where the maps $f$ and $g$ are given by

$$
\begin{aligned}
& f: \oplus_{i=0}^{r-2} \gamma_{i} \mapsto\left(-\oplus_{i=0}^{r-2} i_{*} \gamma_{i}, \sum_{i=0}^{r-2} \zeta^{i+1} \cdot p^{*} \gamma_{i}\right), \\
& g:\left(\oplus_{i=0}^{r-2} \alpha_{i}, \varepsilon\right) \mapsto \sum_{i=0}^{r-2} \zeta^{i} \cdot \pi^{*} \alpha_{i}+j_{*} \varepsilon
\end{aligned}
$$

where $p_{i *}$ is defined similarly to equation (2.1). A left inverse of $f$ is given by $\left(\oplus_{i=0}^{r-2} \alpha_{i}, \varepsilon\right) \mapsto$ $\oplus_{i=0}^{r-2} p_{i+1 *} \varepsilon$. Furthermore, the sequence induces an isomorphism

$$
\bigoplus_{i=0}^{r-2} \mathrm{CH}_{k-(r-2)+i}(X) \oplus \mathrm{CH}_{k-(r-1)}(Z) \stackrel{\sim}{\longrightarrow} \mathrm{CH}_{k}\left(\mathcal{H}_{s}\right),
$$

given by $\left(\oplus_{i=0}^{r-2} \alpha_{i}, \gamma\right) \mapsto \sum_{i=0}^{r-2} \zeta^{i} \cdot \pi^{*} \alpha_{i}+j_{*} p^{*} \gamma$, and in this decomposition the projection map to the first $(r-1)$-summands $\mathrm{CH}_{k}\left(\mathcal{H}_{s}\right) \rightarrow \mathrm{CH}_{k-(r-2)+i}(X), i=0,1, \ldots, r-2$, is given by $\beta \mapsto \pi_{i *} \beta$, where $\pi_{i *}$ is defined by equation (3.4) and the projection to the last summand $\mathrm{CH}_{k}\left(\mathcal{H}_{s}\right) \rightarrow \mathrm{CH}_{k-(r-1)}(Z)$ is given by $\beta \mapsto(-1)^{r-1} p_{*} j^{*} \beta$.

For simplicity, we introduce the following notation. For the projective bundles $q: \mathbb{P}(\mathscr{E}) \rightarrow$ $X$ and $p: \mathbb{P}\left(\mathscr{N}_{i}\right) \rightarrow Z$, similar to equation (2.1), we denote the projections to the $i$ th factors by

$$
q_{i *}: \mathrm{CH}_{k}(\mathbb{P}(\mathscr{E})) \rightarrow \mathrm{CH}_{k-(r-1)+i}(X), \quad p_{i *}: \mathrm{CH}_{k}\left(\mathbb{P}\left(\mathscr{N}_{i}\right)\right) \rightarrow \mathrm{CH}_{k-(r-1)+i}(Z),
$$

which are explicitly given as follows: for any $i=0,1, \ldots, r-1$,

$$
\begin{aligned}
& q_{i *}\left(\_\right)=\sum_{j=0}^{r-1-i}(-1)^{j} c_{j}(\mathscr{E}) \cap q_{*}\left(\zeta^{r-1-i-j} \cdot\left(\_\right)\right), \\
& p_{i *}\left(\_\right)=\sum_{j=0}^{r-1-i}(-1)^{j} c_{j}\left(\mathscr{N}_{i}\right) \cap p_{*}\left(\zeta^{r-1-i-j} \cdot\left(\_\right)\right) .
\end{aligned}
$$

Furthermore, for any $i \in[0, r-1], \alpha \in \mathrm{CH}(X)$, and $\gamma \in \mathrm{CH}(Z)$, we denote

$$
q_{i}^{*} \alpha:=\zeta^{i} \cdot q^{*} \alpha, \quad p_{i}^{*} \gamma:=\zeta^{i} \cdot p^{*} \gamma
$$

Then the projective bundle formula (Theorem 2.4) states the following:

(1) For all $i, j \in[0, r-1]$,

$$
q_{i *} q_{j}^{*}=\delta_{i, j} \operatorname{Id}_{\mathrm{CH}(X)}, \quad p_{i *} p_{j}^{*}=\delta_{i, j} \operatorname{Id}_{\mathrm{CH}(Z)} .
$$

(2) For all $\beta \in \mathrm{CH}(\mathbb{P}(\mathscr{E}))$ and $\varepsilon \in \mathrm{CH}\left(\mathbb{P}\left(\mathscr{N}_{i}\right)\right)$, the following relations hold:

$$
\beta=\sum_{i=0}^{r-1} q_{i}^{*} q_{i *} \beta \quad \varepsilon=\sum_{i=0}^{r-1} p_{i}^{*} p_{i *} \varepsilon .
$$

Now for all $\alpha \in \mathrm{CH}_{\ell}(X)$ and $\beta \in \mathrm{CH}_{k}\left(\mathcal{H}_{s}\right)$, and all $i \in[0, r-2]$, we define

$$
\pi_{i}^{*} \alpha:=\iota^{*} q_{i}^{*} \alpha \in \mathrm{CH}_{\ell+(r-2)-i}\left(\mathcal{H}_{s}\right), \quad \pi_{i *} \beta:=q_{i+1 *} \iota_{*} \beta \in \mathrm{CH}_{k-(r-2)+i}(X) .
$$


Then it follows from the projection formula that $\pi_{i}^{*} \alpha=\zeta^{i} \cdot \pi^{*} \alpha$, and $\pi_{r-2 *}=\pi_{*}$ and

$$
\pi_{i *}\left(\_\right)=\sum_{j=0}^{r-2-i}(-1)^{j} c_{j}(\mathscr{E}) \cap \pi_{*}\left(\zeta^{r-2-i-j} \cdot\left(\_\right)\right), \quad i=0, \ldots, r-2 .
$$

Notice that $c_{i}(\mathscr{E})=c_{i}(\mathscr{G})$ for $i \in[0, r-2]$, where $\mathscr{G}=\operatorname{Coker}\left(\mathscr{O}_{X} \stackrel{s}{\longrightarrow} \mathscr{E}\right)$; the relationships between $\pi_{i *} \mathrm{~s}$ and $\pi_{i}^{*} \mathrm{~s}$ are similar to the case of a $\mathbb{P}^{r-2}$-bundle.

We prove the theorem by the same steps as the blowup case in $[18, \S 6.7]$ :

Proposition 3.2 (compare [18, Proposition 6.7]).

(a) (Key formula). For all $\alpha \in \mathrm{CH}_{k}(Z)$,

$$
\pi^{*} i_{*} \alpha=j_{*}\left(\zeta \cdot p^{*} \alpha\right) \in \mathrm{CH}_{k+r-2}\left(\mathcal{H}_{s}\right) .
$$

Then by the projection formula, $\pi_{i}^{*} i_{*} \alpha=j_{*}\left(\zeta \cdot p_{i}^{*} \alpha\right)$ for all $i \in[0, r-2]$.

(b) For any $\alpha \in \mathrm{CH}_{k}(X), i, j \in[0, r-2]$, we have $\pi_{i *} \pi_{i}^{*} \alpha=\alpha, \pi_{i *} \pi_{j}^{*} \alpha=0$ if $i \neq j$.

(c) For $\varepsilon \in \mathrm{CH}\left(\mathbb{P}\left(\mathscr{N}_{i}\right)\right)$, if $j^{*} j_{*} \varepsilon=0$ and $p_{1 *} \varepsilon=\cdots=p_{r-1 *} \varepsilon=0$, then $\varepsilon=0$.

(d) (i) For any $\beta \in \mathrm{CH}_{k}\left(\mathcal{H}_{s}\right)$, there is an $\varepsilon \in \mathrm{CH}_{k}\left(\mathbb{P}\left(\mathscr{N}_{i}\right)\right)$ such that

$$
\beta=\sum_{i=0}^{r-2} \pi_{i}^{*} \pi_{i *} \beta+j_{*} \varepsilon
$$

(ii) For any $\beta \in \mathrm{CH}_{k}\left(\mathcal{H}_{s}\right)$, if $\pi_{i *} \beta=0, i \in[0, r-2]$, and $j^{*} \beta=0$, then $\beta=0$.

\section{Proof.}

(a) In fact, from [25, Remark. 2.5], the Euler sequence for $\mathbb{P}\left(\mathscr{N}_{i}\right)$ is equivalent to

$$
0 \rightarrow \mathscr{N}_{j} \rightarrow p^{*} \mathscr{N}_{i} \rightarrow \mathscr{O}_{\mathbb{P}\left(\mathscr{N}_{i}\right)}(1) \rightarrow 0
$$

where $\mathscr{N}_{i}=\left.\mathscr{E}\right|_{Z}$ and $\left.\mathscr{N}_{j} \simeq \Omega_{\mathbb{P}(\mathscr{E}) / X}(1)\right|_{\mathbb{P}\left(\mathscr{N}_{i}\right)}$. Therefore the excess bundle for diagram 3.1 is given by $\mathscr{O}_{\mathbb{P}\left(\mathscr{N}_{i}\right)}(1)$. Now from [18, Theorem 6.3, Propositions 6.2(1) and 6.6], one has:

$$
\pi^{*} i_{*}\left(\_\right)=j_{*} \pi_{\mathbb{P}\left(\mathscr{N}_{i}\right)}^{!}\left(\_\right)=j_{*}\left(c_{1}\left(\mathscr{O}_{\mathbb{P}\left(\mathscr{N}_{i}\right)}(1)\right) \cap p^{*}\left(\_\right)\right) .
$$

(b) Since $\iota: \mathcal{H}_{s} \hookrightarrow \mathbb{P}(\mathscr{E})$ is a divisor of $\mathscr{O}_{\mathbb{P}(\mathscr{E})}(1)$, we have $\iota^{*} \iota_{*}\left(\_\right)=\zeta \cdot\left(\_\right)$, and

$$
\pi_{i *} \pi_{j}^{*} \alpha=q_{i+1 *} \iota_{*}\left(\iota^{*}\left(q_{j}^{*} \alpha\right)\right)=q_{i+1 *}\left(\zeta \cdot \zeta^{j} \cdot q^{*} \alpha\right)=q_{i+1 *} q_{j+1}^{*} \alpha=\delta_{i, j} \alpha .
$$

(c) Since $j^{*} j_{*} \varepsilon=c_{r-1}\left(\mathscr{N}_{j}\right) \cap \varepsilon$, from the Euler sequence (3.5) and $\mathscr{N}_{i}=\left.\mathscr{E}\right|_{Z}$,

$$
c_{r-1}\left(\mathscr{N}_{j}\right)=\sum_{i=0}^{r-1}(-1)^{i} \zeta^{i} p^{*} c_{r-1-i}(\mathscr{E})=(-1)^{r-1} \zeta^{r-1}+\left(\text { lower order terms of } \zeta^{i}\right) .
$$

Therefore $j^{*} j_{*} \varepsilon=c_{r-1}\left(\mathscr{N}_{j}\right) \cap \varepsilon=0$ and $p_{1 *} \varepsilon=\cdots=p_{r-1 *} \varepsilon=0$ imply $p_{0 *} \varepsilon=p_{*}\left(\zeta^{r-1} \cdot \varepsilon\right)+$ $p_{*}\left(\left(\right.\right.$ lower-order terms of $\left.\left.\zeta^{i}\right) \cap \varepsilon\right)= \pm p_{*}\left(c_{r-1}\left(\mathscr{N}_{j}\right) \cap \varepsilon\right)+p_{*}\left(\left(\right.\right.$ lower-order terms of $\left.\zeta^{i}\right)$ $\cap \varepsilon)=0$. Hence $\varepsilon=\sum_{i=0}^{r-1} p_{i}^{*} p_{i *} \varepsilon=0$.

(d)(i) Over the open subscheme $U=X \backslash Z,\left.\mathcal{H}_{s}\right|_{\pi^{-1}(U)}=\mathbb{P}\left(\left.\mathscr{G}\right|_{U}\right)$ is a projective bundle with fiber $\mathbb{P}^{r-2}$. In fact, over $U$ there is an exact sequence of vector bundles $0 \rightarrow \mathscr{O}_{U} \rightarrow$ 
$\left.\left.\mathscr{E}\right|_{U} \rightarrow \mathscr{G}\right|_{U} \rightarrow 0$. Then the linear subbundle $\mathbb{P}\left(\left.\mathscr{G}\right|_{U}\right) \subset \mathbb{P}\left(\left.\mathscr{E}\right|_{U}\right)$ is a divisor representing the class $\zeta=c_{1}(\mathscr{O}(1))$. For any $\beta \in \mathrm{CH}\left(\mathbb{P}\left(\left.\mathscr{G}\right|_{U}\right)\right)$, by Theorem 2.4 applied to $\mathbb{P}\left(\mathscr{G}_{U}\right)$ there exists a unique $\alpha_{i} \in \mathrm{CH}(U)$ such that $\beta=\sum_{i=0}^{r-2}\left(\iota^{*} \zeta\right)^{i} \cdot \pi^{*} \alpha_{i}$. Therefore the following holds:

$$
\iota_{*} \beta=\sum_{i=0}^{r-2} \iota_{*}\left(\left(\iota^{*}(\zeta)^{i} \cdot \iota^{*}\left(q^{*} \alpha\right)\right)=\sum_{i=0}^{r-2} \zeta^{i+1} \cdot q^{*} \alpha_{i}\right.
$$

where the last equality follows from the projection formula and $\iota_{*} \iota^{*}\left(\_\right)=\zeta \cdot\left(\_\right)$. From the uniqueness statement of Theorem 2.4 applied to $\mathbb{P}(\mathscr{E})$, we know that

$$
\alpha_{i}=q_{i+1 *} \iota_{*} \beta=\pi_{i *} \beta .
$$

Therefore, over $U$, we have $\beta=\sum_{i=0}^{r-2} \pi_{i}^{*} \pi_{i *} \beta$. Now for any $\beta \in \mathrm{CH}_{k}\left(\mathcal{H}_{s}\right)$, $\left.\left(\beta-\sum_{i=0}^{r-2} \pi_{i}^{*} \pi_{i *} \beta\right)\right|_{U}=0$. From the exact sequence $\mathrm{CH}\left(\mathbb{P}\left(\mathscr{N}_{i}\right)\right) \rightarrow \mathrm{CH}\left(\mathcal{H}_{s}\right) \rightarrow$ $\mathrm{CH}\left(\left.\mathcal{H}_{s}\right|_{U}\right) \rightarrow 0$, there exists an $\varepsilon \in \mathrm{CH}\left(\mathbb{P}\left(\mathscr{N}_{i}\right)\right)$ such that $\beta-\sum_{i=0}^{r-2} \pi_{i}^{*} \pi_{i *} \beta=j_{*} \varepsilon$.

(d)(ii) From part (d)(i) we know that $\beta=j_{*} \varepsilon$, for $\varepsilon \in \mathrm{CH}_{k}\left(\mathbb{P}\left(\mathscr{N}_{i}\right)\right)$. Since the ambient square of diagram (3.1) is flat, by the flat base-change formula we have

$$
i_{*} p_{i+1 *}=q_{i+1 *}(\iota \circ j)_{*}=\pi_{i *} j_{*}, \quad \text { for } i=0,1, \ldots, r-2 .
$$

Therefore $i_{*}\left(p_{i+1 *} \varepsilon\right)=\pi_{i} * \beta=0$ for $i \in[0, r-2]$. Notice that since $\varepsilon=\sum_{i=0}^{r-1} p_{i}^{*} p_{i *} \varepsilon$, one has

$$
j_{*}\left(p_{0}^{*} p_{0 *} \varepsilon\right)=j_{*}(\varepsilon)-j_{*}\left(\zeta \cdot \sum_{i=0}^{r-2} p_{i}^{*} p_{i+1 *} \varepsilon\right)=j_{*}(\varepsilon)-\sum_{i=0}^{r-2} \pi_{i}^{*} i_{*}\left(p_{i+1 *} \varepsilon\right)=j_{*}(\varepsilon) .
$$

Here the second equality follows from the key formula of part (a). Now $j^{*} j_{*}\left(p_{0}^{*} p_{0 *} \varepsilon\right)=$ $j^{*} j_{*} \varepsilon=0$. By part (c), $p_{0}^{*} p_{0 *} \varepsilon=0$, hence $\beta=j_{*} \varepsilon=0$.

Theorem 3.1 follows from Proposition 3.2 as follows:

Proof of Theorem 3.1. The fact $g f=0$ follows from part (a). The surjectivity of $g$ is part (d)(i). By part (b), a left inverse of $f$ is given by $h:\left(\oplus_{i=0}^{r-2} \alpha_{i}, \varepsilon\right) \mapsto \oplus_{i=0}^{r-2} p_{i+1 *} \varepsilon$. In fact, $h f$ is

$$
\oplus_{i=0}^{r-2} \gamma_{i} \mapsto \oplus_{i=0}^{r-2} p_{i+1 *}\left(\sum_{j=0}^{r-2} \zeta^{j+1} \cdot p^{*} \gamma_{j}\right)=\oplus_{i=0}^{r-2}\left(p_{i+1 *} \sum_{j=0}^{r-2} p_{j+1}^{*} \gamma_{i}\right)=\oplus_{i=0}^{r-2} \gamma_{i} .
$$

To show the exactness of formula (3.2), suppose that for $\alpha_{i} \in \mathrm{CH}(X)$ and $\varepsilon \in \mathrm{CH}\left(\mathbb{P}\left(\mathscr{N}_{i}\right)\right)$, we have $\sum_{i=0}^{r-2} \pi_{i}^{*} \alpha_{i}+j_{*} \varepsilon=0$. Then similar to part (d)(ii), from part (b), for all $i \in[0, r-2]$,

$$
\alpha_{i}=-\pi_{i *}\left(j_{*} \varepsilon\right)=-i_{*} p_{i+1 *} \varepsilon \in \mathrm{CH}(X) .
$$

Now consider $\varepsilon^{\prime}=\varepsilon-\sum_{i=0}^{r-2} p_{i+1}^{*} p_{i+1 *} \varepsilon$. Then similar to the proof of part (d)(ii), we have

$$
j_{*} \varepsilon^{\prime}=j_{*}(\varepsilon)-j_{*}\left(\zeta \cdot \sum_{i=0}^{r-2} p_{i}^{*} p_{i+1 *} \varepsilon\right)=j_{*}(\varepsilon)-\sum_{i=0}^{r-2} \pi_{i}^{*} i_{*}\left(p_{i+1 *} \varepsilon\right)=j_{*}(\varepsilon)+\sum_{i=0}^{r-2} \pi_{i}^{*} \alpha_{i}=0,
$$


and $p_{1 *} \varepsilon^{\prime}=\cdots=p_{r-1 *} \varepsilon^{\prime}=0$ (since $\varepsilon^{\prime}=p_{0}^{*} p_{0 *} \varepsilon$ ). Therefore by part (c), $\varepsilon^{\prime}=0$. Hence $\left(\oplus_{i} \alpha_{i}, \varepsilon\right)=\left(-\oplus_{i} i_{*} \gamma_{i}, \sum_{i=0}^{r-2} p_{i+1}^{*} \gamma_{i}\right)$ for $\gamma_{i}=p_{i+1 *} \varepsilon$. Hence the sequence (3.2) is exact.

To prove the last statement, we show that for any $\beta \in \mathrm{CH}\left(\mathcal{H}_{s}\right)$, there exists a unique $\varepsilon \in \mathrm{CH}\left(\mathbb{P}\left(\mathscr{N}_{i}\right)\right)$ such that $p_{1 *} \varepsilon=\cdots=p_{r-1 *} \varepsilon=0$, and

$$
\beta=\sum_{i=0}^{r-2} \pi_{i}^{*} \pi_{i *} \beta+j_{*} \varepsilon .
$$

In fact, for any expression $\beta=\sum_{i=0}^{r-2} \pi_{i}^{*} \alpha_{i}+j_{*} \varepsilon$, by replacing $\varepsilon$ with $\varepsilon-\sum_{i=0}^{r-2} p_{i+1}^{*} p_{i+1 *} \varepsilon$ and $\alpha_{i}$ with $\alpha_{i}+i_{*}\left(p_{i+1 *} \varepsilon\right)$, we may assume $p_{1 *} \varepsilon=\cdots=p_{r-1 *} \varepsilon=0$. Hence by the projective bundle formula, $\varepsilon=p^{*} \gamma$ for a unique $\gamma \in \mathrm{CH}(Z)$. Now by the flat base-change formula,

$$
\pi_{i *}\left(j_{*} p^{*} \gamma\right)=q_{i+1 *}(\iota \circ j)_{*} p^{*} \gamma=q_{i+1 *} q^{*}\left(i_{*} \gamma\right)=0, \quad i \in[0, r-2]
$$

Therefore $\pi_{i *} \beta=\pi_{i *}\left(\sum_{i=0}^{r-2} \pi_{i}^{*} \alpha_{i}+j_{*} p^{*} \gamma\right)=\alpha_{i}$ for all $i=0,1, \ldots, r-2$. Hence we have established the identification

$$
\begin{aligned}
\mathrm{CH}_{k}\left(\mathcal{H}_{s}\right) & =\bigoplus_{i=0}^{r-2} \pi_{i}^{*} \mathrm{CH}_{k-(r-2)+i}(X) \oplus j_{*}\left(\mathrm{CH}_{k}\left(\mathbb{P}\left(\mathscr{N}_{i}\right)\right)_{p_{1 *}=\cdots=p_{r-1 *}=0}\right) \\
& =\bigoplus_{i=0}^{r-2} \pi_{i}^{*} \mathrm{CH}_{k-(r-2)+i}(X) \oplus j_{*} p^{*} \mathrm{CH}_{k-(r-1)}(Z)
\end{aligned}
$$

where $\mathrm{CH}_{k}\left(\mathbb{P}\left(\mathscr{N}_{i}\right)\right)_{p_{1 *}=\cdots=p_{r-1 *}=0}$ denotes the subgroup $\left\{\gamma \in \mathrm{CH}_{k}\left(\mathbb{P}\left(\mathscr{N}_{i}\right)\right) \mid p_{1 *} \gamma=\cdots=\right.$ $\left.p_{r-1 *} \gamma=0\right\}$ of $\mathrm{CH}_{k}\left(\mathbb{P}\left(\mathscr{N}_{i}\right)\right)$. Moreover, the projection maps to the first $(r-1)$-summands are respectively given by $\beta \mapsto \alpha_{i}=\pi_{i *} \beta$, for $i=0,1, \ldots, r-2$. For the formula of the projection to the last summand, it suffices to notice that $p_{*} j^{*} \pi_{i}^{*}\left(\_\right)=p_{*}\left(p_{i}^{*}\left(i^{*}\left(\_\right)\right)\right)=0$ for $i \in[0, r-2]$ and that $p_{*} j^{*} j_{*} p^{*}\left(\_\right)=p_{*}\left(c_{r-1}\left(\Omega_{\mathbb{P}(\mathscr{E})}(1)\right) \cap p^{*}\left(\_\right)\right)=(-1)^{r-1} \mathrm{Id}$.

Remark 3.3. If we denote $\Gamma=\mathbb{P}\left(\mathscr{N}_{i}\right)=\mathcal{H}_{s} \times_{X} Z$ and $\Gamma_{*}: \mathrm{CH}\left(\mathcal{H}_{s}\right) \rightarrow \mathrm{CH}(Z)$ (resp., $\Gamma^{*}: \mathrm{CH}(Z) \rightarrow \mathrm{CH}\left(\mathcal{H}_{s}\right)$ ) the map induced by the correspondence $[\Gamma] \in \mathrm{CH}\left(\mathcal{H}_{s} \times Z\right)$ (resp., by the transpose $[\Gamma]^{t} \in \mathrm{CH}\left(Z \times \mathcal{H}_{s}\right)$ of $\left.[\Gamma]\right)$, then

$$
\Gamma_{*}=p_{*} \circ j^{*} \text { and } \Gamma^{*}=j_{*} \circ p^{*} .
$$

In the foregoing proof, we have actually shown that the relations

$$
\Gamma_{*} \Gamma^{*}=(-1)^{r} \operatorname{Id}_{\mathrm{CH}(Z)}, \quad \pi_{i *} \pi_{j}^{*}=\delta_{i, j} \operatorname{Id}_{\mathrm{CH}(X)}, \quad \Gamma_{*} \pi_{i}^{*}=\pi_{i *} \Gamma^{*}=0
$$

hold for any $i, j \in[0, r-2]$, and that the isomorphism (3.3) is given by

$$
\operatorname{Id}_{\mathrm{CH}\left(\mathcal{H}_{s}\right)}=\sum_{i=0}^{r-2} \pi_{i}^{*} \pi_{i *}+\Gamma^{*} \Gamma_{*} .
$$


Corollary 3.4. If $X, \mathcal{H}_{s}$, and $Z$ are smooth and projective varieties over some ground field $\mathbb{k}$, then there is an isomorphism of Chow motives:

$$
\left(\bigoplus_{i=0}^{r-2} h^{r-2-i} \circ \pi^{*}\right) \oplus[\Gamma]^{t}:\left(\bigoplus_{i=0}^{r-2} \mathfrak{h}(X)(i)\right) \oplus \mathfrak{h}(Z)(r-1) \stackrel{\sim}{\longrightarrow} \mathfrak{h}\left(\mathcal{H}_{s}\right) .
$$

Proof. By Manin's identity principle, it suffices to notice that for any smooth $T$, the schemes $Z \times T \subset X \times T$ and $\mathcal{H}_{s} \times T$ are also in the same situation as the Cayley trick (Theorem 3.4). Hence the identities of Remark 3.3 hold for the Chow motives.

Example 3.5. Let $Y \subset \mathbb{P}^{n}$ be any complete intersection subvariety over a field $\mathbb{k}$ of codimension $c \geq 1$, say cut out by a regular section of the vector bundle $\bigoplus_{i=1}^{c} \mathscr{O}_{\mathbb{P}^{n}}\left(d_{i}\right)$. Following [28], if we fix a positive integer $r \geq \max \left\{\sum d_{i}-n-c, 1-c\right\}$, then $Y \subset \mathbb{P}^{n} \subset$ $\mathbb{P}^{n+r}=X$ is the zero subscheme of a regular section $s$ of the ample vector bundle

$$
\mathscr{E}:=\mathscr{O}_{\mathbb{P}^{n+r}}(1)^{\oplus r} \oplus \bigoplus_{i=1}^{c} \mathscr{O}_{\mathbb{P}^{n+r}}\left(d_{i}\right)
$$

It is shown in [28] that $F_{Y}:=\mathcal{H}_{s} \subset \mathbb{P}(\mathscr{E})$ is a Fano variety. Theorem 3.1 implies

$$
\mathrm{CH}_{*}\left(F_{Y}\right)=\mathrm{CH}_{*-r-c+1}(Y) \oplus \bigoplus_{i=0}^{r+c-2} \mathrm{CH}_{*-r-c+2+i}\left(\mathbb{P}^{n+r}\right)
$$

and similarly for Chow motives if we assume $Y$ is smooth. Hence the Chow group (resp., motive, rational Hodge structure if $\mathbb{k} \subset \mathbb{C}$ and $Y$ is smooth) of every complete intersection $Y$ can be split-embedded into that of a Fano variety $F_{Y}$, with complement given by copies of the Chow group (resp., motive, rational Hodge structure) of a projective space $\mathbb{P}^{n+r}$.

\subsection{Standard flips}

Let $(\Psi, \psi):(X, P) \rightarrow(\bar{X}, S)$ be a log-extremal contraction such that

(i) $P=\mathbb{P}_{S \text {, sub }}(F)$ for a vector bundle $F$ of rank $n+1$ on $S$ and

(ii) over every $s \in S,\left.\left(\mathscr{N}_{P / X}\right)\right|_{P_{s}} \simeq \mathscr{O}_{\mathbb{P}^{n}}(-1)^{\oplus(m+1)}$ for some fixed integer $m$.

By (the same argument as) [35, §1], there exists a vector bundle $F^{\prime}$ of rank $m+1$ such that $\mathscr{N}_{P / X}=\mathscr{O}_{\mathbb{P}_{\text {sub }}(F)}(-1) \otimes \psi^{*} F^{\prime}$. If we blow up $X$ along $P$, we get $\pi: \tilde{X} \rightarrow X$ with exceptional divisor $E=\mathbb{P}_{\text {sub }}\left(\mathscr{N}_{P / X}\right)=\mathbb{P}_{S \text {, sub }}(F) \times_{S} \mathbb{P}_{S \text {,sub }}\left(F^{\prime}\right)$. Furthermore, one can blow down $E$ along fibers of $\mathbb{P}_{S \text {, sub }}(F)$ and get $\pi^{\prime}: \widetilde{X} \rightarrow X^{\prime}$ and $\pi^{\prime}(E)=: P^{\prime} \simeq \mathbb{P}_{S \text {, sub }}\left(F^{\prime}\right)$, with $\mathscr{N}_{P^{\prime} / X^{\prime}} \simeq \mathscr{O}_{\mathbb{P}_{\text {sub }}\left(F^{\prime}\right)}(-1) \otimes \psi^{\prime *} F$, where $\psi^{\prime}: P^{\prime} \rightarrow S$ is the natural projection. Hence we obtain another log-extremal contraction $\left(\Psi^{\prime}, \psi^{\prime}\right):\left(X^{\prime}, P^{\prime}\right) \rightarrow(\bar{X}, S)$, which is birational to $(X, P)$.

The birational map $f: X \rightarrow X^{\prime}$ is called a standard (or ordinary) flip of type $(n, m)$. Note that $X>_{K} X^{\prime}$ (resp., $X \simeq_{K} X^{\prime}$ ) if and only if $n>m$ (resp., $n=m$ ). 
The geometry is illustrated in the following diagram, with maps as labeled:

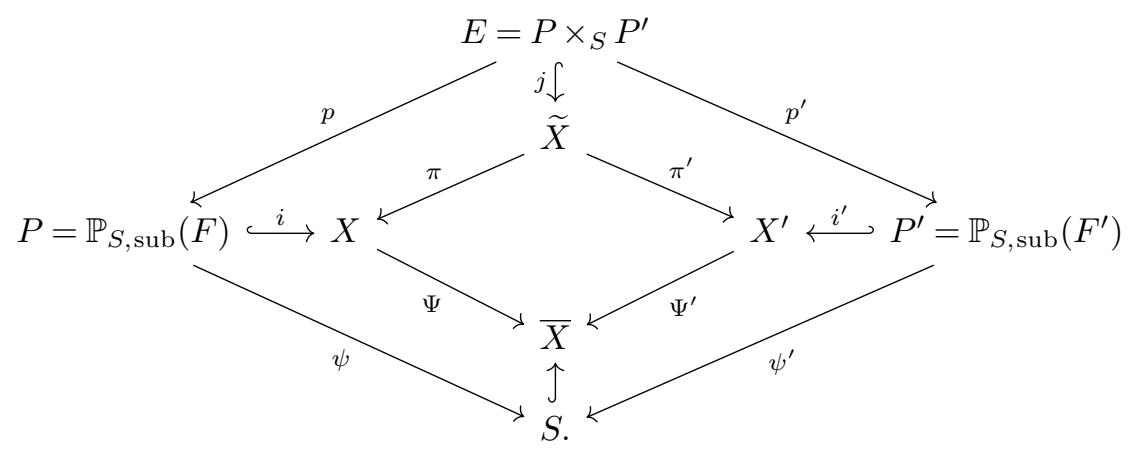

If $X>_{K} X^{\prime}$ (resp., $X \simeq_{K} X^{\prime}$ ), the expected relations of derived categories for the flip (resp., flop) $f: X \rightarrow X^{\prime}$ are established by Bondal and Orlov [8]. In this section we establish the corresponding relations on Chow groups, which complement the results of $[35, \S 3]$.

From now on we assume $n \geq m$ - that is, $X \geq_{K} X^{\prime}$. Denote $\Gamma$ the graph closure of $f$ in $X^{\prime} \times X$, which is nothing but $\widetilde{X}=X \times \bar{X} X^{\prime}$. Denote by $\Gamma_{*}: \mathrm{CH}_{k}(X) \rightarrow \mathrm{CH}_{k}\left(X^{\prime}\right)$ and $\Gamma^{*}: \mathrm{CH}_{k}\left(X^{\prime}\right) \rightarrow \mathrm{CH}_{k}(X)$ the maps induced by $[\Gamma] \in \mathrm{CH}_{\operatorname{dim} X}\left(X \times X^{\prime}\right)$. It is easy to see that

$$
\Gamma_{*}\left(\_\right)=\pi_{*}^{\prime} \pi^{*}\left(\_\right), \quad \Gamma^{*}\left(\_\right)=\pi_{*} \pi^{*}\left(\_\right) .
$$

Denote by $\mathscr{V}$ and $\mathscr{V}^{\prime}$ the respective excess bundles for the blowups $\pi: \widetilde{X} \rightarrow X$ and $\pi^{\prime}: \widetilde{X} \rightarrow$ $X^{\prime}$ - that is, they are defined by the short exact sequences

$$
0 \rightarrow \mathscr{N}_{E / \tilde{X}} \rightarrow p^{*} \mathscr{N}_{P / X} \rightarrow \mathscr{V} \rightarrow 0, \quad 0 \rightarrow \mathscr{N}_{E / \widetilde{X}} \rightarrow p^{\prime *} \mathscr{N}_{P^{\prime} / X^{\prime}} \rightarrow \mathscr{V}^{\prime} \rightarrow 0 .
$$

Denote by $\Phi_{*}: \mathrm{CH}_{k}(P) \rightarrow \mathrm{CH}_{k}\left(P^{\prime}\right)$ (resp., $\Phi^{*}: \mathrm{CH}_{k}\left(P^{\prime}\right) \rightarrow \mathrm{CH}_{k}(P)$ ) the maps given by the correspondence $c_{m}(\mathscr{V}) \in \mathrm{CH}^{m}\left(P \times{ }_{S} P^{\prime}\right)\left(\right.$ resp., $\left.c_{n}\left(\mathscr{V}^{\prime}\right) \in \mathrm{CH}^{n}\left(P^{\prime} \times{ }_{S} P\right)\right)$ - that is,

$$
\Phi_{*}\left(\_\right)=p_{*}^{\prime}\left(c_{m}(\mathscr{V}) \cap p^{*}\left(\_\right)\right) \quad \Phi^{*}\left(\_\right)=p_{*}\left(c_{n}\left(\mathscr{V}^{\prime}\right) \cap p^{*}\left(\_\right)\right) .
$$

It follows from the Euler sequence that $\mathscr{V}=\mathscr{O}_{P}(-1) \otimes \mathcal{T}_{P^{\prime} / S}(-1)$ and $\mathscr{V}^{\prime}=\mathcal{T}_{P / S}(-1) \otimes$ $\mathscr{O}_{P^{\prime}}(-1)$.

Theorem 3.6 (standard flips). Let $f: X \rightarrow X^{\prime}$ be a standard flip and assume $X^{\prime}$ is nonsingular and quasi-projective. Then we have the following:

(1) The following holds:

$$
\Gamma_{*} \Gamma^{*}=\operatorname{Id}_{\mathrm{CH}\left(X^{\prime}\right)}, \quad \Phi_{*} \Phi^{*}=\operatorname{Id}_{\mathrm{CH}\left(P^{\prime}\right)} .
$$

(2) There exists a split short exact sequence

$$
0 \rightarrow \mathrm{CH}_{k}\left(P^{\prime}\right) \stackrel{\left(\Phi^{*},-i_{*}^{\prime}\right)}{\longrightarrow} \mathrm{CH}_{k}(P) \oplus \mathrm{CH}_{k}\left(X^{\prime}\right) \stackrel{\left(\gamma, \alpha^{\prime}\right) \mapsto i_{*} \gamma+\Gamma^{*} \alpha^{\prime}}{\longrightarrow} \mathrm{CH}_{k}(X) \rightarrow 0,
$$

where a left inverse of the first map is given by $\left(\gamma, \alpha^{\prime}\right) \mapsto \Phi_{*} \gamma$. 
(3) This exact sequence induces an isomorphism of Chow groups

$$
\mathrm{CH}_{k}\left(X^{\prime}\right) \oplus \bigoplus_{i=0}^{n-m-1} \mathrm{CH}_{k-n+i}(S) \stackrel{\sim}{\longrightarrow} \mathrm{CH}_{k}(X),
$$

given by $\left(\alpha^{\prime}, \oplus_{i=0}^{n-m-1} \beta_{i}\right) \mapsto \Gamma^{*} \alpha^{\prime}+i_{*}\left(\sum_{i=0}^{n-m-1} \zeta^{i} \cdot \psi^{*} \beta_{i}\right)$. Furthermore, in this decomposition, the projection to the first summand is given by $\alpha \mapsto \alpha^{\prime}=\Gamma_{*} \alpha$.

Notice that in the flop case $m=n$, this result recovers the invariance of Chow groups under flops in [35]; and in the flip case $m<n$, this theorem completes the discussion of $[35, \S 2.3]$ by providing the complementary summands of the image of $\Gamma^{*}$ in the Chow group $\mathrm{CH}(X)$. Finally, as a blowup can be viewed as a standard flip of type $(n, 0)$, the theorem recovers the blowup formula in Theorem 2.8.

Proof of the first part of Theorem 3.6(1). The equality $\Gamma_{*} \Gamma^{*}=\mathrm{Id}$ follows exactly the same line of proof as [35, Theorem 2.1], as already mentioned in [35, §2.3]. We sketch the proof here for completeness. For any class $\left[W^{\prime}\right] \in \mathrm{CH}_{k}\left(X^{\prime}\right)$, by Chow's moving lemma (if allowing negative coefficients), we may assume it is represented by a cycle $W^{\prime}$ which intersects $P^{\prime}$ transversely. Therefore $\pi^{\prime *}\left[W^{\prime}\right]=[\widetilde{W}]$ by $[18$, Corollary 6.7.2], where $\widetilde{W}$ is the blowup of $W^{\prime}$ along $W^{\prime} \cap P^{\prime}$. Hence $\Gamma^{*}\left[W^{\prime}\right]=\pi_{*}[\widetilde{W}]=[W]$, where $W$ is the image of $\widetilde{W}$ and is also the proper transform of $W^{\prime}$ along the birational rational map $f^{-1}$. Now we have

$$
\pi^{*}[W]=[\widetilde{W}]+j_{*} \sum_{B}\left[E_{B}\right]
$$

where we let $B^{\prime} \subset W^{\prime} \cap P^{\prime}$ be a component; then $E_{B} \subset E$ are $k$-cycles supported over components $\bar{B}=\psi^{\prime}\left(B^{\prime}\right) \subset \psi^{\prime}\left(W^{\prime} \cap P^{\prime}\right) \subset S$. A direct computation of dimensions shows that for a general point $s$, the fiber $E_{B, s}$ over $s$ has dimension

$$
\operatorname{dim} E_{B, s} \geq \operatorname{dim} E_{B}-\operatorname{dim}(\bar{B}) \geq \operatorname{dim} E_{B}-\operatorname{dim}\left(B^{\prime}\right)=k-(k-(n+1))=n+1 .
$$

Now $E_{B, s}$ must contain positive fibers of of $p_{s}^{\prime}: \mathbb{P}_{s}^{n} \times \mathbb{P}_{s}^{m} \rightarrow \mathbb{P}_{s}^{m}$, as $n+1>n \geq m$. Hence $\pi_{*} j_{*}\left[E_{B}\right]=p_{*}\left[E_{B}\right]=0$, and $\Gamma_{*} \Gamma^{*}\left[W^{\prime}\right]=\pi_{*}^{\prime} \pi^{*}[W]=\pi_{*}^{\prime}[\widetilde{W}]=\left[W^{\prime}\right]$.

Remark 3.7. Notice that this argument does not work in the other direction for $\Gamma^{*} \Gamma_{*}[V]$, where $[V] \in \mathrm{CH}_{k}(X)$. The reason is as follows: the fiber $E_{B^{\prime}, s}$ of the $k$-cycle $E_{B^{\prime}}$ in $\pi^{\prime *} \Gamma^{*}[V]=\pi^{\prime *}\left[V^{\prime}\right]=\widetilde{V}+j_{*} \sum E_{B^{\prime}}$ has dimension $\geq m+1$, but $m \leq n$, and thus $E_{B^{\prime}, s}$ is not necessarily contracted by $p_{*}$. However, if $k \leq m$ (in which case we may assume $V \cap P=\emptyset)$ or $k \geq n+1+\operatorname{dim} \psi(V \cap S)-$ for example, if $k \geq \operatorname{dim} S+n+1-$ then the argument still works:

$$
\Gamma^{*} \Gamma_{*}[V]=[V] \quad \text { if } k \leq m+1 \text { or } k \geq n+\operatorname{dim} \psi(V \cap S) .
$$

For the intermediate cases $m+1 \leq k \leq n+\operatorname{dim} \psi(V \cap S) \leq n+\operatorname{dim} S$, the same argument implies only $\Gamma^{*} \Gamma_{*}[V]=[V]+i_{*} \sum_{Z \subset P}[Z]$ for certain cycles $Z \subset P$ supported on $P$; these cycles will be precisely explained by statements (2) and (3) of the theorem. 
Proof of the second part of Theorem 3.6(1). It follows from Lemma 2.7 that

$$
\begin{aligned}
c_{m}(\mathscr{V}) & =(-1)^{m} c_{m}\left(\mathscr{O}_{P}(1) \otimes \Omega_{P^{\prime} / S}(1)\right)=(-1)^{m} \sum_{t=0}^{m} \zeta^{t} \cdot c_{m-t}\left(\Omega_{P^{\prime} / S}(1)\right) \\
& =(-1)^{m} \sum_{t=0}^{m}(-1)^{m-t} \sum_{s=0}^{m-t} c_{s}\left(F^{\prime}\right) \cdot\left(\zeta^{\prime}\right)^{m-s-t} \cdot \zeta^{t}
\end{aligned}
$$

and

$$
\begin{aligned}
c_{n}\left(\mathscr{V}^{\prime}\right) & =(-1)^{n} c_{n}\left(\Omega_{P / S}(1) \otimes \mathscr{O}_{P^{\prime}}(1)\right)=(-1)^{n} \sum_{j=0}^{n} c_{n-j}\left(\Omega_{P / S}(1)\right) \cdot\left(\zeta^{\prime}\right)^{j} \\
& =(-1)^{n} \sum_{j=0}^{n}(-1)^{n-j} \sum_{i=0}^{n-j} c_{i}(F) \cdot \zeta^{n-i-j} \cdot\left(\zeta^{\prime}\right)^{j} .
\end{aligned}
$$

The map $\Phi_{*} \circ \Phi^{*}$ is given by the convolution of correspondences

$$
c_{m}(\mathscr{V}) * c_{n}\left(\mathscr{V}^{\prime}\right):=p_{13 *}\left(p_{12}^{*}\left(c_{n}\left(\mathscr{V}^{\prime}\right)\right) \cdot p_{23}^{*}\left(c_{m}(\mathscr{V})\right)\right) \in \mathrm{CH}^{m}\left(P^{\prime} \times_{S} P^{\prime}\right),
$$

where $p_{i j}$ are the obvious projections from $P^{\prime} \times{ }_{S} P \times{ }_{S} P^{\prime}$ to the corresponding factors and the cohomological degree $m$ is computed via $m+n-\operatorname{dim}(P / S)=m$. To avoid confusion, we denote the product $P^{\prime} \times{ }_{S} P \times{ }_{S} P^{\prime}$ by $P_{1}^{\prime} \times{ }_{S} P \times_{S} P_{2}^{\prime}$, and denote the relative $\mathscr{O}(1)$ classes of $P_{1}^{\prime}$ and $P_{2}^{\prime}$ by $\zeta_{1}^{\prime}$ and $\zeta_{2}^{\prime}$, respectively. Therefore,

$$
c_{m}(\mathscr{V}) * c_{n}\left(\mathscr{V}^{\prime}\right)=p_{13} *\left(\sum_{j=0}^{n} \sum_{t=0}^{m}(-1)^{j+t} \sum_{s=0}^{m-t} \sum_{i=0}^{n-j} c_{s}\left(F_{2}^{\prime}\right) \cdot c_{i}(F) \cdot \zeta^{n+t-i-j} \cdot\left(\zeta_{1}^{\prime}\right)^{j} \cdot\left(\zeta_{2}^{\prime}\right)^{m-s-t}\right) .
$$

Since $p_{13 *}\left(\zeta^{k}\right)=0$ for all $0 \leq k \leq n-1$, the only terms inside the parentheses that could survive $p_{13 *}$ are the ones whose indices satisfy $t-i-j \geq 0$. Thus we may assume that the indices of the summation satisfy $j \leq t \leq m$ and $0 \leq i \leq t-j$. From the definition of the Segre class of $F$, we have $p_{13 *}\left(\zeta^{n+k}\right)=s_{k}(F)$, hence

$$
c_{m}(\mathscr{V}) * c_{n}\left(\mathscr{V}^{\prime}\right)=\sum_{j=0}^{m} \sum_{t=j}^{m}(-1)^{j+t} \sum_{s=0}^{m-t} \sum_{i=0}^{t-j} c_{i}(F) \cdot s_{t-i-j}(F) \cdot c_{s}\left(F_{2}^{\prime}\right) \cdot\left(\zeta_{1}^{\prime}\right)^{j} \cdot\left(\zeta_{2}^{\prime}\right)^{m-s-t} .
$$

From $c(F) s(F)=1$, we know that $\sum_{i=0}^{t-j} c_{i}(F) \cdot s_{t-i-j}(F)=0$ unless $t=j$, in which case $c_{0}(F) s_{0}(F)=1$. Hence this expression reduces to

$$
\begin{aligned}
c_{m}(\mathscr{V}) * c_{n}\left(\mathscr{V}^{\prime}\right) & =\sum_{j=0}^{m} \sum_{s=0}^{m-j} c_{s}\left(F_{2}^{\prime}\right) \cdot\left(\zeta_{1}^{\prime}\right)^{j} \cdot\left(\zeta_{2}^{\prime}\right)^{m-j-s}=\sum_{j=0}^{m} c_{m-j}\left(\mathcal{T}_{P_{2}^{\prime} / S}(-1)\right) \cdot\left(\zeta_{1}^{\prime}\right)^{j} \\
& =c_{m}\left(\mathscr{O}_{P_{1}^{\prime}}(1) \otimes \mathcal{T}_{P_{2}^{\prime} / S}(-1)\right) .
\end{aligned}
$$

(For the second equality, we used Lemma 2.7.) On the other hand, the diagonal $\Delta_{P^{\prime}} \subset$ $P^{\prime} \times{ }_{S} P^{\prime}$ is the zero locus of a regular section $s$ of the rank $m$ vector bundle $\mathscr{O}_{P^{\prime}}(1) \otimes$ $\mathcal{T}_{P^{\prime} / S}(-1)$; the section $s$ under the canonical identification

$$
\Gamma\left(P^{\prime} \times_{S} P^{\prime}, \mathscr{O}_{P^{\prime}}(1) \otimes \mathcal{T}_{P^{\prime} / S}(-1)\right)=\Gamma\left(S, F^{\prime \vee} \otimes F^{\prime}\right)=\operatorname{Hom}_{S}\left(F^{\prime}, F^{\prime}\right)
$$


corresponds to $1_{F^{\prime}}: F^{\prime} \rightarrow F^{\prime}$. Hence $\left[\Delta_{P^{\prime}}\right]=c_{m}\left(\mathscr{O}_{P^{\prime}}(1) \otimes \mathcal{T}_{P^{\prime} / S}(-1)\right)$, and therefore

$$
c_{m}(\mathscr{V}) * c_{n}\left(\mathscr{V}^{\prime}\right)=\left[\Delta_{P^{\prime}}\right], \quad \text { hence } \Phi_{*} \Phi^{*}=\operatorname{Id}_{\mathrm{CH}\left(P^{\prime}\right)} .
$$

Before proceeding the rest of the proof of Theorem 3.6, we study more about the maps $\Phi_{*}$ and $\Phi^{*}$. First, notice that the projective bundle formula (Theorem 2.4) can be regarded as equipping $\mathrm{CH}(P)$ and $\mathrm{CH}\left(P^{\prime}\right)$ with natural 'free module structures over $\mathrm{CH}(S)$ ':

\section{Lemma 3.8.}

(1) The maps $\Phi_{*}: \mathrm{CH}(P) \rightarrow \mathrm{CH}\left(P^{\prime}\right)$ and $\Phi^{*}: \mathrm{CH}\left(P^{\prime}\right) \rightarrow \mathrm{CH}(P)$ are ${ }^{\circ} \mathrm{CH}(S)$-linear' that is, for all $\alpha \in \mathrm{CH}^{*}(P), \alpha^{\prime} \in \mathrm{CH}^{*}\left(P^{\prime}\right)$, and $\theta \in \mathrm{CH}_{*}(S)$,

$$
\Phi_{*}\left(\alpha \cap \psi^{*} \theta\right)=\Phi_{*}(\alpha) \cap \psi^{\prime *} \theta, \quad \Phi^{*}\left(\alpha^{\prime} \cap \psi^{\prime *} \theta\right)=\Phi^{*}\left(\alpha^{\prime}\right) \cap \psi^{*} \theta .
$$

(2) Consider the following 'sub- $\mathrm{CH}(S)$-modules' of $\mathrm{CH}(P)$ :

$$
\begin{aligned}
\mathrm{CH}(P)_{m} & :=\operatorname{Span}\left\{\zeta^{n-m}, \zeta^{n-m+1}, \ldots, \zeta^{m}\right\} \\
& =\zeta^{n-m} \cdot \mathrm{CH}(S) \oplus \cdots \oplus \zeta^{m} \cdot \mathrm{CH}(S) \subset \mathrm{CH}(P), \\
\mathrm{CH}(P)_{\Phi_{*}}=0 & :=\operatorname{Span}\left\{1, \zeta, \ldots, \zeta^{n-m-1}\right\}=1 \cdot \mathrm{CH}(S) \oplus \cdots \oplus \zeta^{n-m-1} \cdot \mathrm{CH}(S) \subset \mathrm{CH}(P) .
\end{aligned}
$$

Then $\Phi_{*}$ is injective on $\mathrm{CH}(P)_{m}$, and with image $\Phi_{*}\left(\mathrm{CH}(P)_{m}\right)=\mathrm{CH}\left(P^{\prime}\right)$. Furthermore, $\Phi_{*}$ is zero on $\mathrm{CH}(P)_{\Phi_{*}=0}$.

(3) $\Phi^{*}$ is injective, and its image $\operatorname{Im}\left(\Phi^{*}\right)$ satisfies $\mathrm{CH}(P)_{\Phi_{*}=0} \simeq \mathrm{CH}(P) / \operatorname{Im}\left(\Phi^{*}\right)$.

Proof. Statement (1) follows directly from the projection formula [18, Theorem 3.2(c)] and Theorem 2.4(1). For statement (2), notice that for any $0 \leq i \leq m$,

$$
\begin{aligned}
\Phi_{*}\left(\zeta^{n-m+i}\right) & =p_{*}^{\prime}\left(c_{m}(\mathscr{V}) \cup p^{*} \zeta^{n-m+i}\right) \\
& =p_{*}^{\prime}\left((-1)^{m} \sum_{t=0}^{m}(-1)^{m-t} \sum_{s=0}^{m-t} c_{s}\left(F^{\prime}\right) \cdot\left(\zeta^{\prime}\right)^{m-s-t} \cdot \zeta^{n-m+i+t}\right) \\
& =\sum_{t=m-i}^{m}(-1)^{t} \sum_{s=0}^{m-t} c_{s}\left(F^{\prime}\right) \cdot s_{t-(m-i)}(F) \cdot\left(\zeta^{\prime}\right)^{m-s-t} \\
& =(-1)^{m-i}\left(\zeta^{\prime}\right)^{i}+(\text { lower-order terms }) .
\end{aligned}
$$

(For example, $\Phi_{*}\left(\zeta^{m}\right)=\left(\zeta^{\prime}\right)^{n}+$ (lower-order terms) and $\Phi_{*}\left(\zeta^{n}\right)=(-1)^{n}\left(\zeta^{\prime}\right)^{0}$.) This computation together with statement (1) shows that $\Phi_{*}\left(\zeta^{n-m+i} \cap \psi^{*} \theta\right)=$ $\left( \pm \zeta^{\prime i}+(\right.$ lower-order terms $\left.)\right) \cap \psi^{\prime *} \theta$ for all $\theta \in \mathrm{CH}(S)$, which implies the injectivity of $\Phi_{*}$ on $\mathrm{CH}(P)_{m}$. The same computation in the case $i<0$ shows that $\Phi_{*}$ is zero on $\mathrm{CH}(P)_{\Phi_{*}=0}$. A similar computation shows

$$
\Phi^{*}\left(\zeta^{\prime i}\right)= \pm \zeta^{n-m+i}+(\text { lower-order terms })
$$

for $0 \leq i \leq m$, which implies statement (3).

Lemma 3.9. For any $\gamma \in \mathrm{CH}(P)$, if $\Phi_{*}(\gamma)=0$ and $i^{*} i_{*} \gamma=0$, then $\gamma=0$. 
Proof. Let $\gamma=\sum_{i=0}^{n} \zeta^{i} \cdot \psi^{*} \theta_{i}$ for $\theta_{i} \in \mathrm{CH}(S)$. Then from the lemma, $\Phi_{*} \gamma=0$ implies $\theta_{n-m}=\theta_{n-m+1}=\cdots=\theta_{n}=0$. On the other hand, $i^{*} i_{*} \gamma=c_{m+1}\left(\mathscr{N}_{P / X}\right) \cap \gamma=c_{m+1}\left(F^{\prime} \otimes\right.$ $\left.\mathscr{O}_{P}(-1)\right) \cap \gamma=0$, and

$$
\begin{aligned}
c_{m+1}\left(F^{\prime} \otimes \mathscr{O}_{P}(-1)\right) & =\sum_{i=0}^{m+1} c_{m+1-i}\left(F^{\prime}\right)(-\zeta)^{i} \\
& =(-1)^{m+1} \zeta^{m+1}+\text { (lower-order terms) } .
\end{aligned}
$$

Hence by the uniqueness of an expression of the form $\sum_{i=0}^{n} \zeta^{i} \cdot \psi^{*}\left(\_\right)$, one can inductively show $\theta_{n-m-1}=0, \theta_{n-m-2}=0, \ldots, \theta_{0}=0$. Therefore $\gamma=0$.

Proof of Theorem 3.6(2). To show that the sequence is a complex, simply observe that for any $\gamma^{\prime} \in \mathrm{CH}(P)$,

$$
\begin{aligned}
i_{*} \Phi^{*} \gamma^{\prime}+\Gamma^{*}\left(-i_{*}^{\prime} \gamma^{\prime}\right) & =i_{*} p_{*}\left(c_{n}\left(\mathscr{V}^{\prime}\right) \cap p^{\prime *} \gamma^{\prime}\right)-\Gamma^{*} i_{*}^{\prime} \gamma^{\prime} \\
& =\pi_{*} j_{*}\left(c_{n}\left(\mathscr{V}^{\prime}\right) \cap p^{\prime *} \gamma^{\prime}\right)-\Gamma^{*} i_{*}^{\prime} \gamma^{\prime} \\
& \stackrel{\text { (k.f.) }}{=} \pi_{*} \pi^{\prime *} i_{*}^{\prime} \gamma^{\prime}-\pi_{*} \pi^{* *} i_{*}^{\prime} \gamma^{\prime}=0 .
\end{aligned}
$$

(Here and later, '(k.f.)' means the key formula (2.2) for blowup.)

For any $\alpha \in \mathrm{CH}_{k}(X), \alpha-\Gamma^{*} \Gamma_{*} \alpha=0$ on $\mathrm{CH}_{k}(X \backslash P)$. Then from the exact sequence $\mathrm{CH}_{k}(P) \rightarrow \mathrm{CH}_{k}(X) \rightarrow \mathrm{CH}_{k}(X \backslash P) \rightarrow 0$, there exists an element $\gamma \in \mathrm{CH}_{k}(P)$ such that $\alpha=\Gamma^{*} \Gamma_{*} \alpha+i_{*} \gamma$. This establishes the surjectivity of the last map of the sequence of Theorem 3.6(2). The injectivity of the first map and the left inverse statement follow directly from $\Phi_{*} \Phi_{*}=$ Id. To show that the sequence is exactness in the middle, assume $\left(\gamma, \alpha^{\prime}\right) \in \mathrm{CH}(P) \oplus \mathrm{CH}\left(X^{\prime}\right)$ such that $i_{*} \gamma+\Gamma^{*} \alpha^{\prime}=0$; we want to find $\gamma^{\prime}$ such that $\left(\gamma, \alpha^{\prime}\right)=$ $\left(\Phi^{*} \gamma^{\prime},-i_{*}^{\prime} \gamma\right)$, since

$$
\begin{aligned}
\alpha^{\prime} & =\Gamma_{*} \Gamma^{*} \alpha^{\prime}=-\Gamma_{*} i_{*} \gamma=-\pi_{*}^{\prime} \pi^{*} i_{*} \gamma \stackrel{\text { (k.f.) }}{=}-\pi_{*}^{\prime} j_{*}\left(c_{m}(\mathscr{V}) \cap p^{*} \gamma\right) \\
& =-i_{*}^{\prime} p_{*}^{\prime}\left(c_{m}(\mathscr{V}) \cap p^{*} \gamma\right)=-i_{*}^{\prime} \Phi_{*}(\gamma)
\end{aligned}
$$

Define $\gamma_{0}=\gamma-\Phi^{*} \Phi_{*} \gamma$. The goal is to show $\gamma_{0}=0$. Notice that

$$
\Phi_{*} \gamma_{0}=\Phi_{*} \gamma-\Phi_{*} \Phi^{*} \Phi_{*} \gamma=0
$$

and

$$
\begin{aligned}
i_{*} \gamma_{0} & =i_{*} \gamma-i_{*} \Phi^{*} \Phi_{*} \gamma=i_{*} \gamma-i_{*} p_{*}\left(c_{n} \mathscr{V}^{\prime} \cap p^{*}\left(\Phi^{*} \gamma\right)\right) \\
& =i_{*} \gamma-\pi_{*} j_{*}\left(c_{n} \mathscr{V}^{\prime} \cap p^{\prime *}\left(\Phi_{*} \gamma\right)\right) \stackrel{(\mathrm{k.f.})}{=} i_{*} \gamma-\pi_{*} \pi^{\prime *} i_{*}^{\prime} \Phi_{*} \gamma \\
& =i_{*} \gamma-\Gamma^{*}\left(i_{*}^{\prime} \Phi_{*} \gamma\right)=i_{*} \gamma+\Gamma^{*} \alpha^{\prime}=0
\end{aligned}
$$

From Lemma 3.9, $\gamma_{0}=0$, hence $\left(\gamma, \alpha^{\prime}\right)=\left(\Phi^{*} \gamma^{\prime},-i_{*}^{\prime} \gamma\right)$ for $\gamma^{\prime}=\Phi_{*} \gamma$. 
Proof of Theorem 3.6(3). As before, from Lemma 3.8(3) and the exact sequence of part (2), we obtain that for any $\alpha \in \mathrm{CH}_{k}(X)$, there exist an $\alpha^{\prime} \in \mathrm{CH}_{k}(X)$ and a unique $\gamma \in \mathrm{CH}_{k}(P)$ such that $\Phi_{*} \gamma=0$ and $\alpha=\Gamma^{*} \alpha^{\prime}+i_{*} \gamma$. Further notice that

$$
\Gamma_{*} i_{*} \gamma=\pi_{*}^{\prime} \pi^{*} i_{*} \gamma \stackrel{\text { (k.f.) }}{=} \pi_{*}^{\prime} j_{*}\left(c_{n}(\mathscr{V}) \cap p^{*} \gamma\right)=i_{*}^{\prime} p_{*}^{\prime} j_{*}\left(c_{n}(\mathscr{V}) \cap p^{*} \gamma\right)=i_{*}^{\prime} \Phi_{*} \gamma=0
$$

Therefore $\alpha^{\prime}=\Gamma_{*} \alpha$. Hence we have established

$$
\begin{aligned}
\mathrm{CH}_{k}(X) & =\Gamma^{*} \mathrm{CH}_{k}\left(X^{\prime}\right) \oplus i_{*}\left(\mathrm{CH}(P)_{\Phi_{*}=0}\right) \\
& =\Gamma^{*} \mathrm{CH}_{k}\left(X^{\prime}\right) \oplus i_{*}\left(\bigoplus_{i=0}^{n-m-1} \zeta^{i} \cdot \psi^{*} \mathrm{CH}_{k-n+i}(S)\right),
\end{aligned}
$$

and the projection to the first summand is given by $\alpha \mapsto \alpha^{\prime}=\Gamma_{*} \alpha$.

We could also write down explicitly the projectors to the last $(n-m)$ summands; we omit the details here, as we will not need them. As before, by Manin's identity principle we have the following:

Corollary 3.10. If $X$ and $X^{\prime}$ are smooth and projective over some ground field $\mathbb{k}$, then there is an isomorphism of Chow motives over $\mathbb{k}$ :

$$
[\Gamma]^{t} \oplus\left(\bigoplus_{i=m+1}^{n} i_{*} \circ h^{n-i} \circ \psi^{*}\right): \mathfrak{h}\left(X^{\prime}\right) \oplus\left(\bigoplus_{i=m+1}^{n} \mathfrak{h}(S)(i)\right) \stackrel{\sim}{\rightarrow} \mathfrak{h}(X) .
$$

As before, the blowup formula for Chow motives of [39] could be viewed as the case $m=0$ of this corollary, as a blowup can be viewed as a standard flip of type $(n, 0)$.

\section{Main results}

Let $\mathscr{G}$ be a coherent sheaf of homological dimension $\leq 1$ on $X$ - that is, $X$ is covered by open subschemes $U \subset X$ over which there is a resolution $\mathscr{F} \stackrel{\sigma}{\longrightarrow} \mathscr{E} \rightarrow \mathscr{G}$ such that $\mathscr{F}$ and $\mathscr{E}$ are locally free of rank $m$ and $n$, respectively, and $\mathscr{G}=\operatorname{Coker}(\sigma)$ is of rank $r=n-m \geq 0$. Denote the projection by $\pi: \mathbb{P}(\mathscr{G}) \rightarrow X$. Similar to the projective bundle case, for any $i \in[0, r-1]$, denote by $\pi_{i}^{*}: \mathrm{CH}_{k-(r-1)+i}(X) \rightarrow \mathrm{CH}_{k}(\mathbb{P}(\mathscr{G}))$ the map $\pi_{i}^{*}\left(\_\right)=\zeta^{i} \cdot \pi^{*}\left(\_\right)$, where $\zeta=c_{1}\left(\mathscr{O}_{\mathbb{P}(\mathscr{G})}(1)\right)$. Consider the fiber product

$$
\Gamma:=\mathbb{P}(\mathscr{G}) \times_{X} \mathbb{P}\left(\mathscr{E} x t^{1}\left(\mathscr{G}, \mathscr{O}_{X}\right)\right)
$$

Denote the projections by $r_{+}: \Gamma \rightarrow \mathbb{P}(\mathscr{G})$ and $r_{-}: \Gamma \rightarrow \mathbb{P}\left(\mathscr{E} x t^{1}\left(\mathscr{G}, \mathscr{O}_{X}\right)\right)$. As before, we denote $\Gamma_{*}: \mathrm{CH}_{k-r}(\mathbb{P}(\mathscr{G})) \rightarrow \mathrm{CH}_{k}\left(\mathbb{P}\left(\mathscr{E} x t^{1}\left(\mathscr{G}, \mathscr{O}_{X}\right)\right)\right)$ and $\Gamma^{*}: \mathrm{CH}_{k}\left(\mathbb{P}\left(\mathscr{E} x t^{1}\left(\mathscr{G}, \mathscr{O}_{X}\right)\right)\right) \rightarrow$ $\mathrm{CH}_{k-r}(\mathbb{P}(\mathscr{G}))$ the maps induced by the correspondence $[\Gamma] \in \mathrm{CH}\left(\mathbb{P}(\mathscr{G}) \times \mathbb{P}\left(\mathscr{E} x t^{1}\left(\mathscr{G}_{,} \mathscr{O}_{X}\right)\right)\right)$ - that is,

$$
\Gamma_{*}\left(\_\right)=r_{-*} r_{+}^{*}\left(\_\right), \quad \Gamma^{*}\left(\_\right)=r_{+*} r_{-}^{*}\left(\_\right) .
$$

The main result of this paper is the following:

Theorem 4.1. Let $X$ be a Cohen-Macaulay scheme of pure dimension, and let $\mathscr{G}$ be a coherent sheaf of rank $r \geq 0$ on $X$ of homological dimension $\leq 1$. Assume either of the following: 
(A) $\mathbb{P}(\mathscr{G})$ and $\mathbb{P}\left(\mathscr{E} x t^{1}\left(\mathscr{G}, \mathscr{O}_{X}\right)\right)$ are nonsingular and quasi-projective, and we have $\operatorname{codim}\left(X^{\geq r+1}(\mathscr{G}) \subset X\right)=r+1, \quad \operatorname{codim}\left(X^{\geq r+i}(\mathscr{G}) \subset X\right) \geq r+2 i$ if $i \geq 2 ;$ or

(B) $\operatorname{codim}\left(X^{\geq r+i}(\mathscr{G}) \subset X\right)=i(r+i)$ (the expected codimension) for all $i \geq 1$.

Then for any $k \geq 0$, there is an isomorphism of Chow groups

$$
\bigoplus_{i=0}^{r-1} \mathrm{CH}_{k-(r-1)+i}(X) \oplus \mathrm{CH}_{k-r}\left(\mathbb{P}\left(\mathscr{E} x t^{1}\left(\mathscr{G}, \mathscr{O}_{X}\right)\right)\right) \stackrel{\sim}{\rightarrow} \mathrm{CH}_{k}(\mathbb{P}(\mathscr{G}))
$$

given by $\left(\oplus_{i=0}^{r-1} \alpha_{i}, \gamma\right) \mapsto \beta=\sum_{i=0}^{r-1} c_{1}\left(\mathscr{O}_{\mathbb{P}(\mathscr{G})}(1)\right)^{i} \cap \pi^{*} \alpha_{i}+\Gamma^{*} \gamma$. The projection $\beta \mapsto \alpha_{i}$ is given by the map $\pi_{i *}$ of Lemma 4.4, $0 \leq i \leq r-1$, and the projection $\beta \mapsto \gamma$ is given by $(-1)^{r} \Gamma_{*}$.

\section{Remark 4.2.}

(i) If $X$ is irreducible, then the dimension condition (4.1) of (A) is equivalent to the requirement that $\mathbb{P}\left(\mathscr{E} x t^{1}\left(\mathscr{G}, \mathscr{O}_{X}\right)\right)$ map birationally to $X^{\geq r+1}(\mathscr{G})$, and $\mathbb{P}(\mathscr{G})$, $\mathbb{P}\left(\mathscr{E} x t^{1}\left(\mathscr{G}, \mathscr{O}_{X}\right)\right)$, and $\Gamma$ are irreducible and have expected dimensions:

$$
\begin{gathered}
\operatorname{dim} \mathbb{P}(\mathscr{G})=\operatorname{dim} X-1+r, \quad \operatorname{dim} \mathbb{P}\left(\mathscr{E} x t^{1}\left(\mathscr{G}, \mathscr{O}_{X}\right)\right)=\operatorname{dim} X-1-r, \\
\operatorname{dim} \Gamma=\operatorname{dim} X-1 .
\end{gathered}
$$

(ii) The only place that we need $\mathbb{P}(\mathscr{G})$ and $\mathbb{P}\left(\mathscr{E} x t^{1}\left(\mathscr{G}, \mathscr{O}_{X}\right)\right)$ to be nonsingular and quasi-projective in (A) is in using Chow's moving lemma. Hence the result holds as long as Chow's moving lemma holds for $\mathbb{P}(\mathscr{G})$ and $\mathbb{P}\left(\mathscr{E} x t^{1}\left(\mathscr{G}, \mathscr{O}_{X}\right)\right)$.

(iii) It follows from [25, Theorem 3.4] that if $X$ is nonsingular and $\mathbb{P}(\mathscr{G})$ and $\mathbb{P}\left(\mathscr{E} x t^{1}\left(\mathscr{G}, \mathscr{O}_{X}\right)\right)$ have expected dimension, then $\mathbb{P}(\mathscr{G})$ is nonsingular if and only if $\mathbb{P}\left(\mathscr{E} x t^{1}\left(\mathscr{G}, \mathscr{O}_{X}\right)\right)$ is.

(iv) The required codimension $r+2 i$ when $i \geq 2$ in (A) is much weaker than the expected codimension $i(r+i)$, required by $(\mathrm{B})$, if $i \gg 1$. On the other hand, (B) requires only very weak regularity conditions on the schemes $-X$ being Cohen-Macaulay. (In fact, the Cohen-Macaulay condition can be dropped; we need each stratum $X^{\geq i}(\mathscr{G}) \backslash X^{\geq i+1}(\mathscr{G}) \subset X$ to be a regular immersion of expected dimension.)

Corollary 4.3. If $\mathbb{P}(\mathscr{G}), \mathbb{P}\left(\mathscr{E} x t^{1}\left(\mathscr{G}, \mathscr{O}_{X}\right)\right)$, and $X$ are smooth and projective over some ground field $\mathbb{k}$, then there is an isomorphism of Chow motives:

$$
\bigoplus_{i=0}^{r-1} h^{r-1-i} \circ \pi^{*} \oplus[\Gamma]^{t}: \bigoplus_{i=0}^{r-1} \mathfrak{h}(X)(i) \oplus \mathfrak{h}\left(\mathbb{P}\left(\mathscr{E} x t^{1}\left(\mathscr{G}, \mathscr{O}_{X}\right)\right)\right)(r) \stackrel{\sim}{\rightarrow} \mathfrak{h}(\mathbb{P}(\mathscr{G})) .
$$

Proof. Similarly as Corollary 3.4, for any smooth $T$ the same constructions and the theorem apply to $X \times T$ and $\mathscr{G} \otimes \mathscr{O}_{T}$; hence in particular the identities Id $=\Gamma^{*} \Gamma_{*}+$ $\sum \pi_{i}^{*} \pi_{i *}, \Gamma_{*} \Gamma^{*}=\mathrm{Id}, \pi_{i *} \pi_{i}^{*}=\mathrm{Id}$, and so on (see Lemmas 4.4 and 4.9) hold for all $X \times T$ and $\mathscr{G} \otimes \mathscr{O}_{T}$. Then the result follows from Manin's identity principle.

Before proceeding with the proofs of the theorem, we first explore some general facts. 


\section{Lemma 4.4.}

(1) Define $\pi_{i *}: \mathrm{CH}_{k}(\mathbb{P}(\mathscr{G})) \rightarrow \mathrm{CH}_{k-(r-1)+i}(X)$ the same way as in equation (2.1):

$$
\pi_{i *}(\ldots):=\sum_{j=0}^{r-1-i}(-1)^{j} c_{j}(\mathscr{G}) \cap \pi_{*}\left(\zeta^{r-1-i-j} \cdot\left(\_\right)\right), \quad \text { for } i=0,1, \ldots, r-1 .
$$

Then the maps $\pi_{i *}$ and $\pi_{j}^{*}$ satisfy

$$
\pi_{i *} \pi_{i}^{*}=\operatorname{Id}_{\mathrm{CH}(X)}, \quad \pi_{i *} \pi_{j}^{*}=0, \quad \text { if } i \neq j, i, j \in[0, r-1] .
$$

(2) In the local situation $\mathscr{G}=\operatorname{Coker}(\mathscr{F} \stackrel{\sigma}{\rightarrow} \mathscr{E})$, denote $q_{i *}$ the corresponding projection functor for the projective bundle $q: \mathbb{P}(\mathscr{E}) \rightarrow X$ defined by equation (2.1) and $\iota: \mathbb{P}(\mathscr{G}) \hookrightarrow \mathbb{P}(\mathscr{E})$ the natural inclusion; then the following holds:

$$
\pi_{i *}\left(\_\right)=\sum_{j=0}^{r-1-i}(-1)^{j} s_{j}(\mathscr{F}) \cdot q_{m+i+j *}\left(\iota_{*}\left(\_\right)\right) .
$$

If we consider the subgroup

$$
\mathrm{CH}_{k}(\mathbb{P}(\mathscr{G}))_{\text {tor. }}:=\left\{\beta \mid \pi_{i *} \beta=0, i \in[0, r-1]\right\} \subset \mathrm{CH}_{k}(\mathbb{P}(\mathscr{G})),
$$

then the lemma implies that there is a decomposition

$$
\mathrm{CH}_{k}(\mathbb{P}(\mathscr{G}))=\left(\bigoplus_{i=0}^{r-1} \pi_{i}^{*} \mathrm{CH}_{k-r-1+i}(X)\right) \oplus \mathrm{CH}_{k}(\mathbb{P}(\mathscr{G}))_{\text {tor. }}
$$

Proof. For simplicity, we may assume $\mathscr{G}=\operatorname{Coker}(\mathscr{F} \stackrel{\sigma}{\rightarrow} \mathscr{E})$; then $c(\mathscr{G})=c(\mathscr{E}) / c(\mathscr{F})$, and $\iota: \mathbb{P}(\mathscr{G}) \hookrightarrow \mathbb{P}(\mathscr{E})$ is given by a regular section of the vector bundle $\mathscr{F}^{\vee} \otimes \mathscr{O}_{\mathbb{P}(\mathscr{E})}(1)$. Then for any $a \in[0, r-1]$,

$$
\begin{aligned}
\pi_{a *}\left(\sum_{i=0}^{r-1} \pi_{i}^{*} \alpha_{i}\right) & =\pi_{a *}\left(\sum_{i=0}^{r-1} \zeta^{i} \cdot \iota^{*} q^{*} \alpha_{i}\right) \\
& =\sum_{j=0}^{r-1-a}(-1)^{j} c_{j}(\mathscr{G}) \cap q_{*} \iota_{*}\left(\zeta^{r-1-a-j} \cdot \sum_{i=0}^{r-1} \zeta^{i} \cdot \iota^{*} q^{*} \alpha_{i}\right) \\
& =\sum_{j=0}^{r-1-a}(-1)^{j} c_{j}(\mathscr{G}) \cap q_{*}\left(\sum_{i=0}^{r-1} c_{m}\left(\mathscr{F}^{\vee}(1)\right) \zeta^{r-1-a-j+i} c \cdot q^{*} \alpha_{i}\right) \\
& =\sum_{j=0}^{r-1-a} \sum_{i=0}^{r-1} \sum_{\nu=0}^{m}(-1)^{j+\nu} c_{j}(\mathscr{G}) c_{\nu}(\mathscr{F}) \cdot q_{*}\left(\zeta^{n-1-a-j+i-\nu} \cdot q^{*} \alpha_{i}\right)
\end{aligned}
$$


Now set $\mu:=\nu+j$, and notice that the terms in the foregoing which survive under $q_{*}$ have indices in the ranges $0 \leq \mu \leq i-1, i=\mu+a \geq a$, and $0 \leq \nu \leq \mu$ Therefore

$$
\begin{aligned}
\pi_{a *}\left(\sum_{i=0}^{r-1} \pi_{i}^{*} \alpha_{i}\right) & =\sum_{i=a}^{r-1} \sum_{\mu=0}^{i-a} \sum_{\nu=0}^{\mu}(-1)^{\mu} c_{j}(\mathscr{G}) c_{\nu}(\mathscr{F}) \cdot q_{*}\left(\zeta^{n-1+(i-a)-\mu} \cdot q^{*} \alpha_{i}\right) \\
& =\sum_{i=a}^{r-1} \sum_{\mu=0}^{i-a}(-1)^{\mu} c_{\mu}(\mathscr{E}) \cdot q_{*}\left(\zeta^{n-1+(i-a)-\mu} \cdot q^{*} \alpha_{i}\right) \\
& =\sum_{i=a}^{r-1} \sum_{\mu=0}^{i-a}(-1)^{\mu} c_{\mu}(\mathscr{E})(-1)^{i-a-\mu} s_{i-a-\mu}(\mathscr{E}) \cap \alpha_{i}=\alpha_{a} .
\end{aligned}
$$

Hence part (1) follows. In general, it suffices to notice that the maps $\pi_{i *} \pi_{i}^{*}$ and $\pi_{i *} \pi_{j}^{*}$ are globally defined and their values do not depend on local presentations.

Statement (2) follows directly from expressing $\iota_{*}\left(\sum_{i=0}^{r-1} \zeta^{i} \cdot \iota^{*} q^{*} \alpha_{i}\right)=c_{m}\left(\mathscr{F}^{\vee}(1)\right) \cap$ $\left(\sum_{i=0}^{r-1} \zeta^{i} \cdot q^{*} \alpha_{i}\right)$ in terms of the basis $\left\{\zeta^{i}\right\}$ of $\mathrm{CH}(\mathbb{P}(\mathscr{E}))$. Notice that one can also show part (2) first, and then part (1) follows easily.

For simplicity of notation, from now on we denote

$$
\mathscr{K}:=\mathscr{E} x t^{1}\left(\mathscr{G}, \mathscr{O}_{X}\right), \quad \pi^{\prime}: \mathbb{P}(\mathscr{K})=\mathbb{P}\left(\mathscr{E} x t^{1}\left(\mathscr{G}, \mathscr{O}_{X}\right)\right) \rightarrow X .
$$

Therefore we have a fibered diagram

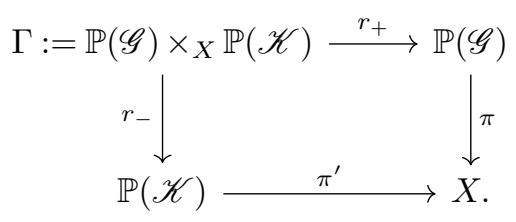

Lemma 4.5. Assume $\mathbb{P}(\mathscr{G}), \mathbb{P}(\mathscr{K})$, and $\Gamma$ have expected dimensions (see Remark 4.2i).

(1) The sheaf $\pi^{\prime * \mathscr{G}}$ has homological dimension $\leq 1$ and rank $r+1$ over $\mathbb{P}(\mathscr{K})$, and

$$
\Gamma=\mathbb{P}_{\mathbb{P}(\mathscr{K})}\left(\pi^{\prime * \mathscr{G}}\right)=\mathbb{P}_{\mathbb{P}(\mathscr{G})}\left(\pi^{*} \mathscr{K}\right) .
$$

(2) The excess bundle for diagram (4.3) is $\mathscr{O}(1,1):=\mathscr{O}_{\mathbb{P}(\mathscr{G})}(1) \otimes \mathscr{O}_{\mathbb{P}(\mathscr{K})}(1)$. Hence

$$
\pi^{*} \pi_{*}^{\prime}\left(\_\right)=r_{+*}\left(c_{1}(\mathscr{O}(1,1)) \cap r_{-}^{*}\left(\_\right)\right) .
$$

(3) The following holds:

$$
\pi_{i *} \pi_{j}^{*}=\delta_{i, j} \operatorname{Id}_{\mathrm{CH}(X)}, \quad \Gamma_{*} \pi_{i}^{*}=\pi_{i *} \Gamma^{*}=0, \quad \text { for all } i, j \in[0, r-1] .
$$

Proof. It suffices to prove in a local situation - that is, $0 \rightarrow \mathscr{F} \stackrel{\sigma}{\rightarrow} \mathscr{E} \rightarrow \mathscr{G} \rightarrow 0$ for vector bundles $\mathscr{F}$ and $\mathscr{E}$ of rank $m$ and $n$. Dually, we have $\mathscr{E} \vee \stackrel{\sigma^{\vee}}{\rightarrow} \mathscr{F} \vee \rightarrow \mathscr{K} \rightarrow 0$.

For part (1), notice that over $\mathbb{P}(\mathscr{K}) \subset \mathbb{P}(\mathscr{F} \vee)$, the composition of the map $\mathscr{O}_{\mathbb{P}(\mathscr{F} \vee}(-1) \rightarrow \pi^{\prime *} \mathscr{F} \stackrel{\sigma}{\rightarrow} \pi^{\prime *} \mathscr{E}$ is zero, hence $\sigma$ factorizes through a map of vector bundles 
$\bar{\sigma}: \mathcal{T}_{\mathbb{P}(\mathscr{F} \vee) / X}(-1) \rightarrow \pi^{\prime *} \mathscr{E}$. By reason of ranks, it easy to see that the following sequence is exact:

$$
\left.0 \rightarrow \mathcal{T}_{\mathbb{P}(\mathscr{F} \vee}\right) / X(-1) \stackrel{\bar{\sigma}}{\rightarrow} \pi^{* *} \mathscr{E} \rightarrow \pi^{\prime *} \mathscr{G} \rightarrow 0 .
$$

Therefore $\pi^{* * \mathscr{G}}$ has homological dimension $\leq 1$, and $\mathbb{P}\left(\pi^{* * \mathscr{G}}\right) \subset \mathbb{P}\left(\pi^{\prime * \mathscr{E}}\right)=\mathbb{P}(\mathscr{K}) \times_{X} \mathbb{P}(\mathscr{E})$ is the zero scheme of a regular section of the vector bundle $\Omega_{\mathbb{P}(\mathscr{F} \vee)}(1) \otimes \mathscr{O}_{\mathbb{P}(\mathscr{E})}(1)$. The last equality follows directly from commutativity of projectivization and fiber products.

For part (2), consider the following factorization of (the transpose of) diagram (4.3):

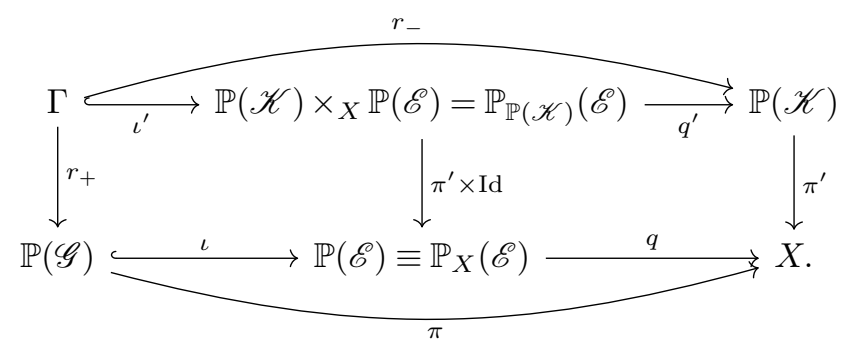

(Here for simplicity we use $q$ to denote both projections of projectivization of $\mathscr{E}$.) The normal bundles are $\mathscr{N}_{\iota}=\mathscr{F}^{\vee} \otimes \mathscr{O}_{\mathbb{P}(\mathscr{E})}(1)$ and $\mathscr{N}_{\iota^{\prime}}=\Omega_{\mathbb{P}(\mathscr{F} \vee}(1) \otimes \mathscr{O}_{\mathbb{P}(\mathscr{E})}(1)$. Since the right square of the diagram is a smooth and flat, the excess bundle is given by $r_{+}^{*} \mathscr{N}_{\iota} / \mathscr{N}_{\iota}=$ $\mathscr{O}(1,1)$.

For part (3), the first equality is Lemma 4.4. For any $\gamma \in \mathrm{CH}(\mathbb{P}(\mathscr{K}))$, for $i \in[0, r-1]$,

$$
\begin{aligned}
\pi_{i *} \Gamma^{*} \gamma & =\sum_{j=0}^{r-1-i} s_{j}\left(\mathscr{F}^{\vee}\right) \cdot q_{m+i+j *}\left(\iota_{*} r_{+*} r_{-}^{*} \gamma\right) \\
& =\sum_{j=0}^{r-1-i} s_{j}\left(\mathscr{F}^{\vee}\right) \cdot q_{m+i+j *}\left(\left(\pi^{\prime} \times \mathrm{Id}\right)_{*} \iota_{*}^{\prime} \iota^{\prime *}(\gamma \bigotimes 1)\right) \\
& =\sum_{j=0}^{r-1-i} s_{j}\left(\mathscr{F}^{\vee}\right) \cdot \pi_{*}^{\prime} q_{m+i+j *}\left(\iota_{*}^{\prime} \iota^{\prime *} q^{*} \gamma\right) \\
& \left.=\sum_{j=0}^{r-1-i} s_{j}\left(\mathscr{F}^{\vee}\right) \cdot \pi_{*}^{\prime} q_{m+i+j *}\left(c_{m-1}\left(\Omega_{\mathbb{P}(\mathscr{F} \vee}\right)(1) \otimes \mathscr{O}_{\mathbb{P}(\mathscr{E})}(1)\right) \cap q^{*} \gamma\right) \\
& =\sum_{j=0}^{r-1-i} s_{j}\left(\mathscr{F}^{\vee}\right) \cdot \pi_{*}^{\prime} q_{m+i+j *}\left(\left(\zeta^{m-1}+\text { lower-order terms }\right) \cdot q^{*} \gamma\right) \\
& =0 .
\end{aligned}
$$

(The last equality holds because $q_{m+i+j *}$ has index range $m+i+j \geq m$.) Similarly for any $\alpha \in \mathrm{CH}(X)$ and $i \in[0, r-1]$,

$$
\begin{aligned}
\Gamma_{*} \pi_{i}^{*} \alpha & =r_{-*} r_{+}^{*}\left(\zeta^{i} \cdot \pi^{*} \alpha\right)=r_{-*} \iota^{\prime *}\left(\zeta^{i} \cdot\left(\pi^{\prime} \times \mathrm{Id}\right)^{*} q^{*} \alpha\right)=q_{*} \iota_{*}^{\prime} \iota^{\prime *}\left(\zeta^{i} \cdot\left(\pi^{\prime *} \alpha \otimes 1\right)\right) \\
& =q_{*}\left(\left(\zeta^{m-1+i}+\text { lower-order terms }\right) \cdot q^{*} \pi^{\prime *} \alpha\right)=0,
\end{aligned}
$$

since $q$ is the projection of a $\mathbb{P}^{n-1}$-bundle and $m-1+i \leq m+r-2 \leq n-2$. 


\subsection{First approach}

In this approach, we use Chow's moving lemma, and hence need $\mathbb{P}(\mathscr{G})$ and $\mathbb{P}(\mathscr{K})$ to be nonsingular and quasi-projective. The idea is: over the open part of the first degeneracy locus, the theorem is almost the case of the Cayley trick. Then the 'error' terms over higher degeneracy loci can be estimated by dimension counting. A similar strategy was used by $\mathrm{Fu}$ and Wang to show the invariance of Chow groups under stratified Mukai flops [16].

We first need the following variant of the Cayley trick:

Lemma 4.6 (variant of the Cayley trick). Assume $\mathscr{G}$ is a coherent sheaf of homological dimension 1 over a variety $X$, and let $i: Z \hookrightarrow X$ be a locally complete intersection subscheme of codimension $r+1$, such that $\mathscr{G}$ has constant rank $r$ over $X \backslash Z$ and constant rank $r+1$ over $Z$. Denote $\Gamma:=\mathbb{P}_{Z}\left(i^{*} \mathscr{G}\right)=\mathbb{P}(\mathscr{G}) \times_{X} Z$, and denote $\Gamma_{*}: \mathrm{CH}(\mathbb{P}(\mathscr{G})) \rightarrow \mathrm{CH}(Z)$ and $\Gamma^{*}: \mathrm{CH}(Z) \rightarrow \mathrm{CH}(\mathbb{P}(\mathscr{G}))$ the maps induced by $[\Gamma]$ and $[\Gamma]^{t}$. Then the following hold:

$$
\Gamma_{*} \Gamma^{*}=(-1)^{r} \operatorname{Id}_{\mathrm{CH}(Z)}, \quad \pi_{i *} \pi_{j}^{*}=\delta_{i, j} \operatorname{Id}_{\mathrm{CH}(X)}, \quad \Gamma_{*} \pi_{i}^{*}=\pi_{i *} \Gamma^{*}=0,
$$

for any $i, j \in[0, r-1]$. Furthermore, the following decomposition of identity holds:

$$
\operatorname{Id}_{\mathrm{CH}(\mathbb{P}(\mathscr{G}))}=\Gamma^{*} \Gamma_{*}+\sum_{i=0}^{r-1} \pi_{i}^{*} \pi_{i *} .
$$

Proof. It suffices to notice that the argument of Theorem 3.1 for these statements depends only on the properties of the normal bundles, and thus still works here. More precisely, we may assume $\mathscr{G}=\operatorname{Coker}(\mathscr{F} \stackrel{\sigma}{\rightarrow} \mathscr{E})$ for simplicity; then over $Z$ there exists a line bundle $L$ such that there is an exact sequence of vector bundles

$$
\left.\left.0 \rightarrow L \rightarrow \mathscr{F}\right|_{Z} \rightarrow \mathscr{E}\right|_{Z} \rightarrow i^{*} \mathscr{G} \rightarrow 0
$$

Also we have a similar picture as with the Cayley trick (3.1):

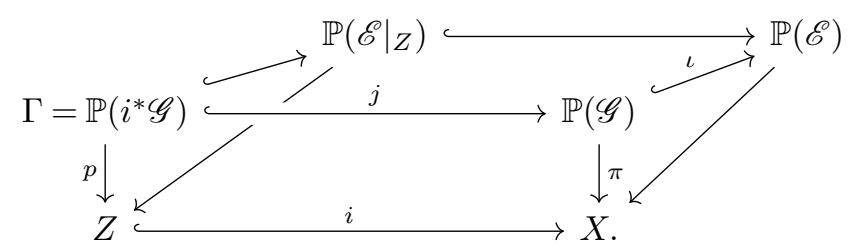

Denote $G_{Z}:=i^{*} \mathscr{G}$, which is a vector bundle on $Z$. Then it is easy to compute that the normal bundles are $\mathscr{N}_{i}=L^{\vee} \otimes G_{Z}, \mathscr{N}_{j}=L^{\vee} \otimes \Omega_{\mathbb{P}\left(G_{Z}\right) / Z}^{1}(1)$, and the excess bundle for the left square is $\mathscr{V}=p^{*} N_{i} / N_{j} \simeq L^{\vee} \otimes \mathscr{O}_{\mathbb{P}\left(G_{Z}\right)}$ (1) (see Lemma 4.10 for the more general situation). Therefore $\Gamma_{*} \Gamma^{*}=p_{*}\left(c_{r}\left(L^{\vee} \otimes \Omega_{\mathbb{P}\left(G_{Z}\right) / Z}^{1}(1)\right) \cap p^{*}\left(\_\right)\right)=p_{*}\left(\left((-1)^{r} \zeta^{r}+\right.\right.$ lower-order terms $\left.) \cap p^{*}\left(\_\right)\right)=(-1)^{r} \mathrm{Id}$, and the rest of the orthogonal relations follow from Lemma 4.5. Finally, for the last identity it suffices to show the surjectivity of $\Gamma^{*}+\sum \pi_{i}^{*}$. For any $\beta \in \mathrm{CH}\left(\mathbb{P}(\mathscr{G}), \beta^{\prime}=\beta-\sum \pi_{i}^{*} \pi_{i *} \beta\right.$ is supported on $\mathbb{P}_{Z}\left(G_{Z}\right)$, and hence can always be expressed in the form $\beta^{\prime}=j_{*}\left(p^{*} \beta_{0}+\left(\zeta-c_{1}(L)\right) \sum_{i=0}^{r-1} \zeta^{i} \cap p^{*} \beta_{i+1}\right)$. Therefore $\beta^{\prime}=$ $\Gamma^{*} \beta_{0}+\sum_{i=0}^{r-1} \pi_{i}^{*} i_{*} \beta_{i+1}^{\prime}$, and hence we are done. 
Remark 4.7. If we modify the map $f$ in Theorem 3.1 by $f: \oplus_{i=0}^{r-2} \gamma_{i} \mapsto$ $\left(-\oplus_{i=0}^{r-2} i_{*} \gamma_{i},\left(\zeta-c_{1}(L)\right) \sum_{i=0}^{r-2} \zeta^{i} \cdot p^{*} \gamma_{i}\right)$, then the sequence of the theorem is still exact. In fact, if we denote $p_{i *}$ the projectors with respect to $\mathscr{O}_{\mathbb{P}(\mathscr{E})}(1)$, then $p_{i}^{*} \circ i^{*}=j^{*} \circ \pi_{i}^{*}$ holds, although for $i \in[0, r-1], i_{*} \circ p_{i+1 *}$ and $\pi_{i *} \circ j_{*}$ are no longer the same, due to the additional factor $c_{1}(L)$ - but they differ by an invertible upper triangular change of basis as in Remark 2.6. Hence in Proposition 3.2, except for the key formula (a), which now becomes $\pi_{i}^{*} \circ i_{*}=j_{*}\left(\zeta-c_{1}(L) \cap p^{*}\left(\_\right)\right)$, the rest still holds. The process is similar for other the statements.

Denote $X_{i}:=X^{\geq r+i+1}(\mathscr{G})$ for $i \geq-1$; then there is a stratification $\cdots \subset X_{i+1} \subset X_{i} \subset$ $\cdots \subset X_{1} \subset X_{0} \subset X_{-1}=X$. This induces the corresponding stratifications $\mathbb{P}(\mathscr{G})_{i}:=\pi^{-1} X_{i}$, $\mathbb{P}(\mathscr{K})_{i}:=\pi^{\prime-1} X_{i}$, and $\Gamma_{i}:=r_{+}^{-1} \pi^{-1} X_{i}=r_{-}^{-1} \pi^{\prime-1} X_{i}$. Notice that $\mathbb{P}(\mathscr{G})_{-1}=\mathbb{P}(\mathscr{G})$, but

$$
\cdots \subset \mathbb{P}(\mathscr{K})_{1} \subset \mathbb{P}(\mathscr{K})_{0}=\mathbb{P}(\mathscr{K})_{-1}=\mathbb{P}(\mathscr{K}) \quad \text { and } \quad \cdots \subset \Gamma_{1} \subset \Gamma_{0}=\Gamma_{-1}=\Gamma,
$$

since $\mathbb{P}(\mathscr{K})$ is supported on $X_{0}$. Over each stratum $X_{i} \backslash X_{i+1}, i \geq 0$, diagram (4.3) is

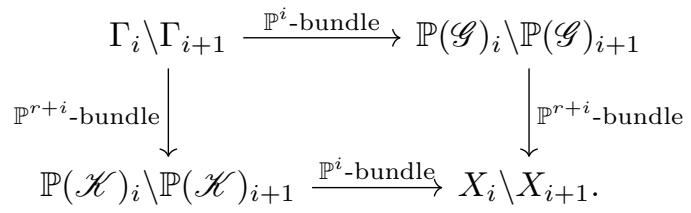

The codimension condition (4.1) translates into $\operatorname{dim} X_{0}=\operatorname{dim} X-(r+1)$ and $\operatorname{codim}\left(X_{i} \subset\right.$ $\left.X_{0}\right) \geq 2 i+1$. From the diagram, this implies that for any $i \geq 1$,

$$
\operatorname{codim}\left(\mathbb{P}(\mathscr{K})_{i} \subset \mathbb{P}(\mathscr{K})\right) \geq i+1 \quad \text { and } \quad \operatorname{codim}\left(\mathbb{P}(\mathscr{G})_{i} \subset \mathbb{P}(\mathscr{G})\right) \geq r+i+1 .
$$

Lemma 4.8. If $\mathbb{P}(\mathscr{K})=\mathbb{P}\left(\mathscr{E} x t^{1}\left(\mathscr{G}, \mathscr{O}_{X}\right)\right)$ is nonsingular and quasi-projective, and the dimension condition (4.1) holds, then the following holds:

$$
\Gamma_{*} \Gamma^{*}=(-1)^{r} \operatorname{Id}_{\mathbb{P}(\mathscr{K})} .
$$

Proof. The following arguments follow the strategy of Fu and Wang [16] for stratified Mukai flops, which is itself a generalization of [35]'s treatment for standard flops and flips (see also §3.2). For any class $[W] \in \mathrm{CH}_{k}(\mathbb{P}(\mathscr{K})$ ), by Chow's moving lemma we may assume that $W$ intersects transversely with $\sum_{i \geq 1} \mathbb{P}(\mathscr{K})_{i}$.

First, notice that over the open subset $\stackrel{\circ}{X}:=X \backslash X_{1}, \stackrel{\circ}{\mathbb{P}}(\mathscr{K}):=\mathbb{P}(\mathscr{K})_{0} \backslash \mathbb{P}(\mathscr{K})_{1} \simeq \stackrel{\circ}{Z}:=$ $X_{0} \backslash X_{1} \stackrel{i}{\rightarrow} \stackrel{\circ}{X}$ is an inclusion of codimension $r+1 ; \mathscr{G}$ has constant rank $r$ over $\stackrel{i}{X} \grave{Z}$ and constant rank $r+1$ over $\stackrel{\circ}{Z}$; and $\stackrel{\circ}{\Gamma} \simeq \mathbb{P}\left(i^{*} \mathscr{G}\right) \subset \stackrel{\circ}{\mathbb{P}}(\mathscr{G}):=\mathbb{P}(\mathscr{G})_{0} \backslash \mathbb{P}(\mathscr{G})_{1}$. Therefore we are in the situation of the variant of the Cayley trick (Lemma 4.6). Therefore if we set $\stackrel{\circ}{W}=W \cap \stackrel{\circ}{X}$, then the cycle $r_{+}^{*} r_{+*} r_{-}^{*}[\stackrel{\circ}{W}]$ is represented by a $k$-cycle $\stackrel{\circ}{W}$ which maps generically one-to-one to a $k$-cycle which is rationally equivalent to $\stackrel{\circ}{W}$, and $r_{*}[\stackrel{\circ}{W}]=$ $\Gamma_{*} \Gamma^{*}[\stackrel{\circ}{W}]=(-1)^{r}[\stackrel{\circ}{W}]$. 
Now back to the whole space. If we let $\widetilde{W}$ be the closure of $\stackrel{\circ}{W}$ in $\Gamma$, then

$$
r_{+}^{*} r_{+*} r_{-}^{*}[W]=[\widetilde{W}]+\sum_{C} a_{C}\left[F_{C}\right]
$$

where $\widetilde{W}$ is the $k$-dimensional cycle as before, mapping generically one-to-one to a $k$-cycle that is rationally equivalent to $(-1)^{r} W, a_{C} \in \mathbb{Z}$, and $F_{C}$ are $k$-dimensional irreducible schemes supported over $\pi^{\prime}\left(W \cap \sum_{i \geq 1} \mathbb{P}(\mathscr{K})_{i}\right)$. More precisely, let $C^{\prime}$ be irreducible components of $\pi^{-1} \pi^{\prime}\left(W \cap \sum_{i \geq 1} \mathbb{P}(\mathscr{K})_{i}\right)$; then the fiber $F_{C}$ runs through the components $\left\{C=\pi\left(C^{\prime}\right) \subset \pi^{\prime}\left(W \cap \sum_{i \geq 1} \mathbb{P}(\mathscr{K})_{i}\right)\right\}$; here different $C^{\prime}$ s may have the same image $C$. For any $F_{C}$, take the largest $i$ such that there is a component $D \subset \mathbb{P}(\mathscr{K})_{i}$ with $B_{C}:=\pi r_{+}\left(F_{C}\right)=\pi^{\prime} r_{-}\left(F_{C}\right) \subset \pi^{\prime}(W \cap D)$. For a general $s \in B_{C}$, the fiber $F_{C, s} \subset$ $\Gamma_{s} \simeq \mathbb{P}_{\kappa(s)}^{i} \times_{\kappa(s)} \mathbb{P}_{\kappa(s)}^{r+i}$ over $s$ has dimension

$$
\begin{aligned}
\operatorname{dim} F_{C, s} & \geq \operatorname{dim} F_{C}-\operatorname{dim}\left(B_{C}\right) \geq \operatorname{dim} F_{C}-\operatorname{dim} r_{-}\left(F_{C}\right) \\
& \geq \operatorname{dim} F_{C}-\operatorname{dim}(W \cap D)=k-\left(k-\operatorname{codim}\left(\mathbb{P}(\mathscr{K})_{i} \subset \mathbb{P}(\mathscr{K})\right)\right) \\
& =\operatorname{codim}\left(\mathbb{P}(\mathscr{K})_{i} \subset \mathbb{P}(\mathscr{K})\right) \geq i+1 .
\end{aligned}
$$

But since the general fiber of $\pi^{\prime}$ over $s$ has dimension $i$, then $F_{C, s}$ contains positivedimension fibers of $r_{-}$. Therefore $r_{-*}\left[F_{C}\right]=0$, and hence

$$
\Gamma_{*} \Gamma^{*}[W]=r_{-*}\left([\widetilde{W}]+\sum_{C} a_{C}\left[F_{C}\right]\right)=r_{-*}[\widetilde{W}]=(-1)^{r}[W] .
$$

Lemma 4.9. If $\mathbb{P}(\mathscr{G})$ is nonsingular and quasi-projective, and the dimension condition (4.1) holds, then for every $[V] \in \mathrm{CH}_{k}(\mathbb{P}(\mathscr{G}))_{\text {tor. }}$, the following holds:

$$
\Gamma^{*} \Gamma_{*}[V]=(-1)^{r}[V] .
$$

Proof. Set $[V] \in \mathrm{CH}_{k}(\mathbb{P}(\mathscr{G}))_{\text {tor. }}$ - that is, $[V] \in \mathrm{CH}_{k}(\mathbb{P}(\mathscr{G}))$ such that $\pi_{i *}[V]=0$ for all $i \in[0, r-1]$. By the moving lemma we may assume that $V$ intersects transversely with $\sum_{i \geq 1} \mathbb{P}(\mathscr{G})_{i}$. Similar to the proof of Lemma 4.8, by the variant of the Cayley trick (Lemma 4.6), $\Gamma^{*} \Gamma_{*}[\stackrel{\circ}{V}]=(-1)^{r}[\stackrel{\circ}{V}]$ over $\stackrel{\circ}{X}:=X \backslash X_{1}$, where $\stackrel{\circ}{V}=V \cap \stackrel{\circ}{X}$ and $\left[V_{0}\right] \in \mathbb{P}(\mathscr{G})_{\text {tor. }}$. Therefore there exists $\stackrel{\circ}{W}$ representing $\Gamma_{*}[\stackrel{\circ}{V}] \in \mathrm{CH}_{k-r}(\mathbb{P}(\mathscr{K}))$ such that $r_{-}^{-1}(\stackrel{\circ}{W})$ is a $k$-dimensional cycle and $r_{+*}\left(r_{-}^{-1}(\stackrel{\circ}{W})\right)$, though supported on $\mathbb{P}(\mathscr{G})_{1}$, is rationally equivalent to $(-1)^{r} \stackrel{\circ}{V}$ in $\mathbb{P}(\mathscr{G})$.

Therefore over the whole space, we have

$$
r_{-* *}^{*} r_{+}^{*}[V]=[\widetilde{V}]+\sum_{C} a_{C}\left[F_{C}\right]
$$

where $\widetilde{V}$ is the closure of $r_{-}^{-1}(\stackrel{\circ}{W})$ in $\Gamma$, and hence $r_{+*} \widetilde{V}$ is rationally equivalent to $(-1)^{r} V, a_{C} \in \mathbb{Z}$, and $F_{C}$ are irreducible $k$-dimensional cycles supported over 
$\pi\left(V \cap \sum_{i \geq 1} \mathbb{P}(\mathscr{G})_{i}\right)$. Similarly as before, for any $F_{C}$, take the largest $i \geq 1$ such that there is a component $D \subset \mathbb{P}(\mathscr{G})_{i}$ with $B_{C}:=\pi r_{+}\left(F_{C}\right)=\pi^{\prime} r_{-}\left(F_{C}\right) \subset \pi(V \cap D)$. For a general $s \in B_{C}$, the fiber $F_{C, s}$ has dimension

$$
\begin{aligned}
\operatorname{dim} F_{C, s} & \geq \operatorname{dim} F_{C}-\operatorname{dim}\left(B_{C}\right) \geq \operatorname{dim} F_{C}-\operatorname{dim} r_{-}\left(F_{C}\right) \\
& \geq \operatorname{dim} F_{C}-\operatorname{dim}(V \cap D)=\operatorname{codim}\left(\mathbb{P}(\mathscr{G})_{i} \subset \mathbb{P}(\mathscr{G})\right) \geq r+i+1
\end{aligned}
$$

Now since the general fiber of $\pi$ over $s$ has dimension $r+i$, then $F_{C, s}$ contains positivedimension fibers of $r_{+}$. Therefore $r_{+*}\left[F_{C}\right]=0$, and

$$
\Gamma^{*} \Gamma_{*}[V]=r_{+*}\left([\widetilde{V}]+\sum_{C} a_{C}\left[F_{C}\right]\right)=r_{+*}[\tilde{V}]=(-1)^{r}[V] .
$$

Proof of Theorem 4.1 under condition (A). The injectivity of map (4.2) follows directly from Lemmas 4.4 and 4.8; the surjectivity follows from Lemmas 4.4 and 4.9. This completes the proof.

\subsection{Second approach}

The idea of this second approach is that if we stratify the space $X$ as before, then over each stratum the theorem reduces to a situation very similar to the case of standard flips (§3.2). Since we will argue over each stratum, we will need all strata to achieve the expected dimensions, but we do not require regularity on the total space.

Lemma 4.10. Let $\mathscr{G}$ be a coherent sheaf on a Cohen-Macaulay scheme $X$ of homological dimension $\leq 1$ and rank $r$. For a fixed integer $i \geq 0$, denote $Z=X^{\geq r+i+1}(\mathscr{G})$, and assume $X^{\geq r+i+2}(\mathscr{G})=\emptyset$. (That is, $Z$ is the bottom degeneracy locus of $\mathscr{G}$, and $\mathscr{G}$ has constant rank $r+i+1$ over $Z$ and rank $\leq r+i$ over $X \backslash Z$.) Assume furthermore that $Z \subset X$ has the expected codimension $(i+1)(r+i+1)$. Denote $\mathscr{K}=\mathscr{E} x t^{1}(\mathscr{G}, \mathscr{O})$, with $i: Z \hookrightarrow X$ the inclusion. Then $G_{Z}:=i^{*} \mathscr{G}, K_{Z}:=i^{*} \mathscr{K}$ are vector bundles over $Z$ of rank $r+i+1$ and $i+1$, respectively. Set $\Gamma=\mathbb{P}(\mathscr{G}) \times_{X} \mathbb{P}(\mathscr{K})$ as usual, and assume that $\mathbb{P}(\mathscr{G}), \mathbb{P}(\mathscr{K})$, and $\Gamma$ have the expected dimensions (see Remark 4.2i). Consider the following base-change diagram for the fibered product $\Gamma=\mathbb{P}(\mathscr{G}) \times_{X} \mathbb{P}(\mathscr{K})$ along the base change $Z \hookrightarrow X$, with names of maps as indicated:

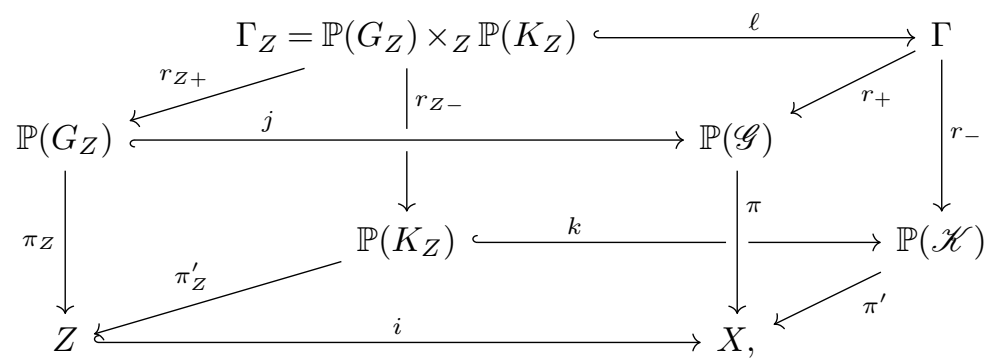

where $\Gamma_{Z}:=Z \times_{X} \Gamma=\mathbb{P}\left(G_{Z}\right) \times_{Z} \mathbb{P}\left(K_{Z}\right)$. 
Then the normal bundles of the closed immersions $i, j, k, \ell$ are respectively given by

$$
\begin{aligned}
\mathscr{N}_{i} & =G_{Z} \otimes K_{Z}, & \mathscr{N}_{j} & =\Omega_{\mathbb{P}\left(G_{Z}\right) / Z}(1) \otimes K_{Z}, \\
\mathscr{N}_{k} & =G_{Z} \otimes \Omega_{\mathbb{P}\left(K_{Z}\right) / Z}(1), & \mathscr{N}_{\ell} & =\Omega_{\mathbb{P}\left(G_{Z}\right) / Z}(1) \otimes \Omega_{\mathbb{P}\left(K_{Z}\right) / Z}(1) .
\end{aligned}
$$

The excess bundle for the front square is given by $\mathscr{V}=\mathscr{O}_{\mathbb{P}\left(G_{Z}\right)}(1) \otimes K_{Z}$, and the excess bundle for the back square is $\mathscr{V}^{\prime}=\mathscr{O}_{\mathbb{P}\left(G_{Z}\right)}(1) \otimes \Omega_{\mathbb{P}\left(K_{Z}\right) / Z}(1)$. Therefore

$$
\pi^{*} i_{*}\left(\_\right)=j_{*}\left(c_{t o p}(\mathscr{V}) \cap \pi_{Z}^{*}\left(\_\right)\right), \quad r_{-}^{*} k_{*}\left(\_\right)=\ell_{*}\left(c_{t o p}\left(\mathscr{V}^{\prime}\right) \cap r_{Z-}^{*}\left(\_\right)\right) .
$$

Similarly, the excess bundle for the bottom square is given by $\mathscr{W}=G_{Z} \otimes \mathscr{O}_{\mathbb{P}\left(K_{Z}\right)}(1)$, and for the top square it is $\mathscr{W}^{\prime}=\Omega_{\mathbb{P}\left(G_{Z}\right) / Z}(1) \otimes \mathscr{O}_{\mathbb{P}\left(K_{Z}\right)}(1)$. Therefore

$$
\pi^{\prime *} i_{*}\left(\_\right)=k_{*}\left(c_{t o p}(\mathscr{W}) \cap \pi_{Z}^{\prime *}\left(\_\right)\right), \quad r_{+}^{*} j_{*}\left(\_\right)=\ell_{*}\left(c_{t o p}\left(\mathscr{W}^{\prime}\right) \cap r_{Z+}^{*}\left(\_\right)\right) .
$$

Proof. As the statements are local, we may assume $\mathscr{G}=\operatorname{Coker}(F \stackrel{\sigma}{\rightarrow} E)$, where $E, F$ are vector bundles of rank $n$ and $m$. Then by our assumption on $Z$ and Lemma 2.3, $Z \subset X$ is a closed locally complete intersection subscheme, and $\mathscr{N}_{i}=G_{Z} \otimes K_{Z}$. Moreover, the image $\left.\operatorname{im}\left(\left.\sigma\right|_{Z}\right) \subset E\right|_{Z}$ is a vector sub-bundle; let us denote it by $B_{Z}$. Therefore the map $\left.\sigma\right|_{Z}$ induces two short exact sequences of vector bundles over $Z$ :

$$
\left.0 \rightarrow K_{Z}^{\vee} \rightarrow F\right|_{Z} \rightarrow B_{Z} \rightarrow 0,\left.\quad 0 \rightarrow B_{Z} \rightarrow E\right|_{Z} \rightarrow G_{Z} \rightarrow 0 .
$$

Next, over $\mathbb{P}(\mathscr{G}) \subset \mathbb{P}(E)$, the composition $\pi^{*} F \stackrel{\pi^{*} \sigma}{\longrightarrow} \pi^{*} E \rightarrow \mathscr{O}_{\mathbb{P}(E)}(1)$ is zero, hence $\pi^{*} \sigma$ factors through a map between vector bundles $\widetilde{\sigma}: \pi^{*} F \rightarrow \Omega_{\mathbb{P}(E) / X}^{1}(1)$. The rank of $\widetilde{\sigma}$ at a point $p \in \mathbb{P}(\mathscr{G})$ agrees with the rank of $\sigma$ at $\pi(p)$, so $\widetilde{Z}:=\pi^{-1}(Z)=\mathbb{P}\left(G_{Z}\right)$ is the bottom degeneracy locus of $\widetilde{\sigma}$. We claim that there is an exact sequence of vector bundles

$$
\left.0 \rightarrow \pi_{Z}^{*} K_{Z}^{\vee} \rightarrow \pi_{Z}^{*} F_{Z} \stackrel{\widetilde{\sigma}}{\rightarrow} \Omega_{\mathbb{P}(E) / X}^{1}(1)\right|_{\widetilde{Z}} \rightarrow \Omega_{\mathbb{P}\left(G_{Z}\right) / Z}^{1}(1) \rightarrow 0 .
$$

Then by Lemma $2.3, \mathscr{N}_{j}=\Omega_{\mathbb{P}\left(G_{Z}\right) / Z}^{1}(1) \otimes K_{Z}$ and $\mathscr{V}=\pi_{Z}^{*} \mathscr{N}_{i} / \mathscr{N}_{j} \simeq \mathscr{O}_{\mathbb{P}\left(G_{Z}\right)}(1) \otimes K_{Z}$.

To prove the claim, it suffices to notice that over $\widetilde{Z}$ there is a commutative diagram

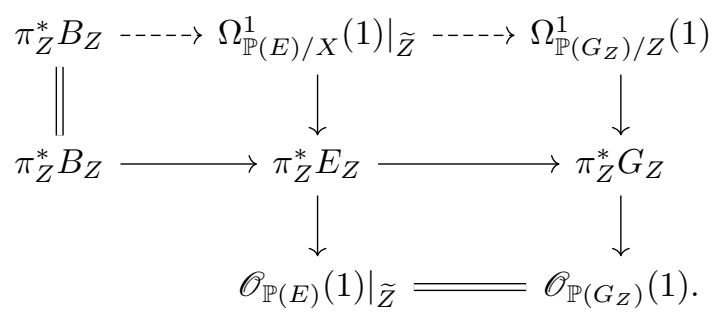

The three columns and the last two rows are exact, hence the first row is a short exact sequence. Combining with the short exact sequence of vector bundles $0 \rightarrow \pi_{Z}^{*}\left(K_{Z}^{\vee}\right) \rightarrow$ $\pi_{Z}^{*}\left(\left.F\right|_{Z}\right) \rightarrow \pi_{Z}^{*}\left(B_{Z}\right) \rightarrow 0$, the claim follows. Notice that we do not use the condition $n \geq m$, so the same argument works for all the other cases. 
Lemma 4.11 ('virtual' flips). In the situation of Lemma 4.10, denote

$$
\begin{aligned}
& \Psi_{*}\left(\_\right):=r_{Z-*}\left(c_{t o p}\left(\mathscr{W}^{\prime}\right) \cap r_{Z+}^{*}\left(\_\right)\right): \mathrm{CH}\left(\mathbb{P}\left(G_{Z}\right)\right) \rightarrow \mathrm{CH}\left(\mathbb{P}\left(K_{Z}\right)\right), \\
& \Psi^{*}\left(\_\right):=r_{Z+*}\left(c_{t o p}\left(\mathscr{V}^{\prime}\right) \cap r_{Z-}^{*}\left(\_\right)\right): \mathrm{CH}\left(\mathbb{P}\left(K_{Z}\right)\right) \rightarrow \mathrm{CH}\left(\mathbb{P}\left(G_{Z}\right)\right) .
\end{aligned}
$$

Furthermore, for any $a \in[0, r-1]$, denote $\pi_{Z, a}^{*}\left(\_\right):=c_{t o p}(\mathscr{V}) \cdot \zeta^{a} \cap \pi_{Z}^{*}\left(\_\right)$. Then the following are true:

(1) $\Psi_{*} \Psi^{*}=(-1)^{r}$ Id.

(2) For any $k \geq 0$, there is an isomorphism of Chow groups

$$
\bigoplus_{a=0}^{r-1} \mathrm{CH}_{k-(r-1)+a}(Z) \oplus \mathrm{CH}_{k-r}\left(\mathbb{P}\left(K_{Z}\right)\right) \stackrel{\sim}{\rightarrow} \mathrm{CH}_{k}\left(\mathbb{P}\left(G_{Z}\right)\right),
$$

given by $\left(\oplus_{a=0}^{r-1} \alpha_{a}, \gamma\right) \mapsto \sum_{a=0}^{r-1} \pi_{Z, a}^{*} \alpha_{a}+\Psi^{*} \gamma$.

(3) For any $a \in[0, r-1]$,

$$
\Gamma^{*} k_{*}\left(\_\right)=j_{*} \Psi^{*}\left(\_\right), \quad \Gamma_{*} j_{*}\left(\_\right)=k_{*} \Psi_{*}\left(\_\right), \quad \pi_{a}^{*} i_{*}\left(\_\right)=j_{*} \pi_{Z, a}^{*}\left(\_\right) .
$$

Proof. For the first two statements, notice that if we write $F=G_{Z}^{\vee}, F^{\prime}=K_{Z}^{\vee}$, with rank $n=r+i$ and $m=i$, and $S=Z$, then $P=\mathbb{P}\left(G_{Z}\right), P^{\prime}=\mathbb{P}\left(K_{Z}\right)$, and $E=\Gamma_{Z}$, and we are in a very similar situation as the case of standard flips (§3.2). In fact, for part (1), using the notation of the proof of Theorem 3.6, $\Psi_{*}$ and $\Psi^{*}$ correspond to the correspondences given by $(-1)^{n} c_{n}\left(\mathscr{V}^{\prime}\right)$ and $(-1)^{m} c_{m}(\mathscr{V})$, respectively (instead of $c_{m}(\mathscr{V})$ for $\Phi_{*}$ and $c_{n}\left(\mathscr{V}^{\prime}\right)$ for $\left.\Phi^{*}\right)$. However, by the commutativity of the intersection product, the composition $c_{n}\left(\mathscr{V}^{\prime}\right) * c_{m}(\mathscr{V})$ is still computed by the same formula as $c_{m}(\mathscr{V}) * c_{n}\left(\mathscr{V}^{\prime}\right)$, with the role of the first and third factors of the product $P^{\prime} \times{ }_{S} P \times{ }_{S} P^{\prime}$ switched. Hence $c_{n}\left(\mathscr{V}^{\prime}\right) * c_{m}(\mathscr{V})=$ $\left[\Delta_{P^{\prime}}\right]$, and $\Psi_{*} \Psi^{*}=(-1)^{m+n} \mathrm{Id}=(-1)^{r} \mathrm{Id}$.

For part (2), the same argument as in Lemma 3.8 works. In fact, the image of $\Psi^{*}$ is the 'sub-CH( $Z$-module' generated by $1, \zeta, \ldots, \zeta^{i}$. Hence up to elements of $\operatorname{Im} \Psi^{*}=$ $\operatorname{Span}\left\{1, \zeta^{1}, \ldots, \zeta^{i}\right\}$, the map

$$
\pi_{Z, a}^{*}\left(\_\right)=\zeta^{a} \cdot c_{\mathrm{top}}(\mathscr{V}) \cdot \cap \pi_{Z}^{*}\left(\_\right)=\zeta^{a} \cdot\left(\zeta^{i+1}+\text { lower-order terms }\right) \cdot \pi_{Z}^{*}\left(\_\right)
$$

hits each element of the basis $\left\{\zeta^{i+a+1} \bmod \operatorname{Im} \Psi^{*}\right\}_{a \in[0, r-1]}$ of the quotient $\operatorname{CH}\left(\mathbb{P}\left(G_{Z}\right)\right) /$ $\operatorname{Im} \Psi^{*}$. Therefore the result follows.

For part (3), it follows directly from Lemma 4.10 that for any $\gamma \in \mathrm{CH}\left(\mathbb{P}\left(K_{Z}\right)\right)$,

$$
\Gamma^{*} k_{*} \gamma=r_{+*} r_{-}^{*} k_{*} \gamma=r_{+*} \ell_{*}\left(c_{\mathrm{top}}\left(\mathscr{V}^{\prime}\right) \cap r_{Z-}^{*} \gamma\right)=j_{*} r_{Z+*}\left(c_{\mathrm{top}}\left(\mathscr{V}^{\prime}\right) \cap r_{Z-}^{*} \gamma\right)=j_{*} \Psi^{*} \gamma,
$$

and similarly, $\Gamma_{*} j_{*}=k_{*} \Psi_{*}$. Also, for any $a \in[0, r-1]$ and $\alpha \in \mathrm{CH}(Z)$,

$$
\pi_{a}^{*}\left(i_{*} \alpha\right)=\pi^{*}\left(\zeta^{a} \cdot i_{*} \alpha\right)=j_{*}\left(\zeta^{a} \cdot c_{\mathrm{top}}(\mathscr{V}) \cap \pi_{Z}^{*}(\alpha)\right)=j_{*} \pi_{Z, a}^{*}(\alpha) .
$$

Proof of Theorem 4.1 under condition (B). Stratify the space $X$ the same way as in the first approach, namely $X_{i}:=X^{\geq r+i+1}(\mathscr{G})$ for $i \geq-1$, and similarly for $\mathbb{P}(\mathscr{G})_{i}, \mathbb{P}(\mathscr{K})_{i}$, and $\Gamma_{i}$. For each $i \geq-1$, we will denote the natural inclusions by $i_{i}: X_{i} \hookrightarrow X, j_{i}: \mathbb{P}(\mathscr{G})_{i} \hookrightarrow$ $\mathbb{P}(\mathscr{G}), k_{i}: \mathbb{P}(\mathscr{K})_{i} \hookrightarrow \mathbb{P}(\mathscr{K})$, and $\ell_{i}: \Gamma_{i} \hookrightarrow \Gamma$. For $i \geq 0$, we also denote $i_{i, i-1}: X_{i} \hookrightarrow X_{i-1}$ 
the natural inclusion, and $j_{i, i-1}, k_{i, i-1}$, and $\ell_{i, i-1}$ are defined similarly. Finally, for each pair $(i, j)$ with $j>i \geq-1$, denote $X_{i \backslash j}:=X_{i} \backslash X_{j} ; \mathbb{P}(\mathscr{G})_{i \backslash j}, \mathbb{P}(\mathscr{K})_{i \backslash j}$, and $\Gamma_{i \backslash j}$ are defined in the same manner. By abuse of notation, the inclusion $i_{i}: X_{i \backslash j} \hookrightarrow X \backslash X_{j}=X_{-1 \backslash j}$ is also denoted by $i_{i}$, and similarly for other inclusions.

For any fixed integer $i \geq 0$, if we assume that condition (B) of Theorem 4.1 is satisfied, then $Z:=X_{i \backslash i+1} \subset X \backslash X_{i+1}=X_{-1 \backslash i+1}$ is a locally complete intersection subscheme of codimension $(i+1)(r+i+1)$, and $\mathscr{G}$ has constant rank $r+i+1$ over $Z$. Therefore the conditions of Lemma 4.10 are satisfied by $Z \subset X \backslash X_{i+1}$ and $\mathscr{G}$, with $\mathbb{P}\left(G_{Z}\right)=\mathbb{P}(\mathscr{G})_{i \backslash i+1}$, $\mathbb{P}\left(K_{Z}\right)=\mathbb{P}(\mathscr{K})_{i \backslash i+1}$, and $\Gamma_{Z}=\Gamma_{i \backslash i+1}$, as well as $i=i_{i}, j=j_{i}, k=k_{i}$, and $\ell=\ell_{i}$. Hence the results of Lemma 4.11 can be applied.

Now our goal is to show that the isomorphism of Lemma 4.11(2) over each stratum can indeed be integrated into an isomorphism of map (4.2) of Theorem 4.1.

Surjectivity of map (4.2). For each $i \geq-1$, there is an exact sequence

$$
\mathrm{CH}\left(\mathbb{P}(\mathscr{G})_{i \backslash i+1}\right) \stackrel{j_{i *}}{\longrightarrow} \mathrm{CH}\left(\mathbb{P}(\mathscr{G}) \backslash \mathbb{P}(\mathscr{G})_{i+1}\right) \longrightarrow \mathrm{CH}\left(\mathbb{P}(\mathscr{G}) \backslash \mathbb{P}(\mathscr{G})_{i}\right) \longrightarrow 0,
$$

for which if $i=i_{\max }+1$, then the middle term is the whole space, where $i_{\max }$ is the largest number such that $X_{i_{\max }} \neq \emptyset$. (Since $X$ is locally Noetherian of pure dimension, there exist only finitely many strata, and such an $i_{\max }$ always exists.) Therefore inductively we see that $\mathrm{CH}(\mathbb{P}(\mathscr{G}))$ is generated by the images of $j_{i *}: \mathrm{CH}\left(\mathbb{P}(\mathscr{G})_{i \backslash i+1}\right) \rightarrow \mathrm{CH}(\mathbb{P}(\mathscr{G}))$ for all strata $\mathbb{P}(\mathscr{G})_{i \backslash i+1}, i \geq-1$, where $i=-1$ corresponds to the open stratum.

Hence we need only show that the image of map (4.2) contains the image of the strata $\mathrm{CH}\left(\mathbb{P}(\mathscr{G})_{i \backslash i+1}\right)$ in $\mathrm{CH}(\mathbb{P}(\mathscr{G}))$ for each $i \geq-1$. The open-stratum case $i=-1$ follows from the projective bundle formula. For other cases - that is, $i \geq 0-\operatorname{set} Z:=X_{i \backslash i+1} \subset$ $X \backslash X_{i+1}$ as before, and for simplicity denote $j_{*}:=j_{i *}: \mathrm{CH}\left(\mathbb{P}(\mathscr{G})_{i \backslash i+1}\right) \rightarrow \mathrm{CH}(\mathbb{P}(\mathscr{G}))$, which agrees with the notation of Lemmas 4.10 and 4.11 , and similarly for the maps $i, k, \ell$. Then by Lemma $4.11(2)$, any $\alpha \in \mathrm{CH}\left(\mathbb{P}(\mathscr{G})_{i \backslash i+1}\right)=\mathrm{CH}\left(\mathbb{P}\left(G_{Z}\right)\right)$ can be written as $\alpha=\sum_{a=0}^{r-1} \pi_{Z, a}^{*} \alpha_{a}+\Psi^{*} \gamma$, for certain $\alpha_{a} \in \mathrm{CH}(Z)$ and $\gamma \in \mathbb{P}\left(K_{Z}\right)=\mathbb{P}(\mathscr{K})_{i \backslash i+1}$. Therefore by Lemma $4.11(3)$,

$$
j_{*}(\alpha)=j_{*}\left(\sum_{a=0}^{r-1} \pi_{Z, a}^{*} \alpha_{a}+\Psi^{*} \gamma\right)=\sum_{a=0}^{r-1} \pi_{a}^{*}\left(i_{*} \alpha_{a}\right)+\Gamma^{*}\left(k_{*} \gamma\right)
$$

That is, the image of $j_{*}$ is contained in the image of map (4.2), and hence we are done.

Injectivity of map (4.2). This part is a little tricky; the key observation is that the excision exact sequence becomes a short exact sequence if we take the image of first map. The injectivity of $\pi_{a}^{*}$ follows from Lemma 4.4; it remains to show the injectivity of $\Gamma^{*}$. For each $i \geq-1$, there is a commutative diagram of short exact sequences

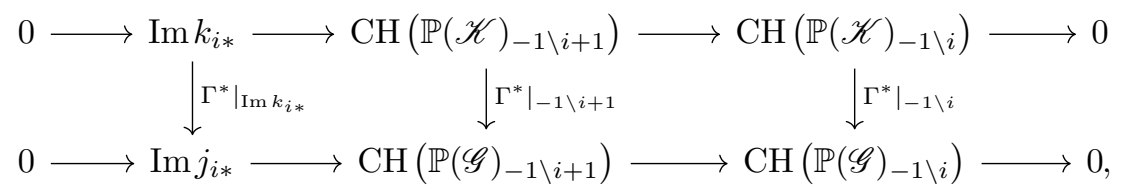


where we recall that the maps $k_{i *}$ and $j_{i *}$ are the inclusions to (an open subset of) the whole space:

$$
k_{i *}: \mathrm{CH}\left(\mathbb{P}(\mathscr{K})_{i \backslash i+1}\right) \rightarrow \mathrm{CH}\left(\mathbb{P}(\mathscr{K})_{-1 \backslash i}\right), \quad j_{i *}: \mathrm{CH}\left(\mathbb{P}(\mathscr{G})_{i \backslash i+1}\right) \rightarrow \mathrm{CH}\left(\mathbb{P}(\mathscr{G})_{-1 \backslash i+1}\right) .
$$

We want to show that for each $i \geq 0$, the map $\Gamma^{*} \mid \operatorname{Im} k_{i *}$ is injective. Set $Z:=X_{i \backslash i+1} \subset$ $X \backslash X_{i+1}$ as before; then the question reduces to showing that in the commutative diagram

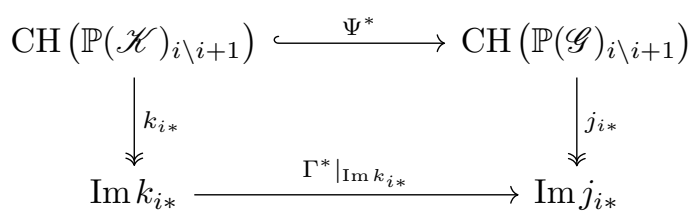

(which is commutative by Lemma 4.11(3)), the injection $\Psi^{*}$ induces an injection $\Gamma^{*}$ on the image. In fact, for any $\gamma \in \mathrm{CH}\left(\mathbb{P}(\mathscr{K})_{i \backslash i+1}\right)$, if $\Gamma^{*} k_{i *} \gamma=j_{i *} \Psi^{*} \gamma=0$, then by Lemma 4.11(1), we have $\gamma=(-1)^{r} \Psi_{*} \Psi^{*} \gamma$. Therefore by Lemma 4.11(3),

$$
k_{i *} \gamma=(-1)^{r} k_{i *} \Psi_{*} \Psi^{*} \gamma=(-1)^{r} \Gamma_{*} j_{i *} \Psi^{*} \gamma=0 .
$$

Hence $\left.\Gamma^{*}\right|_{\operatorname{Im} k_{i *}}$ is injective. Now by induction, starting with the case $i=0$, when the injectivity of $\left.\Gamma^{*}\right|_{-1 \backslash 1}$ follows from the commutative diagram

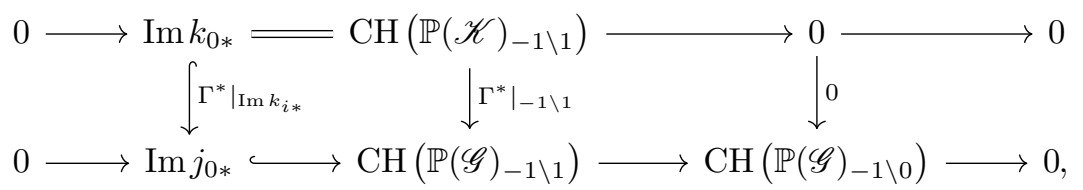

we can inductively show that $\left.\Gamma^{*}\right|_{-1 \backslash i}$ is injective for all $i=0,1,2, \ldots, i_{\max }, i_{\max }+1$, where $i_{\max }$ is the largest number such that $X_{i_{\max }} \neq \emptyset$. Therefore $\Gamma^{*}=\left.\Gamma^{*}\right|_{-1 \backslash i_{\max }+1}$ is injective on the whole space. Notice that from the preceding argument, we also obtain $\Gamma_{*} \Gamma^{*}=(-1)^{r} \operatorname{Id}$, since it is true on the image of each stratum. Together with Lemmas 4.4 and 4.5 , this completes the proof of Theorem 4.1 .

\subsection{First examples}

4.3.1. Universal Hom spaces. Let $S$ be a Cohen-Macaulay scheme, and let $V$ and $W$ be two vector bundles over $S$. Without loss of generality, we may assume $\operatorname{rank} W \leq \operatorname{rank} V$. Consider the total space of maps between $V$ and $W$ :

$$
X=\left|\mathscr{H}_{m_{S}}(W, V)\right|=\left|\mathscr{H}_{m_{S}}\left(V^{\vee}, W^{\vee}\right)\right| .
$$

Then there are tautological maps over $X$ :

$$
\phi: W \otimes \mathscr{O}_{X} \rightarrow V \otimes \mathscr{O}_{X}, \quad \phi^{\vee}: V^{\vee} \otimes \mathscr{O}_{X} \rightarrow W^{\vee} \otimes \mathscr{O}_{X}
$$

Let $\mathscr{G}=\operatorname{Coker}(\phi)$ and $\mathscr{K}=\mathscr{E} x t^{1}\left(\mathscr{G}, \mathscr{O}_{X}\right)=\operatorname{Coker}\left(\phi^{\vee}\right)$. Then it is easy to see that condition (B) of Theorem 4.1 is satisfied, and Theorem 4.1 holds for

$$
\mathbb{P}(\mathscr{G})=\operatorname{Tot}_{\mathbb{P}(V)}\left(W^{\vee} \otimes_{S} \Omega_{\mathbb{P}(V) / S}(1)\right), \quad \mathbb{P}(\mathscr{K})=\operatorname{Tot}_{\mathbb{P}\left(W^{\vee}\right)}\left(\Omega_{\mathbb{P}\left(W^{\vee}\right) / S}(1) \otimes_{S} V\right) .
$$


Notice that any map $\sigma: W \rightarrow V$ over $S$ determines a section $s_{\sigma}: S \rightarrow X$ such that $s_{\sigma}^{*} \phi=\sigma$ and $s_{\sigma}^{*} \phi^{\vee}=\sigma^{\vee}$. Then $\operatorname{Coker}(\sigma)$ and $\operatorname{Coker}\left(\sigma^{\vee}\right)$ (and their projectivizations) are just the pullbacks of $\mathscr{G}$ and $\mathscr{K}$ (and the projectivizations $\mathbb{P}(\mathscr{G})$ and $\mathbb{P}(\mathscr{K})$ ) along the section $\operatorname{map} s_{\sigma}$.

Similarly, we can consider the projectivization version

$$
Y=\mathbb{P}_{S, \operatorname{sub}}\left(\mathscr{H}_{0 m_{S}}(W, V)\right)=\mathbb{P}_{S, \operatorname{sub}}\left(\mathscr{H}_{m_{S}}\left(V^{\vee}, W^{\vee}\right)\right) .
$$

Over $Y$ there are tautological maps

$$
\psi: W \otimes \mathscr{O}_{Y}(-1) \rightarrow V \otimes \mathscr{O}_{Y}, \quad \psi^{\vee}: V^{\vee} \otimes \mathscr{O}_{Y} \rightarrow W^{\vee} \otimes \mathscr{O}_{Y}(1)
$$

Then condition (B) of Theorem 4.1 is satisfied for $\mathscr{M}=\operatorname{Coker}(\psi)$ and $\mathscr{N}=$ $\mathscr{E} x t^{1}\left(\mathscr{M}, \mathscr{O}_{X}\right)=$ Coker $\left(\psi^{\vee}\right)$, and Theorem 4.1 holds for

$$
\mathbb{P}(\mathscr{M})=\mathbb{P}_{\mathbb{P}(V), \text { sub }}\left(W^{\vee} \otimes \Omega_{\mathbb{P}(V) / S}(1)\right), \quad \mathbb{P}(\mathscr{N})=\mathbb{P}_{\mathbb{P}\left(W^{\vee}\right), \text { sub }}\left(\Omega_{\mathbb{P}\left(W^{\vee}\right) / S}(1) \otimes V\right) .
$$

One may also consider the linear sections of the space $Y$ as in HPD theory $[6,30]$.

4.3.2. Flops and Springer resolutions. In the situation of Theorem 4.1, if we take $r=0$, then $\mathbb{P}(\mathscr{G})$ and $\mathbb{P}(\mathscr{K})=\mathbb{P}\left(\mathscr{E} x t^{1}\left(\mathscr{G}, \mathscr{O}_{X}\right)\right)$ are both Springer-type partial desingularizations of the first degeneracy locus $X_{\mathrm{sg}}(\mathscr{G})=X^{\geq 1}(\mathscr{G}) \subset X$. They are related by a flop, and $\Gamma=\mathbb{P}(\mathscr{G}) \times_{X} \mathbb{P}(\mathscr{K})$ is the graph closure for the rational mapP $(\mathscr{G}) \rightarrow \mathbb{P}(\mathscr{K})$. For simplicity, we assume $X$ is irreducible. Then Theorem 4.1 states that if either

(A) $\mathbb{P}(\mathscr{G})$ and $\mathbb{P}(\mathscr{K})$ are smooth and quasi-projective (hence both resolutions of $\left.X_{\mathrm{sg}}(\mathscr{G})\right), \Gamma=\mathbb{P}(\mathscr{G}) \times_{X} \mathbb{P}(\mathscr{K})$ is irreducible, and $\operatorname{dim} \Gamma=\operatorname{dim} X-1$, or

(B) $X$ is Cohen-Macaulay and $\operatorname{codim} X^{\geq i}(\mathscr{G})=i^{2}$ for $i \geq 1$,

then the graph closure $\Gamma$ of the flop $\mathbb{P}(\mathscr{G}) \rightarrow \mathbb{P}(\mathscr{K})$ induces isomorphisms

$$
\Gamma^{*}: \mathrm{CH}(\mathbb{P}(\mathscr{K})) \simeq \mathrm{CH}(\mathbb{P}(\mathscr{G})), \quad \Gamma_{*}: \mathrm{CH}(\mathbb{P}(\mathscr{G})) \simeq \mathrm{CH}(\mathbb{P}(\mathscr{K})) .
$$

4.3.3. Cohen-Macaulay subschemes of codimension 2. Let $X$ be an irreducible scheme and $Z \subset X$ a codimension 2 subscheme whose ideal $\mathscr{I}_{Z}$ has homological dimension $\leq 1$. This holds in particular for any codimension 2 Cohen-Macaulay subscheme $Z \subset X$ inside a regular scheme $X$, by the Auslander-Buchsbaum theorem. (In fact, in this case $X$ clearly has the resolution property and there always exist locally free sheaves $\mathscr{F}$ and $\mathscr{E}$, and a short exact sequence $0 \rightarrow \mathscr{F} \rightarrow \mathscr{E} \rightarrow \mathscr{I}_{Z} \rightarrow 0$, with $\operatorname{rank} \mathscr{F}=\operatorname{rank} \mathscr{E}-1$; and by the Hilbert-Burch theorem, any Cohen-Macaulay codimension 2 subscheme of $X$ arises in this way.)

Consider the degeneracy $X^{\geq 1+i}\left(\mathscr{I}_{Z}\right)$ for $i \geq 0$ as before (note rank $\mathscr{I}_{Z}=1$ ); then $X^{\geq 1+i}\left(\mathscr{I}_{Z}\right)$ are the loci where the ideal $\mathscr{I}_{Z}$ needs no less than $i+1$ generators. It is known (e.g., [15]) that if $\operatorname{codim} X^{\geq 1+i}\left(\mathscr{I}_{Z}\right) \geq i+1$ for $i \geq 1$, then $\pi: \mathbb{P}\left(\mathscr{I}_{Z}\right)=\mathrm{Bl}_{Z} X \rightarrow X$ is the blowup of $X$ along $Z$ and is irreducible, and $\widetilde{Z}:=\mathbb{P}\left(\mathscr{E} x t^{1}\left(\mathscr{I}_{Z}, \mathscr{O}_{X}\right)\right)$ is the Springer-type desingularization of $Z$. Notice that if $X$ is Gorenstein, then $\widetilde{Z} \simeq \mathbb{P}\left(\mathscr{E} x t^{1}\left(\mathscr{I}_{Z}, \omega_{X}\right)\right)=\mathbb{P}\left(\omega_{Z}\right)$, where $\omega_{X}$ and $\omega_{Z}$ are the dualizing sheaves. Theorem 4.1 states that if either 
(A) $\mathrm{Bl}_{Z} X$ and $\widetilde{Z}$ are smooth and quasi-projective, $\widetilde{Z}$ maps birationally to $Z$ (hence $\widetilde{Z}$ is a resolution of $Z$ ), and $\operatorname{codim} X^{\geq 1+i}\left(\mathscr{I}_{Z}\right) \geq 1+2 i$ for $i \geq 2$ (or equivalently, $\Gamma:=\mathrm{Bl}_{Z} X \times_{X} \widetilde{Z}$ is irreducible and $\left.\operatorname{dim} \Gamma=\operatorname{dim} X-1\right)$, or

(B) $X$ is Cohen-Macaulay and $\operatorname{codim} X^{\geq 1+i}\left(\mathscr{I}_{Z}\right)=i(1+i)$ for $i \geq 1$,

then for any $k \geq 0$, there is an isomorphism of Chow groups

$$
\Gamma^{*} \oplus \pi^{*}: \mathrm{CH}_{k-1}(\widetilde{Z}) \oplus \mathrm{CH}_{k}(X) \stackrel{\sim}{\rightarrow} \mathrm{CH}_{k}\left(\mathrm{Bl}_{Z} X\right) .
$$

\section{Applications}

\subsection{Symmetric powers of curves}

Let $C$ be a smooth projective curve of genus $g \geq 1$ over $\mathbb{C}$, and for $d \in \mathbb{Z}$ denote by $C^{(d)}$ the $d$ th symmetric power of $C$. Then $C^{(d)}$ is smooth projective of dimension $d$, parametrizes effective zero cycles of degree $d$ on $C$. By convention, $C^{(0)}=\{0\}$ is the trivial zero cycle; $C^{(d)}=\emptyset$ for $d<0$. There is an Abel-Jacobi map

$$
A J: C^{(d)} \rightarrow \operatorname{Pic}^{d}(C), \quad A J: D \mapsto \mathscr{O}(D),
$$

where $\operatorname{Pic}^{d}(C)$ is the Picard variety of line bundles of degree $d$ on $C$. The fiber of $A J$ over a point $\mathscr{L}=\mathscr{O}(D) \in X=\operatorname{Pic}^{d}(C)$ is the linear system $|\mathscr{L}|=\mathbb{P}_{\text {sub }}\left(H^{0}(C, \mathscr{L})\right)=$ $\mathbb{P}\left(H^{0}(C, \mathscr{L})^{\vee}\right)$. If $d \geq 2 g-1$, by Riemann-Roch $A J$ is a projective $\mathbb{P}^{d-g}$-bundle over $\operatorname{Pic}^{d}(C)$, which makes the case $0 \leq d \leq 2 g-2$ most interesting. If $g \leq d \leq 2 g-1$, then $A J$ is surjective, with generic fiber $\mathbb{P}^{d-g}$, and the fiber dimension jumps over $W_{d}^{d-g+i}$ for $i \geq 1$, where $W_{d}^{k}$ is the Brill-Noether locus, defined as

$$
W_{d}^{k}:=W_{d}^{k}(C):=\left\{\mathscr{L} \mid \operatorname{dim} H^{0}(C, \mathscr{L}) \geq k+1\right\} \subset \operatorname{Pic}^{d}(C) .
$$

If $0 \leq d \leq g-1$, then $A J$ maps birationally onto the Brill-Noether loci $W_{d}^{0} \subset \operatorname{Pic}^{d}(C)$, which have codimension $g-d$, and the dimension jumps over $W_{d}^{i}$ for $i \geq 1$.

The cases $g-1 \leq d \leq 2 g-2$ and $0 \leq d \leq g-1$ are naturally related by the involution $\mathscr{O}(D) \mapsto \mathscr{O}(K-D)$, which induces a canonical isomorphism $W_{d}^{k} \simeq W_{2 g-2-d}^{g-d+k-1}$. Following Toda [51], from now on we use the following notation: set an integer $n \geq 0$, and set

$$
d=g-1+n, \quad d^{\prime}=2 g-2-d=g-1-n .
$$

(We do not restrict ourselves to $n \leq g-1$, though this is the most interesting case.) Therefore, apart from the usual Abel-Jacobi map, we also have its involution version

$$
A J^{\vee}: C^{\left(d^{\prime}\right)}=C^{(g-1-n)} \rightarrow \operatorname{Pic}^{d}(C), \quad A J^{\vee}: D \mapsto \mathscr{O}\left(K_{C}-D\right) .
$$


The fiber of $A J^{\vee}$ over a point $\mathscr{L} \in \operatorname{Pic}^{d}(C)$ is the linear system $\left|\mathscr{L}^{\vee}\left(K_{C}\right)\right|=$ $\mathbb{P}_{\text {sub }}\left(H^{1}(C, \mathscr{L})^{*}\right)=\mathbb{P}\left(H^{1}(C, \mathscr{L})\right)$. Therefore we have the following fibered diagram:

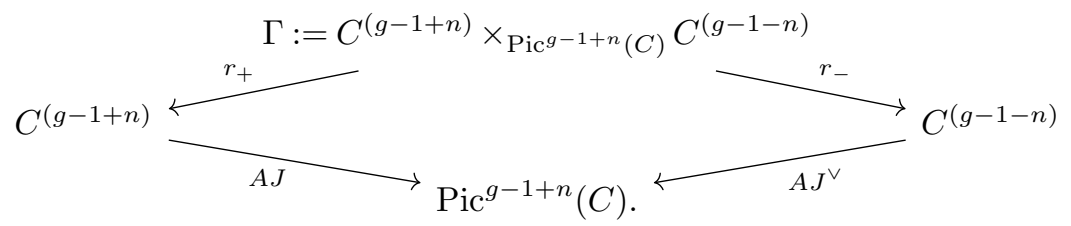

Corollary 5.1. For a smooth projective curve $C$ of genus $g \geq 1$ and integers $n \geq 0, k \geq 0$, there is an isomorphism of integral Chow groups

$$
\mathrm{CH}_{k-n}\left(C^{(g-1-n)}\right) \oplus \bigoplus_{i=0}^{n-1} \mathrm{CH}_{k-(n-1)+i}\left(\mathrm{Pic}^{g-1+n} C\right) \stackrel{\sim}{\rightarrow} \mathrm{CH}_{k}\left(C^{(g-1+n)}\right),
$$

given by $\left(\gamma, \oplus_{i=0}^{n-1} \alpha_{i}\right) \mapsto \beta=\Gamma^{*} \gamma+\sum_{i=0}^{n-1} c_{1}(\mathscr{O}(1))^{i} \cap(A J)^{*} \alpha_{i}$, where $\Gamma^{*}=r_{+*} r_{-}^{*}$ as usual and $\mathscr{O}(1)$ is the line bundle $\mathscr{O}_{\mathbb{P}(\mathscr{G})}(1)$ under the identification $C^{(g-1+n)}=\mathbb{P}(\mathscr{G})$ later. The same map also induces an isomorphism of Chow motives

$$
[\Gamma]^{t} \oplus \bigoplus_{i=0}^{n-1} h^{i} \circ(A J)^{*}: \mathfrak{h}\left(C^{(g-1-n)}\right)(n) \oplus\left(\bigoplus_{i=0}^{n-1} \mathfrak{h}\left(\operatorname{Pic}^{g-1+n}(C)\right)(i)\right) \stackrel{\sim}{\rightarrow} \mathfrak{h}\left(C^{(g-1+n)}\right) .
$$

Notice that $C^{(g-1-n)}=\emptyset$ if $n>g-1$, hence the result is most interesting if $0 \leq n \leq$ $g-1$. To prove the corollary, we show that the foregoing situation fits into the picture of Theorem 4.2 and satisfies condition (A) (if $C$ is not hyperelliptic).

Set $X:=\operatorname{Pic}^{g-1+n}(C)$, and let $D$ be an effective divisor of large degree on $C$. For all $\mathscr{L} \in \operatorname{Pic}(X)$, the exact sequence $\left.0 \rightarrow \mathscr{L} \rightarrow \mathscr{L}(D) \rightarrow \mathscr{L}(D)\right|_{D} \rightarrow 0$ induces an exact sequence

$$
0 \rightarrow H^{0}(C, \mathscr{L}) \rightarrow H^{0}(C, \mathscr{L}(D)) \stackrel{\mu_{D}}{\longrightarrow} H^{0}\left(C,\left.\mathscr{L}(D)\right|_{D}\right) \rightarrow H^{1}(C, \mathscr{L}) \rightarrow 0 .
$$

Globalizing (the dual of) this sequence yields the desired picture: let $\mathscr{L}_{\text {univ }}$ be the universal line bundle of degree $g-1+n$ on $C \times X$, and let $\operatorname{pr}_{C}, \operatorname{pr}_{X}$ be obvious projections. Then

$$
\mathscr{E}:=\left(\operatorname{pr}_{X *}\left(\operatorname{pr}_{C}^{*} \mathscr{O}(D) \otimes \mathscr{L}_{\text {univ }}\right)\right)^{\vee} \quad \text { and } \quad \mathscr{F}:=\left(\operatorname{pr}_{X *}\left(\operatorname{pr}_{C}^{*} \mathscr{O}_{D}(D) \otimes \mathscr{L}_{\text {univ }}\right)\right)^{\vee}
$$

are vector bundles on $X$ of $\operatorname{ranks} \operatorname{deg}(D)+n$ and $\operatorname{deg}(D)$, with a short exact sequence

$$
0 \rightarrow \mathscr{F} \stackrel{\sigma=\mu_{D}^{\vee}}{\longrightarrow} \mathscr{E} \rightarrow \mathscr{G} \rightarrow 0,
$$

where $\mathscr{G}:=\operatorname{Coker}(\sigma)$ is the sheafification of $H^{0}(C, \mathscr{L})^{\vee}$, with homological dimension $\leq 1$ and rank $n$, and $\mathscr{K}:=\mathscr{E} x t^{1}\left(\mathscr{G}, \mathscr{O}_{X}\right)=\operatorname{Coker}\left(\sigma^{\vee}\right)$ is the sheafification of $H^{1}(C, \mathscr{L})$. Therefore

$$
C^{(d)} \equiv C^{(g-1+n)} \simeq \mathbb{P}(\mathscr{G}) \text { and } \quad C^{\left(d^{\prime}\right)} \equiv C^{(g-1-n)} \simeq \mathbb{P}(\mathscr{K}) .
$$


Then the stratification $X_{i}:=X^{\geq n+i+1}(\mathscr{G})$ for $i \geq-1$ of Theorem 4.2 corresponds to Brill-Noether loci as follows (recall $d=g-1+n, d^{\prime}=g-1-n$ ):

$$
X_{i}=W_{d}^{n+i} \simeq W_{d^{\prime}}^{i}
$$

Recall the following facts from [3]:

(1) (Brill-Noether inequality) The expected dimension of $W_{d}^{k}$ is the Brill-Noether number $\rho(g, k, d):=g-(k+1)(g-d+k)$. We have $\operatorname{dim} W_{d}^{k} \geq \rho(g, d, k), W_{d}^{k} \neq \emptyset$ if $\rho(g, k, d) \geq 0$, and $W_{d}^{k}$ is connected if $\rho(g, k, d) \geq 1$.

(2) (Clifford's inequality) For an effective divisor $D$ of degree $d, 1 \leq d \leq 2 g-1$, we have $r(D):=\operatorname{dim} H^{0}(C, \mathscr{O}(D))-1 \leq \frac{1}{2} d$.

(3) (Martens' theorem) Assume $g \geq 3$ and $(d, k) \in\left\{2 \leq d \leq g-1,1 \leq k \leq \frac{d}{2}\right\} \cup$ $\left\{g-1 \leq d \leq 2 g-4, d-g+2 \leq k \leq \frac{\bar{d}}{2}\right\}$. If $C$ is not hyperelliptic, then $\operatorname{dim} W_{d}^{k} \leq$ $d-2 k-1$. If $C$ is hyperelliptic, then $\operatorname{dim} W_{d}^{k}=d-2 k$.

Proof of Corollary 5.1. We proceed by cases.

Uninteresting cases. Note that $\Gamma \neq \emptyset$ if and only if $0 \leq n \leq g-1$; the corollary for the cases $n \geq g$ follows from the projective bundle formula (Theorem 2.4). If $n=g-1$, then $A J^{\vee}: C^{(g-1-n)} \simeq\left\{\left[\omega_{C}\right]\right\} \in \operatorname{Pic}^{2 g-2}(C)$, and $\Gamma=\mathbb{P}_{\text {sub }}\left(H^{0}\left(C, \omega_{C}\right)\right) \simeq \mathbb{P}^{g-1} \subset C^{(2 g-2)}$, and the fibered diagram is a Cayley-trick diagram with $Z=\left\{\left[\omega_{C}\right]\right\}$ a point. Then the results follow from Theorem 3.1. Hence we need only consider the case $0 \leq n \leq g-2$ and $g \geq 2$. If $g=2$, then $n=0, d=d^{\prime}=1, \Gamma \simeq C$, and $\Gamma^{*}: \mathrm{CH}(C) \simeq \mathrm{CH}(C)$ is the isomorphism induced by the hyperelliptic involution on $C$. Hence we may assume from now on $g \geq 3$, $0 \leq n \leq g-2$, and $d=g-1+n \in[g-1,2 g-3]$.

The case $g \geq 3$, C not hyperelliptic. We show that condition (A) is satisfied - that is,

$$
\operatorname{codim}\left(W_{d}^{n} \subset X\right)=n+1, \quad \operatorname{codim}\left(W_{d}^{n+i} \subset X\right) \geq n+2 i+2, \quad \text { for } i \geq 1 .
$$

The first equality always holds, since $C^{\left(d^{\prime}\right)}$ maps birationally onto $W_{d}^{n} \simeq W_{d^{\prime}}^{0}$. For the second inequality, notice that if $d=2 g-3$ and $n=g-2$, then $W_{d}^{n+i}=\emptyset$ if $i \geq 1$ by Clifford's inequality, since $2 n+2 i=2 g-4+2 i>d$ if $i \geq 1$. Hence we may assume $d \in$ $[g-1,2 g-4]$, and Martens' theorem can be applied. Therefore if $C$ is not hyperelliptic, then for any $i \geq 1$,

$$
\operatorname{codim}\left(W_{d}^{n+i} \subset X\right) \geq g-(d-2(n+i)-1)=g-(g-1+n)+2(n+i)+1=n+2 i+2 .
$$

The case $g \geq 3$, C hyperelliptic. Take a disc $D$ in the moduli space $\mathcal{M}_{g}$ intersecting transversely the hyperelliptic locus, with zero point $[C]$, and consider the universal curve $\mathscr{C}$ over $D$. Then the general fiber of $\mathscr{C}$ is nonhyperelliptic, and by the foregoing estimates condition (A) is satisfied by the family $\mathscr{C}$ (with relative Hilbert schemes $\operatorname{Hilb}_{g-1 \pm n}(\mathscr{C} / D$ ) of 0 -dimensional subscheme on the fibers of length $g-1 \pm n$ ) as well as the generic fiber $\mathscr{C}_{\eta}$. Therefore the identities of the maps between Chow groups $\left(\Gamma_{*} \Gamma^{*}=\mathrm{Id}\right.$, the decomposition of Id $=\Gamma^{*} \Gamma_{*}+\sum_{i} \pi_{i}^{*} \pi_{i *}$, etc.) of Theorem 4.1 for $\operatorname{Hilb}_{g-1 \pm n}(\mathscr{C} / D)$ or $\mathscr{C}_{\eta}^{(g-1 \pm n)}$ specialize to the same identities for the central fiber $\mathscr{C}_{0}=C[18, \mathrm{Ch} .10]$ and hence induce the isomorphism of Corollary 5.1 for the hyperelliptic curve $C$. 
Remark 5.2. The isomorphisms of Corollary 5.1 are over the ring $\mathbb{Z}$. If working with rational coefficients, as pointed out to us by the referee, one could also deduce the $\mathbb{Q}$-linear version of Corollary 5.1 from del Baño's works [14, 13]: [13, Proposition 3.7] implies

$$
\mathfrak{h}_{\mathbb{Q}}\left(C^{(n)}\right) \simeq \bigoplus_{n_{0}+n_{1}+n_{2}=n} 1^{\otimes n_{0}} \otimes \lambda^{n_{1}} \mathfrak{h}_{\mathbb{Q}}^{1}(C) \otimes \mathbb{L}^{\otimes n_{2}}=\bigoplus_{n_{0}+n_{1}+n_{2}=n}\left(\lambda^{n_{1}} \mathfrak{h}_{\mathbb{Q}}^{1}(C)\right)\left(n_{2}\right)
$$

where $n_{0}, n_{1}, n_{2}$ are nonnegative integers, $\mathfrak{h}_{\mathbb{Q}}\left(\_\right)=\mathfrak{h}\left(\_\right) \otimes \mathbb{Q}$, and $\lambda^{n}$ is the $\lambda$-structure on the $\mathbb{Q}$-linear pseudoabelian category of effective Chow motives. By [14],

$$
\mathfrak{h}_{\mathbb{Q}}(\operatorname{Jac}(C))=\bigoplus_{k=0}^{2 g} \lambda^{k} \mathfrak{h}_{\mathbb{Q}}^{1}(C)
$$

Combining these two formulae, one obtains the desired result for $\mathfrak{h}_{\mathbb{Q}}$. Using del Baño's works $[14,13]$ in this way, [22, Proposition 1.6] also independently obtains the isomorphism of Chow motives of Corollary 5.1 with rational coefficients.

\subsection{Nested Hilbert schemes of surfaces}

Let $S$ be a smooth surface over $\mathbb{C}$, and for $n \geq 0$, denote $\operatorname{Hilb}_{n}=\operatorname{Hilb}_{n}(S)$ the Hilbert

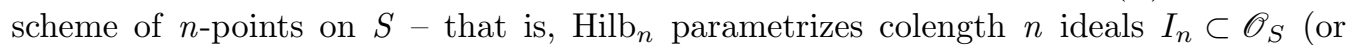
equivalently, length $n 0$-dimension subschemes $\left.\zeta_{n}=V\left(I_{n}\right) \subset S\right)$. Furthermore, define the nested Hilbert scheme by

$$
\operatorname{Hilb}_{n, n+1}=\left\{\left(I_{n+1} \subset I_{n}\right) \mid I_{n} / I_{n+1} \simeq \mathbb{C}(x), \text { for some } x \in S\right\} \subset \operatorname{Hilb}_{n} \times \text { Hilb }_{n+1} .
$$

Then Hilb H,n+1 $_{n, 1}$ parametrizes 0-dimensional subschemes $\eta_{n}=V\left(I_{n}\right) \subset \eta_{n+1}=V\left(I_{n+1}\right) \subset S$ of length $n$ and $n+1$, respectively, such that $\eta_{n+1} / \eta_{n}=\mathbb{C}(x)$ for some $x \in S$. Similarly, one can consider a higher nested Hilbert scheme

$$
\operatorname{Hilb}_{n-1, n, n+1}=\left\{I_{n+1} \subset I_{n} \subset I_{n-1} \mid I_{n} / I_{n+1} \simeq \mathbb{C}(x), I_{n} / I_{n-1} \simeq \mathbb{C}(x) \text {, for some } x \in S\right\} .
$$

Let $X=\operatorname{Hilb}_{n}(S) \times S$, and let $Z_{n} \subset X$ be the universal subscheme. Then $X$ is smooth, and $Z_{n} \subset X$ is a Cohen-Macaulay subscheme of codimension 2.

The following is summarised from Ellingsrud and Strømme [15], Negut [43, 44], and Maulik and Negut [40, Proposition 6.3 \& 6.8]:

\section{Lemma 5.3.}

(1) $\operatorname{Hilb}_{n, n+1}(S)=\mathbb{P}\left(\mathscr{I}_{Z_{n}}\right)=\mathrm{Bl}_{Z_{n}}(X)$ is smooth of dimension $2 n+2$.

(2) $\operatorname{Hilb}_{n-1, n}(S)=\mathbb{P}\left(\mathscr{E} x t^{1}\left(\mathscr{I}_{Z_{n}}, \mathscr{O}_{X}\right)\right)=\mathbb{P}\left(\omega_{Z_{n}}\right)$ is smooth of dimension $2 n$.

(3) $\operatorname{Hilb}_{n-1, n, n+1}(S)=\operatorname{Hilb}_{n-1, n}(S) \times_{X} \operatorname{Hilb}_{n, n+1}(S)$ is smooth of dimension $2 n+1$.

Consider the fibered diagram

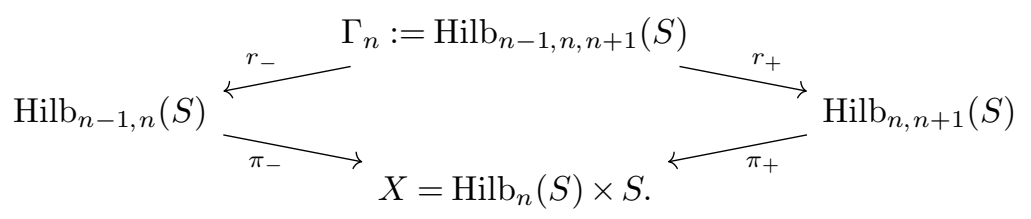




\section{Corollary 5.4.}

(1) For any $k \geq 0$, there is an isomorphism of Chow groups

$$
\mathrm{CH}_{k-1}\left(\operatorname{Hilb}_{n-1, n}(S)\right) \oplus \mathrm{CH}_{k}\left(\operatorname{Hilb}_{n}(S) \times S\right) \stackrel{\sim}{\longrightarrow} \mathrm{CH}_{k}\left(\operatorname{Hilb}_{n, n+1}(S)\right)
$$

given by $(\gamma, \alpha) \mapsto \beta=\Gamma_{n}^{*} \gamma+\pi_{+}^{*} \alpha$. The same map also induces

$$
\left[\Gamma_{n}\right]^{t} \oplus \pi_{+}^{*}: \mathfrak{h}\left(\operatorname{Hilb}_{n-1, n}(S)\right)(1) \oplus \mathfrak{h}\left(\operatorname{Hilb}_{n}(S) \times S\right) \stackrel{\sim}{\rightarrow} \mathfrak{h}\left(\operatorname{Hilb}_{n, n+1}(S)\right) .
$$

(2) Consider the 'zig-zag shape' diagram of length $d \in[0, n]$ :

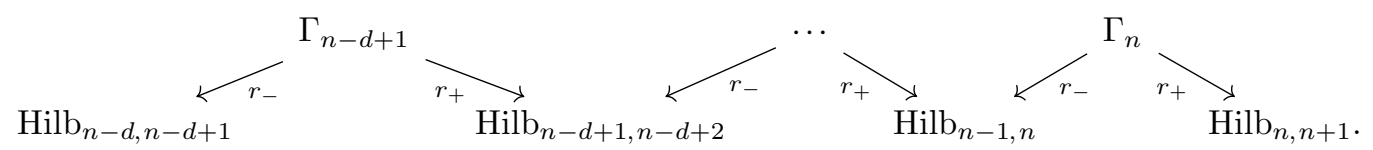

Then it follows from (1) that the following maps are split injective:

$\Gamma_{n}^{*} \Gamma_{n-1}^{*} \cdots \Gamma_{n-d+1}^{*}: \mathrm{CH}_{k-d}\left(\mathrm{Hilb}_{n-d, n-d+1}\right) \hookrightarrow \mathrm{CH}_{k}\left(\mathrm{Hilb}_{n, n+1}\right), \quad$ for $d=1,2, \ldots, n$, $\Gamma_{n}^{*} \Gamma_{n-1}^{*} \cdots \Gamma_{n-d+1}^{*} \pi_{+}^{*}: \mathrm{CH}_{k-d}\left(\operatorname{Hilb}_{n-d} \times S\right) \hookrightarrow \mathrm{CH}_{k}\left(\operatorname{Hilb}_{n, n+1}\right)$, for $d=0,1, \ldots, n$.

It follows similarly for Chow motives. (Note that $\Gamma_{i}^{*}=r_{+*} r_{-}^{*}$ as usual.)

(3) The maps $\Gamma_{n}^{*} \cdots \Gamma_{n-d+1}^{*} \pi_{+}^{*}$ for $d \in[0, n]$ from part (2) induce decompositions

$$
\begin{aligned}
\mathrm{CH}_{k}\left(\operatorname{Hilb}_{n, n+1}(S)\right) & =\mathrm{CH}_{k}\left(\operatorname{Hilb}_{n}(S) \times S\right) \oplus \mathrm{CH}_{k-1}\left(\operatorname{Hilb}_{n-1}(S) \times S\right) \\
& \oplus \cdots \oplus \mathrm{CH}_{k-n+1}(S \times S) \oplus \mathrm{CH}_{k-n}(S), \quad \forall k \geq 0, \\
\mathfrak{h}\left(\operatorname{Hilb}_{n, n+1}(S)\right) & =\mathfrak{h}\left(\operatorname{Hilb}_{n}(S) \times S\right) \oplus \mathfrak{h}\left(\operatorname{Hilb}_{n-1}(S) \times S\right)(1) \\
& \oplus \cdots \oplus \mathfrak{h}(S \times S)(n-1) \oplus \mathfrak{h}(S)(n) .
\end{aligned}
$$

These results are especially interesting in the case when $S$ is a K3 surface [40, 45, 55]. Note that the map $\Gamma_{n} \circ \Gamma_{n-1}$ is also given by the correspondence $\left[\Gamma_{n}\right] *\left[\Gamma_{n-1}\right]=$ $\left[\right.$ Hilb $\left._{n-2, n-1, n, n+1}\right]$. This is because the fiber squares for the fiber product

$$
\operatorname{Hilb}_{n-2, n-1, n, n+1}=\operatorname{Hilb}_{n-2, n-1, n} \times \operatorname{Hilb}_{n-1, n} \operatorname{Hilb}_{n-1, n, n+1}
$$

does not have an excess bundle (see [44, Proposition 2.21]; the result there was shown for stable sheaves, but the same proof works for nested Hilbert schemes).

Proof of Corollary 5.4. Let $\mathscr{G}=\mathscr{I}_{Z}$. It remains to check that condition (A) of Theorem 4.2 is satisfied. In fact, notice that $X^{\geq r+i}(\mathscr{G})=X^{\geq 1+i}\left(\mathscr{I}_{Z_{n}}\right)$ are the loci where $\mathscr{I}_{Z_{n}}$ needs $\geq 1+i$ generators at a point $(I, x)$ - or equivalently,

$$
X^{\geq 1+i}\left(\mathscr{I}_{Z_{n}}\right)=\left\{(I, x) \in \operatorname{Hilb}_{n} \times S \mid \operatorname{dim} I(x) \geq 1+i\right\} .
$$

It follows from [15, proof of Proposition 3.2] that $\operatorname{codim}\left(X^{\geq 1+i} \subset X\right) \geq 2 i$ for all $i \geq 1$. On the other hand, we already know that $\Gamma=\operatorname{Hilb}_{n-1, n, n+1}(S)$ is irreducible and of expected dimension, by Lemma $5.3(3)$; therefore $\operatorname{codim}\left(X^{\geq 1+i} \subset X\right) \geq 1+2 i$ for all $i \geq 2$ and condition (4.1) is satisfied (see Remark 4.2i). Finally, parts (2) and (3) follow from part (1). 
Remark 5.5. De Cataldo and Migliorini established the decompositions of the rational Chow groups of $\operatorname{Hilb}_{n}(S)$ in [11, Corollary 5.1.5] and of $\operatorname{Hilb}_{n, n+1}(S)$ in [12, Theorem 3.3.1]. In view of Remark 5.2, it is reasonable to expect that one could also deduce the $\mathbb{Q}$-linear version of Corollary 5.4 from the decompositions of $\mathrm{CH}\left(\operatorname{Hilb}_{n}(S)\right)_{\mathbb{Q}}$ and $\mathrm{CH}\left(\operatorname{Hilb}_{n, n+1}(S)\right)_{\mathbb{Q}}$ in $[11,12]$.

\subsection{Voisin maps}

Let $Y \subset \mathbb{P}_{\mathbb{C}}^{5}$ be a cubic fourfold not containing any plane, $F(Y)$ be the Fano variety of lines on $Y$ which is a hyperkähler fourfold of type $K 3^{[2]}$, and $Z(Y)$ be the LLSvS eightfold constructed in [36], which is a hyperkähler manifold of type $K 3^{[4]}$. Voisin constructed a rational map $v: F(Y) \times F(Y) \rightarrow Z(Y)$ of degree 6 in [54] using the geometry of $Y$. In [9], Chen showed that the Voisin map $v$ can be resolved by blowing up the incident locus

$$
Z=\left\{\left(L_{1}, L_{2}\right) \in F(Y) \times F(Y) \mid L_{1} \cap L_{2} \neq \emptyset\right\}
$$

using the interpretation [33, 38] of these spaces as moduli of stable objects in the Kuznetsov component $K u(Y)=\left\langle\mathscr{O}_{Y}, \mathscr{O}_{Y}(1), \mathscr{O}_{Y}(2)\right\rangle^{\perp}$ [31], with respect to a Bridgeland stability condition $\sigma$ on $K u(Y)$ constructed in [4].

More precisely, following [9], the Voisin map can be viewed as a family of extensions $v: M_{\sigma}\left(\lambda_{1}\right) \times M_{\sigma}\left(\lambda_{1}+\lambda_{2}\right) \rightarrow M_{\sigma}\left(2 \lambda_{1}+\lambda_{2}\right)$ as follows, where $\lambda_{1}, \lambda_{2} \in \mathcal{K}_{\text {num }}(K u(Y))$ are the natural basis of an $A_{2}$ lattice [1]. By [33, 38], there are identifications of moduli spaces $M_{\sigma}\left(\lambda_{1}\right)=F(Y), M_{\sigma}\left(\lambda_{1}+\lambda_{2}\right)=F(Y)$, and $M_{\sigma}\left(2 \lambda_{1}+\lambda_{2}\right)=Z(Y)$. Let $\mathcal{F}$, $\mathcal{P}$, and $\mathcal{E}$ be the respective pullbacks of the (quasi-)universal objects on $M_{\sigma} \times Y$ to the moduli spaces $M_{\sigma}\left(\lambda_{1}\right), M_{\sigma}\left(\lambda_{1}+\lambda_{2}\right)$, and $M_{\sigma}\left(2 \lambda_{1}+\lambda_{2}\right)$. Then the Voisin map $v$ sends a pair $(F, P) \in M_{\sigma}\left(\lambda_{1}\right) \times M_{\sigma}\left(\lambda_{1}+\lambda_{2}\right)$ which satisfies $\operatorname{dim}_{\operatorname{Ext}^{1}}(F, P)=1$ to the unique class of nontrivial extension of $F$ by $P$.

If we denote $X=F(Y) \times F(Y)$ and let $\mathscr{E} x t_{f}^{i}(\mathscr{F}, \mathcal{P})$ be the sheafification of the group $\operatorname{Ext}^{i}(F, P)$ for the family $f: X \times Y \rightarrow X$, the following are proved in [9]:

(1) $\mathscr{E} x t_{f}^{1}(\mathscr{F}, \mathcal{P})=\mathscr{I}_{Z}$ (where $\mathscr{I}_{Z}$ is the ideal sheaf of $Z \subset X$, and $Z$ is the incident locus $\left\{L_{1} \cap L_{2} \neq \emptyset\right\}$ already defined) has homological dimension 1 , and $Z \subset X=$ $F(Y) \times F(Y)$ is Cohen-Macaulay of codimension 2.

(2) The degeneracy loci of $\mathscr{E} x t_{f}^{1}(\mathscr{F}, \mathcal{P})=\mathscr{I}_{Z}$ over $X$ are given by $\left(X=X^{\geq 1}\left(\mathscr{I}_{Z}\right)\right.$ and $)$

$$
\begin{aligned}
Z & =X^{\geq 2}\left(\mathscr{I}_{Z}\right)=\left\{(F, P) \mid \operatorname{dim} \operatorname{Ext}^{1}(F, P) \geq 2\right\}, \\
\Delta_{2} & =X^{\geq 3}\left(\mathscr{I}_{Z}\right)=\left\{(F, P) \mid \operatorname{dim}_{E_{x t}}{ }^{1}(F, P) \geq 3\right\},
\end{aligned}
$$

and $X^{\geq 1+i}\left(\mathscr{I}_{Z}\right)=\emptyset$ for $i \geq 3$. Here $\Delta_{2} \subset F(Y) \times F(Y)$ is the type II locus $\left\{L \in \Delta \simeq F(Y) \mid \mathscr{N}_{L / Y} \simeq \mathscr{O}(1)^{\oplus 2} \oplus \mathscr{O}(-1)\right\}$, which is an algebraic surface [54].

(3) $\mathscr{E} x t^{1}\left(\mathscr{I}_{Z}, \mathscr{O}_{X}\right)=\mathscr{E} x t_{f}^{2}(\mathcal{P}, \mathcal{F})=\omega_{Z}$, where $\omega_{Z}$ is the dualizing sheaf of $Z$.

(4) The blowup $\pi: \mathbb{P}\left(\mathscr{I}_{Z}\right)=\mathrm{Bl}_{Z}(F(Y) \times F(Y)) \rightarrow F(Y) \times F(Y)$ resolves the Voisin map $v$, and if $Y$ is very general (i.e., $\mathcal{K}_{\text {num }}(K u(Y))=A_{2}$ ), then the resolved Voisin map 
$\widetilde{v}: \mathrm{Bl}_{Z}(F(Y) \times F(Y)) \rightarrow Z(Y)$ is (the projection of) a relative Quot-scheme

$$
\mathrm{Bl}_{Z}(F(Y) \times F(Y))=\operatorname{Quot}_{K u(Y) / Z(Y)}\left(\mathcal{E}, \lambda_{1}+\lambda_{2}\right)
$$

of stable quotients of $\mathcal{E}$ inside $\mathcal{A} \subset K u(Y)$ over $Z(Y)$, where $\mathcal{A}$ is the heart of $\sigma$.

Therefore the sheaf $\mathscr{I}_{Z}$ satisfies condition (B) of Theorem 4.2. If we consider

$$
\pi^{\prime}: \widetilde{Z}:=\mathbb{P}_{X}\left(\mathscr{E} x t_{f}^{2}(\mathcal{P}, \mathcal{F})\right)=\mathbb{P}_{Z}\left(\omega_{Z}\right) \rightarrow X
$$

which is a small (partial) resolution of the incidence locus $Z$, then the projection $\widetilde{Z} \rightarrow Z \subset$ $X$ is an isomorphism over $Z \backslash \Delta_{2}$ and a $\mathbb{P}^{1}$-bundle over $\Delta_{2}$. Therefore we have a diagram

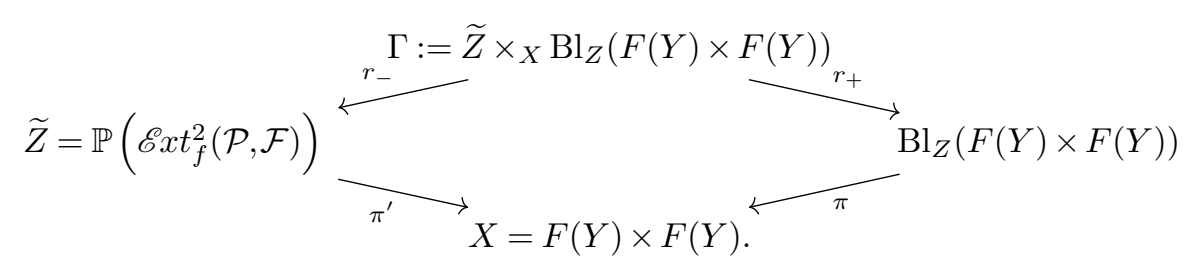

Corollary 5.6. For any $k \geq 0$, there is an isomorphism of Chow groups

$$
\Gamma^{*} \oplus \pi^{*}: \mathrm{CH}_{k-1}(\widetilde{Z}) \oplus \mathrm{CH}_{k}(F(Y) \times F(Y)) \stackrel{\sim}{\longrightarrow} \mathrm{CH}_{k}\left(\mathrm{Bl}_{Z}(F(Y) \times F(Y))\right),
$$

where $\Gamma^{*}=r_{+*} r_{-}^{*}$ as usual. If $\widetilde{Z}$ and $\mathrm{Bl}_{Z}(F(Y) \times F(Y))$ are smooth, then the same map induces an isomorphism of Chow motives

$$
[\Gamma]^{t} \oplus \pi^{*}: \mathfrak{h}(\widetilde{Z})(1) \oplus \mathfrak{h}\left((F(Y) \times F(Y)) \stackrel{\sim}{\rightarrow} \mathfrak{h}\left(\mathrm{Bl}_{Z}(F(Y) \times F(Y))\right) .\right.
$$

Note that from [25], it follows that there is a semiorthogonal decomposition

$$
D\left(\mathrm{Bl}_{Z}(F(Y) \times F(Y))\right)=\langle D(F(Y) \times F(Y)), D(\widetilde{Z})\rangle,
$$

and therefore $\widetilde{Z}$ is smooth if and only if $\mathrm{Bl}_{Z}(F(Y) \times F(Y))$ is. If this is the case, ${ }^{2}$ since the resolution $\widetilde{Z} \rightarrow Z$ is IH-small, through taking the Betti cohomology realization of the Chow motives, this map induces isomorphisms of Hodge structures

$$
\begin{aligned}
H^{n}\left(\mathrm{Bl}_{Z}(F(Y) \times F(Y)), \mathbb{Q}\right) & \simeq H^{n}(F(Y) \times F(Y), \mathbb{Q}) \oplus H^{n-2}(\widetilde{Z}, \mathbb{Q}) \\
& \simeq H^{n}(F(Y) \times F(Y), \mathbb{Q}) \oplus \operatorname{IH}^{n-2}(Z, \mathbb{Q})
\end{aligned}
$$

for any $n \geq 0$, where $\mathrm{IH}$ is the intersection cohomology.

\subsection{Further speculations}

(1) Let $\sigma: \mathscr{F} \rightarrow \mathscr{E}$ be a map between vector bundles over a Cohen-Macaulay scheme $S$; then there is a section map $s_{\sigma}: S \rightarrow|\operatorname{Hom}(\mathscr{E}, \mathscr{F})|$. Condition (B) of Theorem 4.2

\footnotetext{
${ }^{2}$ In fact, one can show $\widetilde{Z}$ is smooth if $\Delta_{2}$ is a smooth surface (see, e.g., [25, Lemma B3]); Amerik [2] shows that $\Delta_{2}$ is smooth for a general $Y$. On the other hand, if $Y$ is very general, Chen's interpretation [9] of $\widetilde{Z}$ as a $Q u o t$-scheme over $X$ shows that $\mathrm{Bl}_{Z} X$ is smooth.
} 
always holds over $|\operatorname{Hom}(\mathscr{E}, \mathscr{F})|$. Assume that a suitable relative Chow theory $\mathrm{CH}(X \rightarrow S)$ has a well-behaved Tor-independent base-change theory, similar to the base-change theory for derived categories [32]. Then one can pull back along the section map $\sigma$ and obtain a projectivization formula for $S$ under a much weaker condition. The candidate theories we have in mind are Fulton's bivariant intersection theory [18, Chapter 17], the theory of pure Chow motives over a base $S$ [10], and the theory of higher Chow groups over a base $S$ [37, Chapter II].

(2) This work is inspired by its counterpart in derived categories [25], where the projectivization formula was proved using the techniques developed in [26, 30 49]. It is interesting whether or not one can 'decategorify' other interesting semiorthogonal decompositions obtained by these techniques. Examples include various cases of homological projective duality and flops [26, 25, 30, 49]. Note that usually, results of derived categories imply only ungraded results for rational Chow groups and motives; but see [7], where essential graded information of Chow groups is recovered from derived categories.

(3) The projectivization formula for derived categories is closely related to the wallcrossing and d-critical flips studied by Toda [50, 51]. It would be interesting to extend the results of this paper to the cases of Donaldson-Thomas-type moduli spaces considered there.

(4) The projectivization formula considered in this paper fits into a broad framework of the study of Quot-schemes of locally free quotients [23, 24].

(5) Since the resolution $\mathbb{P}\left(\mathscr{E} x t^{1}\left(\mathscr{G}, \mathscr{O}_{X}\right)\right) \rightarrow X_{\mathrm{sg}}(\mathscr{G})$ is usually IH-small, it is reasonable to expect that one may replace $\mathrm{CH}\left(\mathbb{P}\left(\mathscr{E} x t^{1}\left(\mathscr{G}_{,} \mathscr{O}_{X}\right)\right)\right)$ by the intersection Chow group [10] of $X_{\mathrm{sg}}(\mathscr{G})$.

(6) The projectivization formula of Chow groups should hold for Deligne-Mumford stacks, with $\mathrm{CH}$ replaced by $\mathrm{CH}_{\mathbb{Q}}$. It would also be interesting to study the ring structure of $\mathrm{CH}(\mathbb{P}(\mathscr{G}))$ in the case when $X$ and $\mathbb{P}(\mathscr{G})$ are smooth.

Acknowledgments. The author would like to thank Arend Bayer for many helpful discussions and Dougla Davis for helpful conversations. The author especially thanks Huachen Chen for bringing his attention to this problem and for many helpful discussions on Voisin maps and his work [9]. This project started during a workshop at Liverpool; the author thanks the organizers, Alice Rizzardo and Theo Raedschelders, for hospitality. The author also thanks the referee for the careful reading and many helpful suggestions, which greatly improved the exposition of the paper. This work is supported by the Engineering and Physical Sciences Research Council (EPSRC) [EP/R034826/1].

Competing Interest. None.

\section{References}

[1] N. Addington and R. Thomas, Hodge theory and derived categories of cubic fourfolds, Duke Math. J. 163(10) (2014), 1885-1927. 
[2] E. Amerik, A computation of invariants of a rational self-map, Ann. Fac. Sci. Toulouse Math. (6) 18(3) (2009), 481-493.

[3] E. Arbarello, M. Cornalba, P. Griffiths and J. Harris, Geometry of Algebraic Curves. Volume I, Grundlehren der mathematischen Wissenschaften, 267 (SpringerVerlag, New York, 1985).

[4] A. Bayer, M. Lahoz, E. Macrì and P. Stellari, Stability Conditions on Kuznetsov Components, Annales Scientifiques de l'École Normale Supérieure (Elsevier, Amsterdam, 2021). arXiv:1703.10839.

[5] P. Belmans and A. Krug, 'Derived categories of (nested) Hilbert schemes', Preprint, 2019, arXiv:1909.04321.

[6] M. Bernardara, M. Bolognesi and D. Faenzi, Homological projective duality for determinantal varieties, Adv. Math. 296 (2016), 181-209.

[7] M. Bernardara and G. Tabuada, Chow groups of intersections of quadrics via homological projective duality and (Jacobians of) non-commutative motives, Izv. Math. 80(3) (2016), 463-480.

[8] A. Bondal And D. Orlov, 'Semiorthogonal decomposition for algebraic varieties', Preprint, 1995, arXiv:9506012.

[9] H. Chen, 'The Voisin map via families of extensions, Preprint, 2018, arXiv:1806.05771.

[10] A. Corti and M. Hanamura, Motivic decomposition and intersection Chow groups. I, Duke Math. J. 103 (2000), 459-522.

[11] M. A. A. de Cataldo And L. Migliorini, The Chow groups and the motive of the Hilbert scheme of points on a surface, J. Algebra 251(2) (2002), 824-848.

[12] M. A. A. de Cataldo And L. Migliorini, The Chow motive of semismall resolutions, Math. Res. Lett. 11 (2004), 151-170.

[13] S. Del BAÑo, On the Chow motive of some moduli spaces, J. Reine Angew. Math. 532 (2001), 105-132.

[14] S. DEL BAÑO, 'On motives and moduli spaces of stable vector bundles over a curve', Thesis, 1998, Universitat Politécnica de Catalunya (UPC).

[15] G. Ellingsrud and S. Strømme, An intersection number for the punctual Hilbert scheme of a surface, Trans. Amer. Math. Soc. 350(6) (1998), 2547-2552.

[16] B. Fu AND C.-L. WANG, Motivic and quantum invariance under stratified Mukai flops, J. Differential Geom. 80(2) (2008), 261-280.

[17] L. Fu, V. Hoskins and S. P. Lehalleur, 'Motives of moduli spaces of rank 3 vector bundles and Higgs bundles on a curve', Preprint, 2021, arXiv:2102.07546.

[18] W. Fulton, Intersection Theory (Springer Science \& Business Media, Berlin, Heidelberg, New York, Tokyo, 1984).

[19] W. Fulton and P. Pragacz, Schubert Varieties and Degeneracy Loci (Springer, Berlin, Heidelberg, 1998).

[20] I. M. Gelfand, M. Kapranov And A. Zelevinsky, Discriminants, Resultants, and Multidimensional Determinants (Springer Science \& Business Media, New York, 2008).

[21] M. Golubitsky and V. Guillemin, Stable Mappings and Their Singularities. Vol. 14 (Springer Science \& Business Media, New York, 2012).

[22] T. Gómez And K.-S. LeE, 'Motivic decompositions of moduli spaces of vector bundles on curves', Preprint, 2020, arXiv:2007.06067.

[23] Q. JiAng, 'On the Chow theory of Quot schemes of locally free quotients', Preprint, 2020, arXiv:2010.10734.

[24] Q. JiAng, 'Derived categories of Quot schemes of locally free quotients, I', Preprint, 2021, arXiv:2107.09193.

[25] Q. Jiang And N. Leung, 'Derived category of projectivization and flops', Preprint, 2018, arXiv:1811.12525. 
[26] Q. JiAng, N. LeUng And Y. XIE, Categorical Plücker formula and homological projective duality, J. Eur. Math. Soc. (JEMS) 23(6) (2021), 1859-1898.

[27] B. Kahn, J. P. Murre And C. Pedrini, On the transcendental part of the motive of a surface, in Algebraic Cycles and Motives. Vol. 2, London Mathematical Society Lecture Note Series, 344, pp. 143-202 (Cambridge University Press, Cambridge, UK, 2007).

[28] Y.-H. Kiem, I.-K. Kim, H. LeE And K.-S. LeE, All complete intersection varieties are Fano visitors, Adv. Math. 311 (2017), 649-661.

[29] N. Koseki And Y. TodA, 'Derived categories of Thaddeus pair moduli spaces via d-critical flips', Preprint, 2019, arXiv:1904.04949.

[30] A. Kuznetsov, Homological projective duality, Publ. Math. Inst. Hautes Etudes Sci. 105(1) (2007), 157-220.

[31] A. Kuznetsov, Derived categories of cubic fourfolds, in Cohomological and Geometric Approaches to Rationality Problems, pp. 219-243 (Springer, Birkhäuser Boston, 2010).

[32] A. Kuznetsov, Base change for semiorthogonal decompositions, Compos. Math. 147 (2011), 852-876.

[33] M. Lahoz, M. Lehn, E. Macrì And P. Stellari, Generalized twisted cubics on a cubic fourfold as a moduli space of stable objects, J. Math. Pures Appl. (9) 114 (2018), 85-117.

[34] R. Lazarsfeld, Positivity in Algebraic Geometry II, Ergebnisse der Mathematik und ihrer Grenzgebiete. 3. Folge / A Series of Modern Surveys in Mathematics 49 (Springer Science \& Business, Berlin, Heidelberg, 2004).

[35] Y.-P. LeE, H.-W. Lin And C.-L. WANG, Flops, motives, and invariance of quantum rings, Ann. of Math. (2) $\mathbf{1 7 2}$ (2010), 243-290.

[36] C. Lehn, M. Lehn, C. Sorger and D. Van Straten, Twisted cubics on cubic fourfolds, J. Reine Angew. Math. 2017(731) 2017, 87-128.

[37] M. Levine, Mixed Motives, Mathematical Surveys and Monographs. 57 (American Mathematical Society, Providence, RI, 1998).

[38] C. Li, L. Pertusi and X. Zhao, 'Twisted cubics on cubic fourfolds and stability conditions', Preprint, 2018, arXiv:1802.01134.

[39] J. I. MAnin, Correspondences, motives and monoidal transformations, Math. USSR Sb. 6(4) (1968), 439-470.

[40] D. Maulik And A. Neguty, Lehn's formula in Chow and conjectures of Beauville and Voisin, J. Inst. Math. Jussieu: 2020-08-03, p.1-39, arXiv:1904.05262.

[41] J. P. Murre, On the motive of an algebraic surface', J. Reine Angew. Math. 409 (1990), 190-204.

[42] J. P. MurRe, On a conjectural filtration on the Chow groups of an algebraic variety. I. The general conjectures and some examples, Indag. Math. (N.S.) 4(2) (1993), 177-188.

[43] A. Neguţ, ' $W$-algebras associated to surfaces', Preprint, 2017, arXiv:1710.03217.

[44] A. Neguţ, 'Hecke correspondences for smooth moduli spaces of sheaves, Preprint, 2018, arXiv:1804.03645.

[45] G. OBerdieck, 'A Lie algebra action on the Chow ring of the Hilbert scheme of points of a K3 surface', Preprint, 2019, arXiv:1908.08830.

[46] D. OrLov, Triangulated categories of singularities, and equivalences between LandauGinzburg models, Mat. Sb. 197(12) (2006), 117-132.

[47] U. RIEß, On the Chow ring of birational irreducible symplectic varieties, Manuscripta Math. 145(3-4) (2014), 473-501.

[48] M. Thaddeus, Stable pairs, linear systems and the Verlinde formula, Invent. Math. 117(2) (1994), 317-353.

[49] R. P. Thomas, Notes on homological projective duality, in Algebraic Geometry: Salt Lake City 2015, Proceedings of Symposia in Pure Mathematics, 97, pp. 585-609 (American Mathematical Society, Providence, RI, 2018). 
[50] Y. TodA, 'Birational geometry for d-critical loci and wall-crossing in Calabi-Yau 3-folds', Preprint, 2018, arXiv:1805.00182.

[51] Y. Toda, 'Semiorthogonal decompositions of stable pair moduli spaces via d-critical flips', Preprint, 2018, arXiv:1805.00183.

[52] C. Vial, Algebraic cycles and fibrations, Doc. Math. 18 (2013), 1521-1553.

[53] C. Vial, Chow-Kuenneth decomposition for 3- and 4-folds fibred by varieties with trivial Chow group of zero-cycles, J. Algebraic Geom. 24(1) (2015), 51-80.

[54] C. Voisin, Remarks and questions on coisotropic subvarieties and 0-cycles of hyper-Kähler varieties, in K3 Surfaces and Their Moduli, pp. 365-399 (Springer, Springer, Berlin, Heidelberg, 2016).

[55] Q. YIN, Finite-dimensionality and cycles on powers of K3 surfaces, Comment. Math. Helv. 90(2) (2015), 503-511. 